\title{
Perturbazioni geomagnetiche, aurorali, ionosferiche e dei raggi cosmici: interdipendenze e relazioni con l'attività solare
}

\author{
F. MaRiani $(*)$-F. MoLina $(* *)$
}

\section{PARTE I}

\section{I'AURORA POLARE (***)}

\subsection{Generalità e classificazione dei tipi aurorali.}

L'aurora polare presenta grande varietà di aspetti nei quali, tuttavia, si ravvisano dei caratteri generali che permettono una veduta abbastanza organica della morfologia.

In questi ultimi dieci anni, alle osservazioni visuali si è aggiunto il metodo radioelettrico, basato sullo studio dei radioechi riflessi dai banchi di ionizzazione presenti ad altezze di circa $100 \mathrm{~km}$, denominati nel loro complesso radioaurora (ionized aurora). Mentre ai risultati delle misurazioni sulla radioaurora non è ancora possibile dare una precisa, definitiva classificazione, per quelli relativi alle aurore visuali è possibile invece dare un quadro abbastanza completo e organizzato.

A scopo di chiarezza, anche in considerazione della rarità del fenomeno in Italia, riportiamo la classificazione dei principali tipi di aurora visuale, secondo le convenzioni adottate dalla Unione Geofisica e Geodetica Internazionale $\left({ }^{1}\right)$, recentemente perfezionate $\left({ }^{2}\right)$.

I fenomeni aurorali si raggruppano in tre categorie principali:

a) Forme a struttura non raggiata

b) Forme a struttura raggiata

c) Aurore fiammeggianti (flaming aurorae).

(*) Istituto Nazionale di Geofisica, Roma - Istituto di Fisica dell'Università, Perugia.

(**) Istituto Nazionale di Geofisica, Roma.

$(* * *)$ Il primo capitolo di questo lavoro è pubblicato in Ann. Geof. 12, 297 (1959). 
Ovviamente i limiti tra le diverse categorie non sono netti, ma più o meno graduali.

2.1.1. Forme a struttura non raggiata. - a) Archi omogenei (homogeneous arcs, HA). - Consistono (Tav. I) in una luminosità diffusa in forma di arco di cerchio, che puó occupare tutta la volta celeste e che, allorchè è vicina all'orizzonte, appare da questo separata mediante un segmento oscuro. La luminosità in ogni caso è più diffusa nella parte alta e più netta nella parte bassa. Quando un arco si trova in prossimità dello zenith, esso puó dividersi in archi paralleli più stretti, o anche assumere struttura fibrosa irregolare.

In generale, specialmente nelle regioni polari, gli archi sono disposti in modo tale che il punto di altezza zenitale maggiore è nella direzione del meridiano geomagnetico. Il colore è di solito da grigio a giallo-verde e solo talvolta rosso. L'arco omogeneo può persistere per diverse ore; spesso cambia lentamente la sua posizione spostandosi verso latitudini geomagnetiche più basse. Può tramutarsi in un arco a struttura raggiata nello spazio di pochi minuti.

b) Bande omogenee (homogeneous bands, HB). - Sono zone in forma di nastro (Tav. II e III), che possono essere rapidamente variabili e il cui bordo inferiore è spesso assai irregolare. Talvolta hanno andamento semicircolare o ellissoidale, oppure mostrano rientranze cosi da apparire come una specie di enorme sipario. Lo spessore può variare entro limiti assai larghi. La luminosità puó anche essere assai intensa. Spesso le bande omogenee si trasformano in bande a struttura raggiata.

c) Archi pulsanti (pulsating arcs, PA). - Sono archi o parti di arco che appaiono e scompaiono ritmicamente con periodo da pochi secondi a un minuto e più; compaiono di frequente dopo una o due notti di intense ed estese aurore. Spesso subiscono lenti spostamenti.

d) Superfici luminose diffuse (diffuse surfaces, IDS). - Consistono in una specie di velo luminoso privo di contorni ben definiti, che occupa quasi tutto il campo visibile; oppure, in altri casi, in zone luminose più isolate e dall'apparenza simile a quella delle nuvole. Se ne osservano due tipi, l'uno di colore verdastro che compare durante periodi di grande attività solare, l'altro di colore da grigio a giallo-verde.

e) Debole luminosità in vicinanza dell'orizzonte (glow, G). - E una debole luminosità bianca o rossastra, somigliante alla luce del crepuscolo, che appare in prossimità dell'orizzonte. Spesso è la parte supe- 


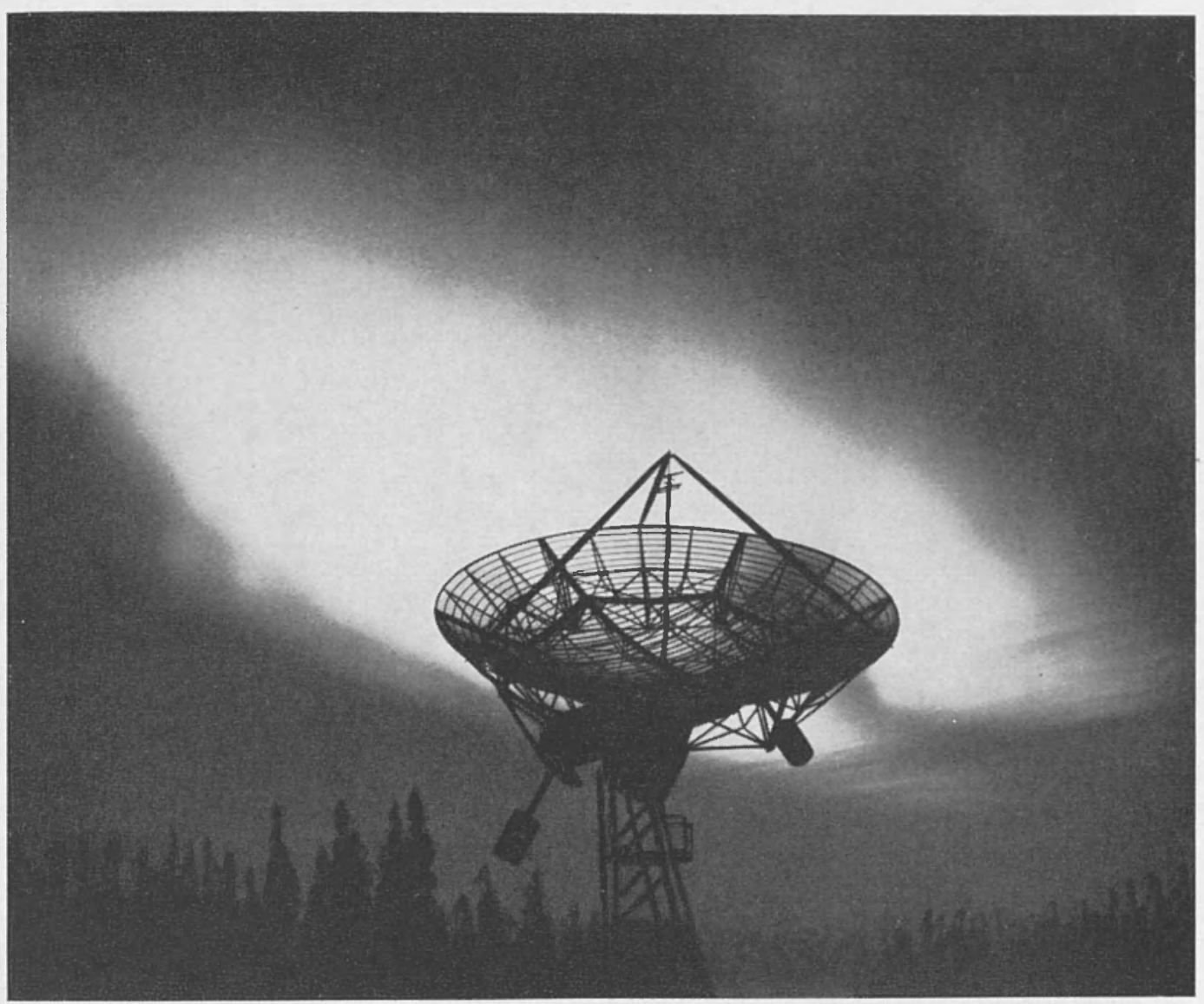

Areo omogeneo (per cortesia del dr. V. P. Hessler del Geophysical Institute University of dlaska. ('ollege). 


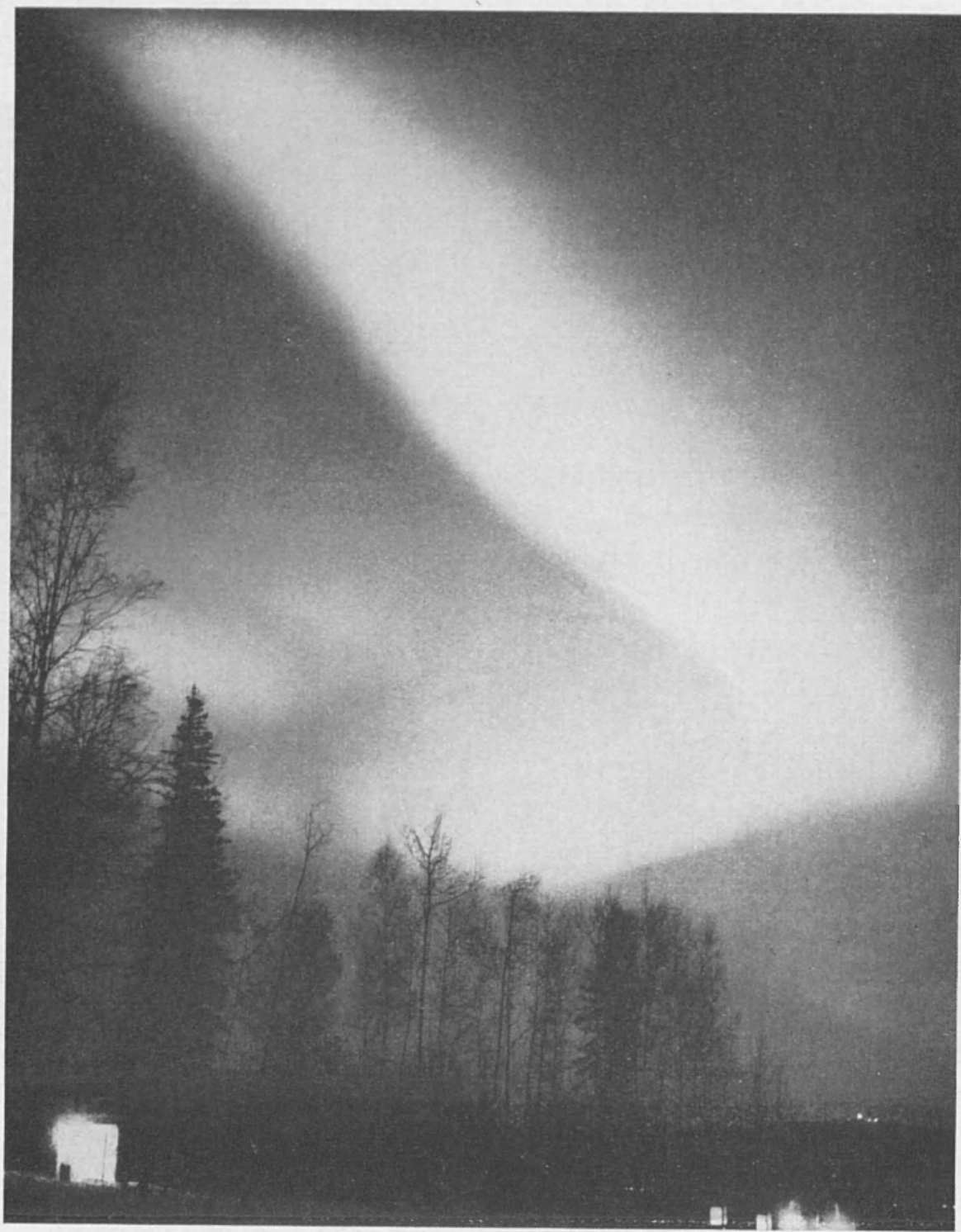

Banda omogenea (per cortesia del dr. V. P. Hessler del Geophysical Institute University of Alaska, College). 


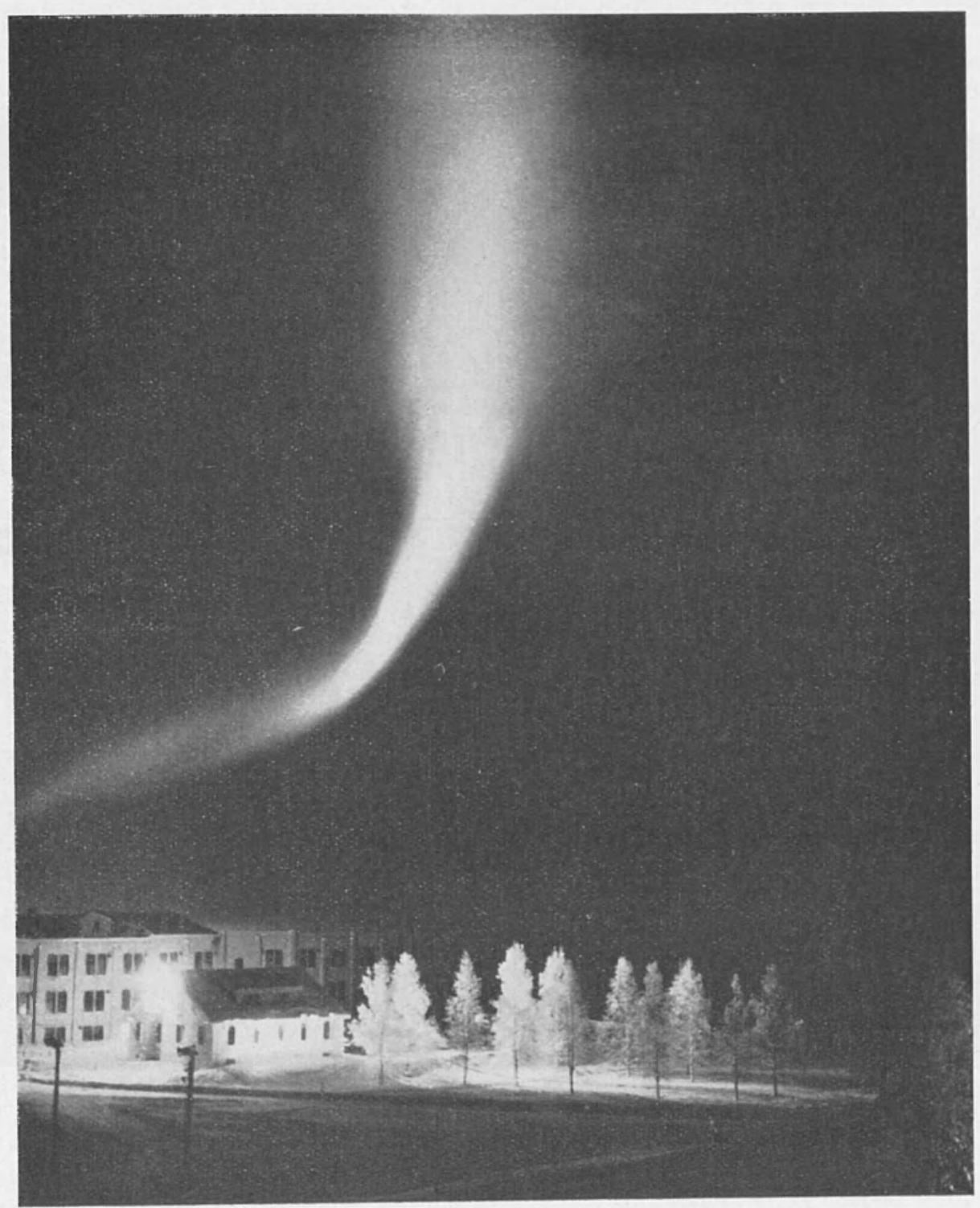

Altro esempiödi handa omogenea (per cortesia del dr. V. P. Hessler del Geoplysical Institute Lniversity of Alaslia, (ollege). 


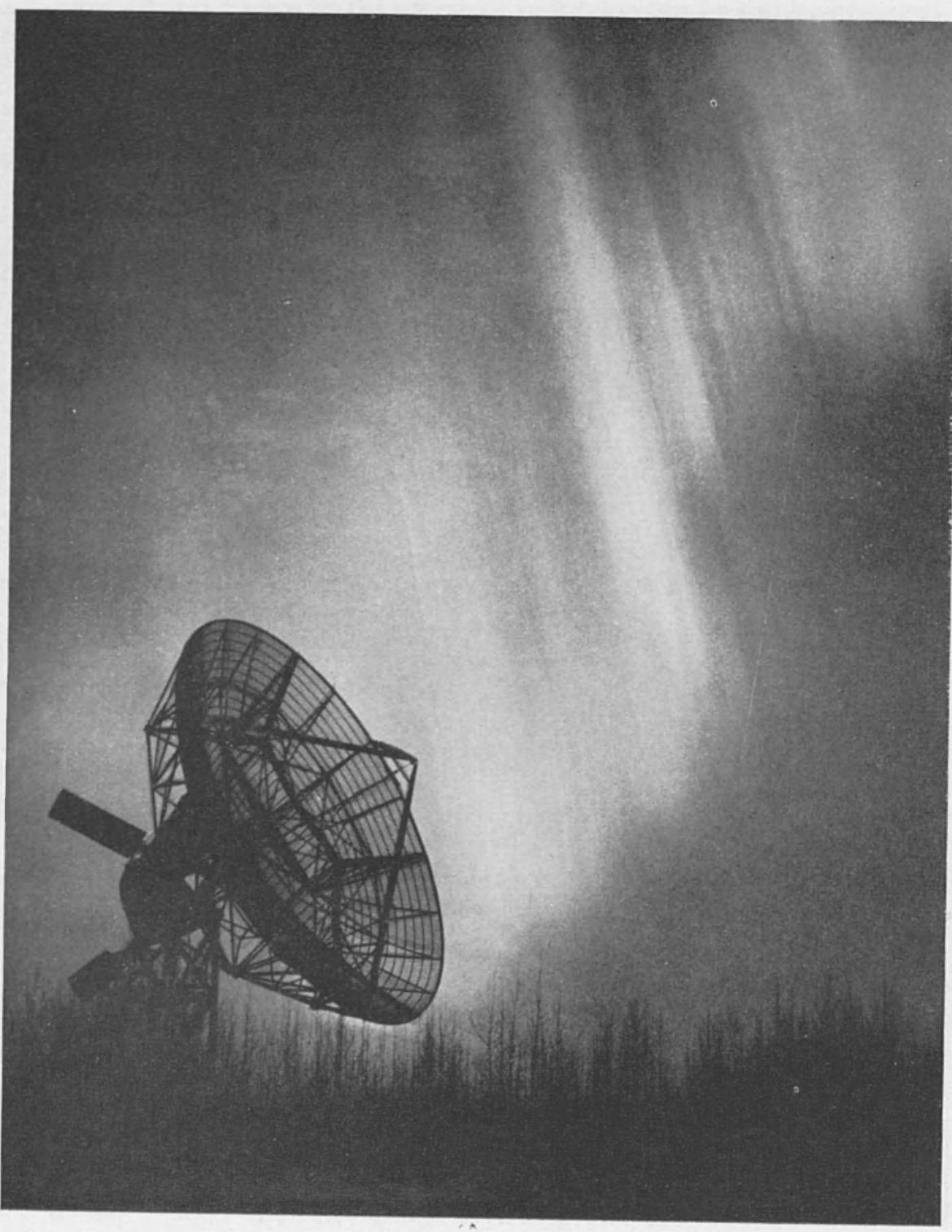

Fascio di raggi in un arco raggiato (per cortesia del dr. V. P. Hessler del Geophysical Institute University of Alaska, College). 


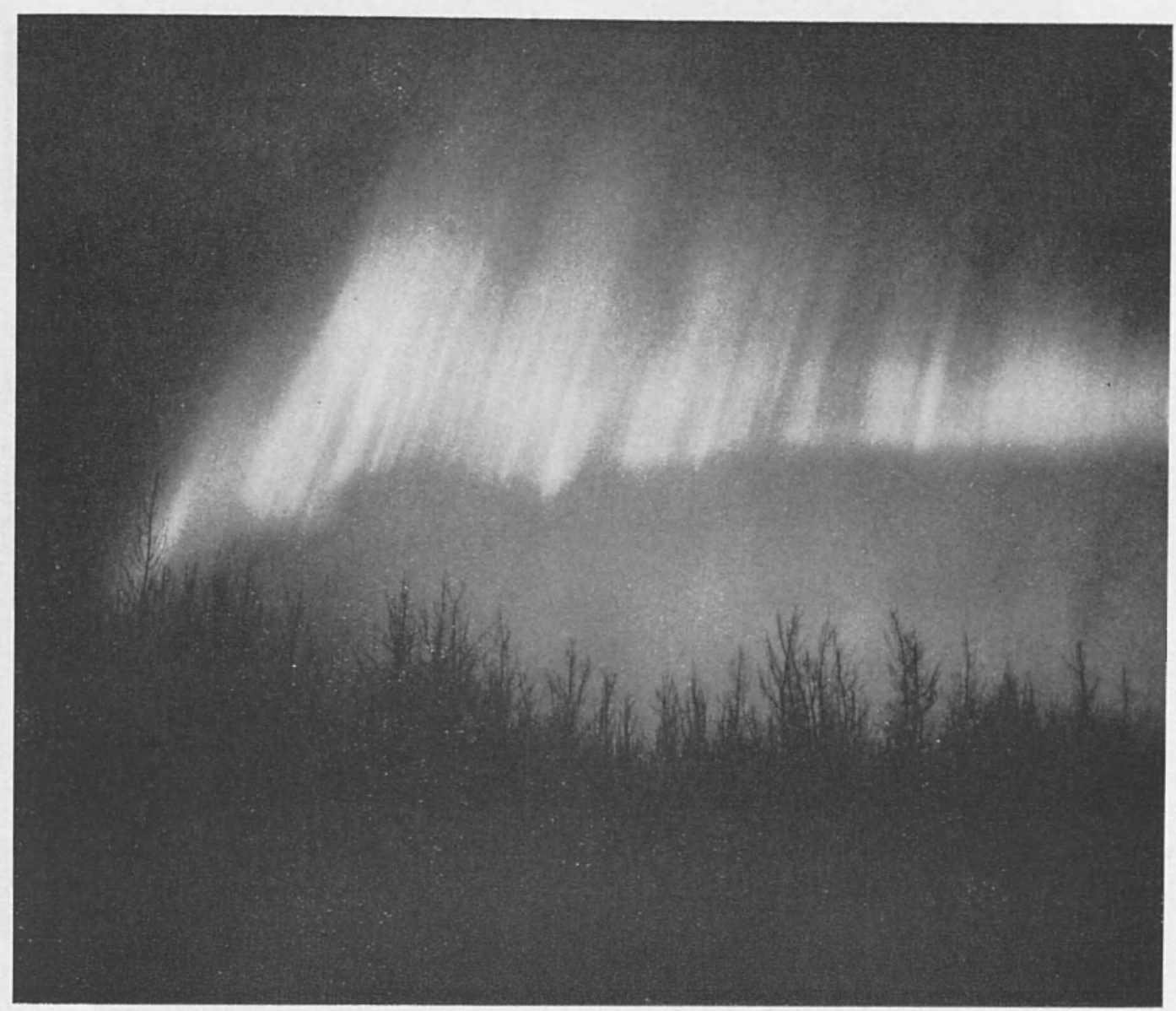

Arco raggiato „(per cortesia del dr. V. P. Hessler del Geopliysical Institute University of Alaska, College). 
Tav. VI

F. MARIANI-F. MOLINA

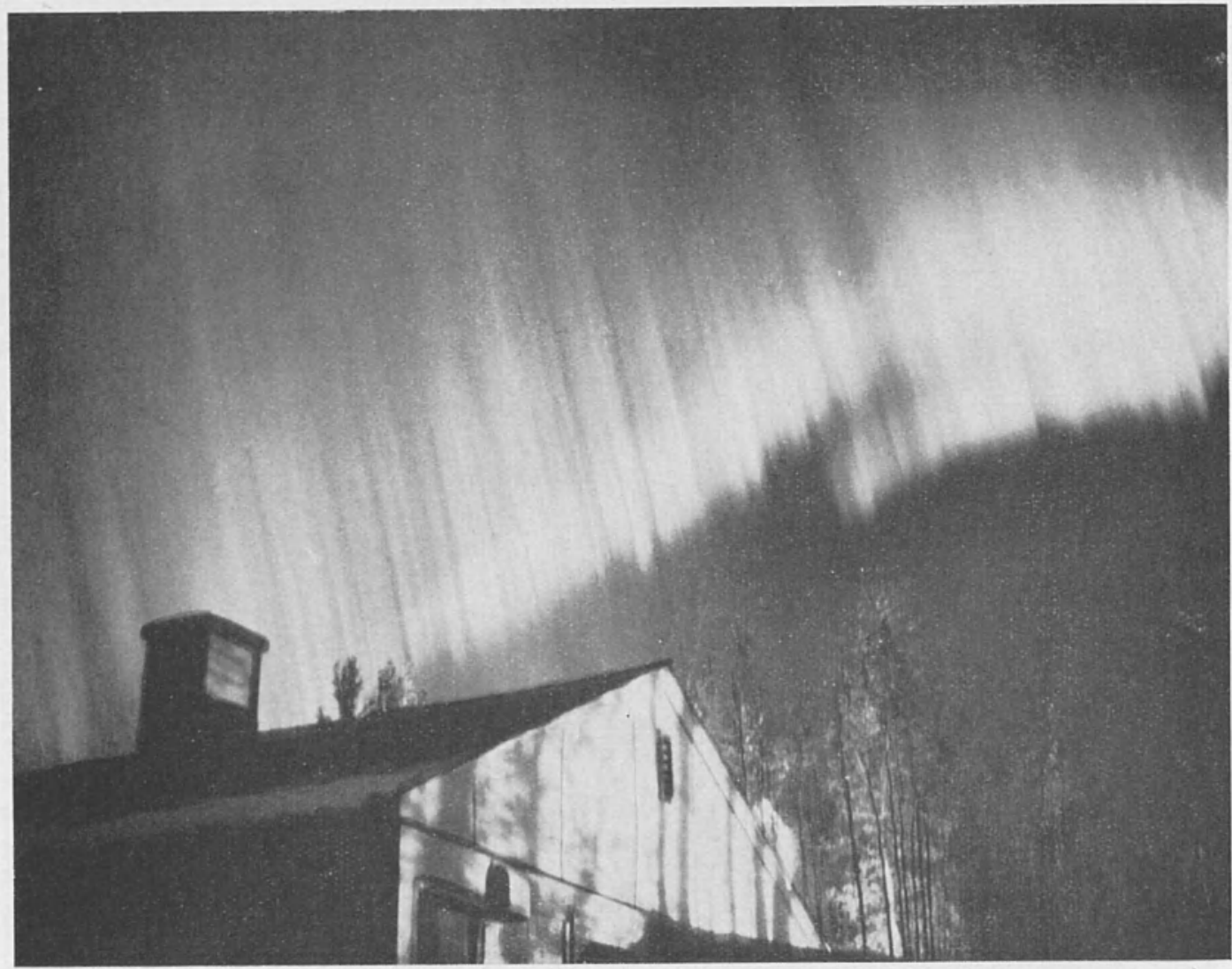

Banda raggiata (per cortesia del dr. V. P. Hessler del Geophysical Institute University of Alaska, College). 


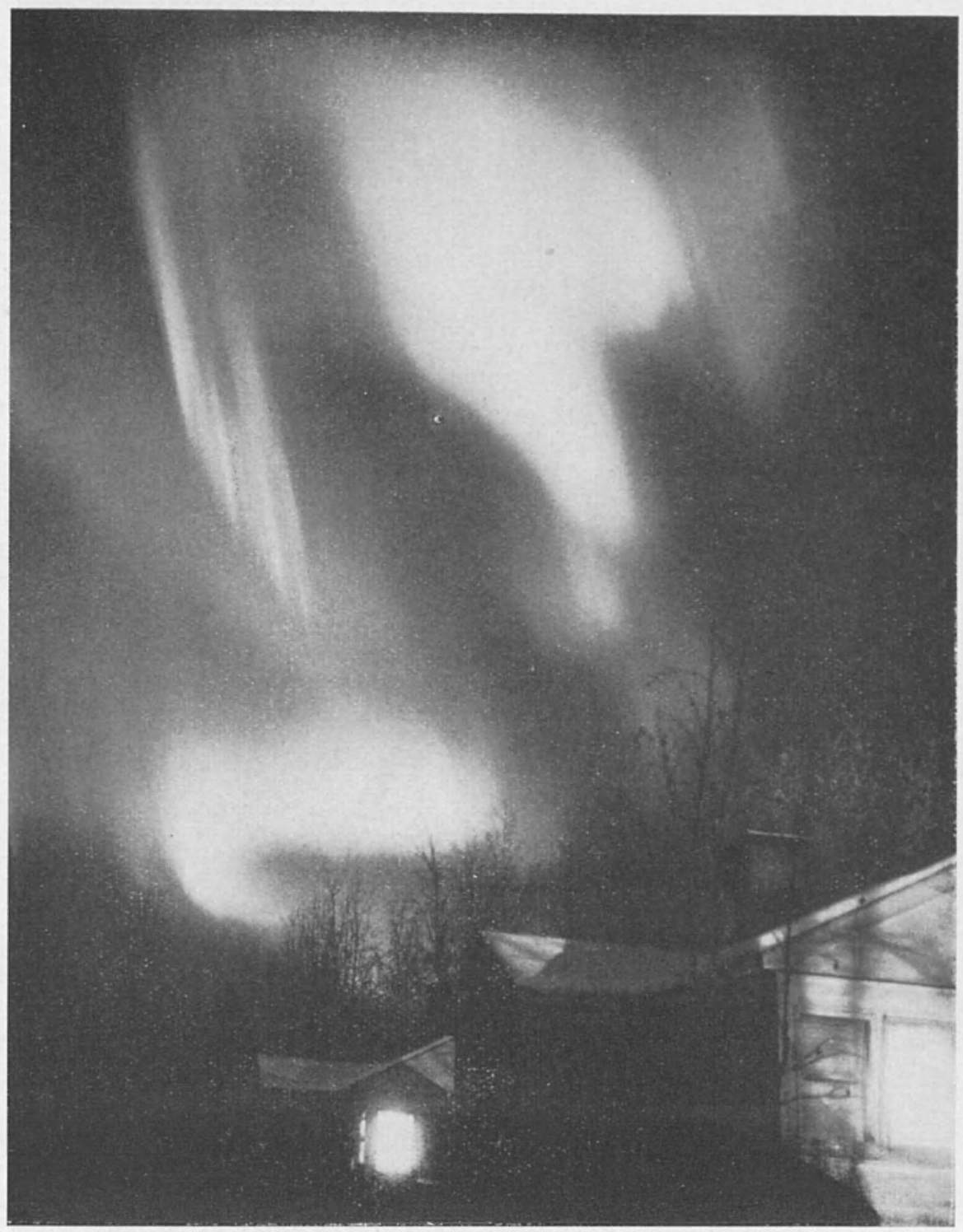

Drappeggio (per cortesia del dr. V. P. Hessler del Geophysical Institute University of Alaska, College). 


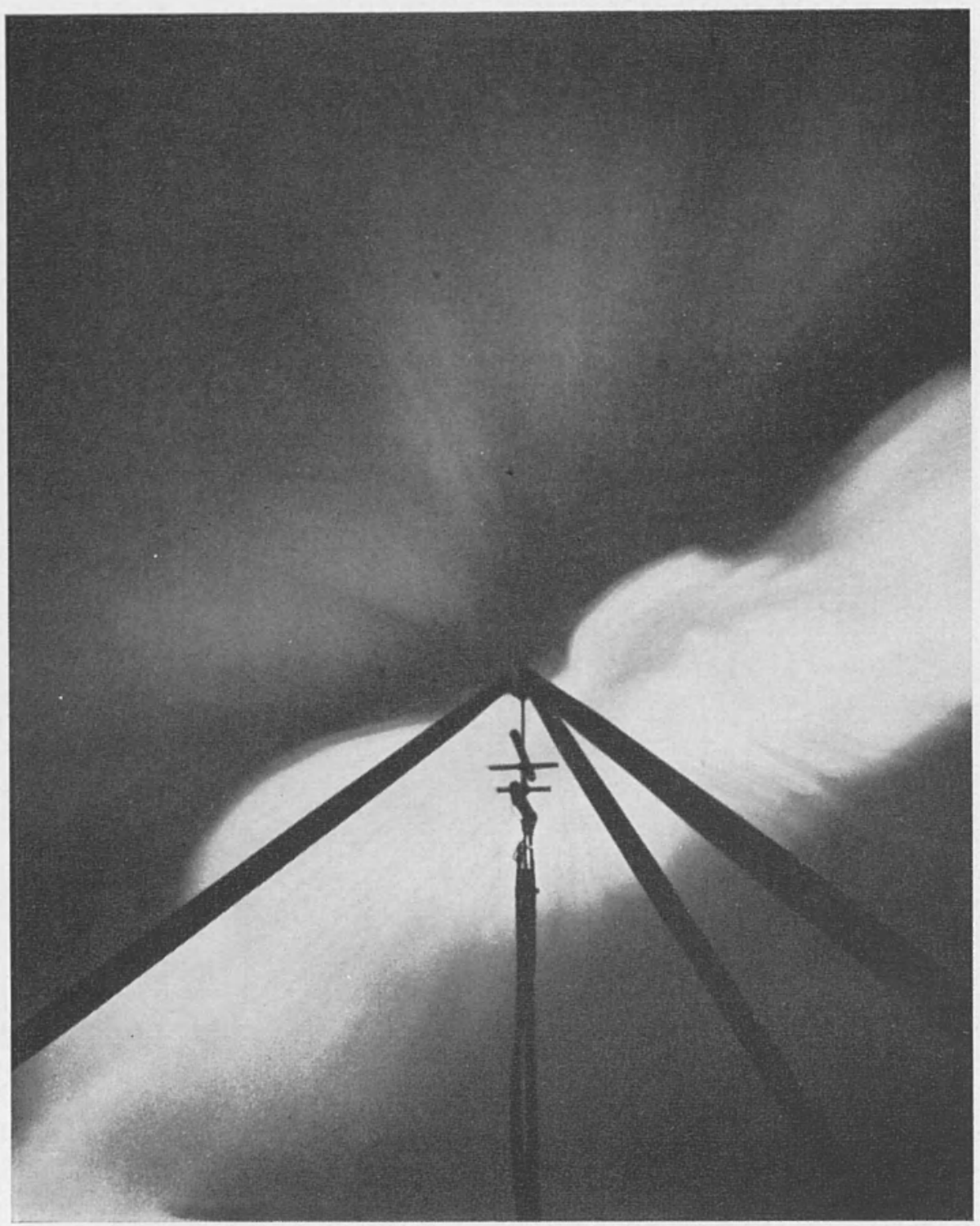

Corona (per contesia del dr. V. P. Ilessler del Cieophysical lustitite University of Alaska, College). 
riore di un arco situato al disotto dell'orizzonte. Nelle zone lontane dalle zone polari la colorazione in rosso è abbastanza frequente.

f) Superfici pulsanti (pulsating surfaces, PS). - Sono chiazze diffuse che ritmicamente, con periodo di qualche secondo, appaiono e scompaiono, conservando la loro forma irregolare. In prossimità dello zenith

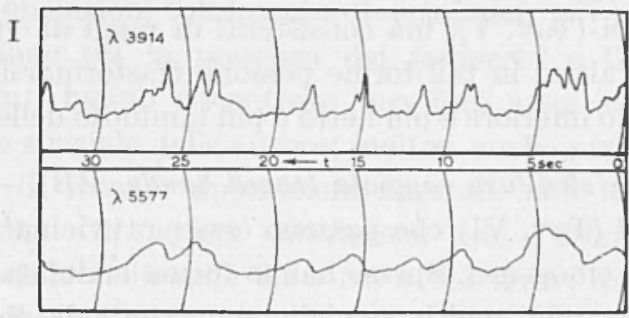

Fig. 2.1. - La intensità $I$ è in unità arbitrarie. Il ritardo d'emissione della riga $\lambda 5577$ rispetto alla banda $\lambda 3914$, è dovuto alla lunga vita media del livello ${ }^{1} S_{0}$ dell'atomo di ossigeno (per cortesia del dr. A. Omholt dell'Institutt for teoretisk astrofysikk, Oslo).

geomagnetico i contorni sono meglio delineati. Spesso le superfici pulsanti appaiono in concomitanza con le aurore fiammeggianti. La fig. 2.1 mostra la fluttuazione tipica di intensità della riga verde $\lambda 5577 \AA$ dell'ossigeno atomico e della banda $\lambda 3914$ del primo gruppo negativo del $\mathrm{N}_{2}^{+}$registrata all'Osservatorio di Yerkes.

2.1.2. Forme a struttura raggiata. - Sono forme costituite da raggi più o meno lunghi, più luminose delle forme a struttura non raggiata e spesso rapidamente mobili.

a) Raggi (rays, R). - Sono forme allungate (Tav. IV), isolate o anche assai prossime l'una dall'altra, di lunghezza e spessore assai vari. Possono restare stazionari per qualche minuto, muovendosi lentamente in senso est ovest o viceversa. Il colore può essere giallo, giallo-verde, grigio, e, nelle parti superiori, assai spesso rosso. La luminosità resta sensibilmente costante entro ampia parte della loro lunghezza.

I raggi possono vedersi dopo il tramonto o prima dell'alba anche nella parte di atmosfera illuminata dal Sole; tali forme (sunlit rays) si estendono fino a molte centinaia di $\mathrm{km}$ di altezza e più spesso hanno colore grigio-violetto, bleu, violetto e rosso. Altre strutture raggiate 
sono i raggi divisi (divided rays) che appaiono ai limiti del cono d'ombra della Terra: la loro parte inferiore si estende nella zona d'ombra e quella superiore, invece, nella zona illuminata dal Sole; le due parti sono separate da una zona oscura corrispondente al limite del cono d'ombra.

b) Archi raggiati (rayed arcs, RA). - Sono forme che richiamano gli archi omogenei (Tav. V), ma consistenti di raggi di varia lunghezza, l'uno accanto all'altro; in tali forme possono trasformarsi gli archi omogenei. Il loro bordo inferiore è più netto e più luminoso delle parti più alte.

c) Bande a struttura raggiata (rayed bands, $\mathrm{RB}$ ). - Consistono di una serie di raggi (Tav. VI) che possono essere ravvicinati l'uno all'altra lungo una banda omogenea. Spesso hanno forma ondulata o ad U; quando sono prossime allo zenith somigliano a ventagli. Spesso si hanno parecchie bande parallele l'una all'altra.

d) Drappeggi (draperies, D). - Sono forme rassomiglianti a un sipario (Tav. VII) con il bordo inferiore ondulato e più luminoso delle parti alte, che si hanno quando i raggi di una banda raggiata divengono molto lunghi. E frequente il caso di più drappeggi paralleli.

e) Corona (corona, C). - E la forma (Tav. VIII) che si osserva quando i raggi, comparendo in prossimità dello zenith, sembrano, per ragioni di prospettiva, tutti diretti verso un unico punto, detto punto di irradiazione. Tale punto in generale è prossimo allo zenith geomagnetico. I raggi possono circondare il punto di irradiazione in tutte le direzioni ovvero solo in alcune di esse. Analogo fenomeno prospettico può anche prodursi per altre forme aurorali che appaiono in prossimità dello zenith geomagnetico.

2.1.3. Aurore fiammeggianti (flaming aurorae, F). - Sono forme rapidamente variabili, costituite da intense onde di luminosità che si muovono verso l'alto una dopo l'altra, nella direzione dello zenith geomagnetico. Le aurore fiammeggianti sono frequenti dopo grosse aurore del tipo $\mathrm{R}$ o del tipo $\mathrm{D}$ e spesso sono seguite da formazione di corona.

2.1.4. La radioaurora. - Si indica con tale denominazione la intensa ionizzazione che si rileva mediante radiosondaggi con onde metriche alle quote e alle latitudini tipiche delle aurore visuali.

La prima notizia di echi radioelettrici osservati a Jodrell Bank risale al 1947: Lovell, Clegg e Ellyett $\left({ }^{3}\right)$ segnalarono di aver ricevuto 
echi discreti ed echi diffusi sulla frequenza di $46 \mathrm{MHz}$. Molti dei casi di anormali aumenti di ionizzazione nello strato $\mathrm{E}$, riscontrati da vari autori specialmente alle latitudini polari, sono stati attribuiti da Aspinall e Hawkins ( $\left.{ }^{4}\right)$ a riflessioni da radioaurore; gli autori hanno anzi riscontrato la presenza di due diversi tipi di echi, associati l'uno con gli archi e l'altro con i raggi. Occorre dire, tuttavia, che Harang e Landmark ( ${ }^{5}$ ) sono giunti a conclusioni totalmente diverse; essi, pur rilevando apprezzabile correlazione tra la presenza dei radioechi e l'attività aurorale (e geomagnetica), hanno riscontrato una loro assai scarsa correlazione con la posizione spaziale delle aurore; inoltre, anche puntando le antenne nella direzione di intense apparizioni aurorali, non hanno sempre osservato radioechi. Gli autori concludono con l'attribuire gli echi a retrodiffusione delle rudioonde da terra o dal mare, dopo riflessione su formazioni di strato $\mathrm{E}$ sporadico, le cui densità ioniche crescono sensibilmente durante le apparizioni aurorali e le tempeste magnetiche.

La radioaurora accompagna di norma l'aurora visuale; tuttavia, non sembra che ci sia sempre coincidenza nello spazio e nel tempo.

Dallo studio dei radioechi si riconosce che le densità elettroniche cui va attribuita la riflessione verso terra delle radioonde raggiungono $\left(^{3,8}\right)$ valori di $10^{7} \div 10^{8} \mathrm{~cm}^{-3}$, e finanche $10^{9} \div 10^{10} \mathrm{~cm}^{-3}$ nelle manifestazioni aurorali più intense. Una differenza tra $i$ radioechi provenienti dagli archi e dalle strutture raggiate è stata segnalata a College da Bowles ( $\left.{ }^{7}\right)$ : in effetti, l'autore, sperimentando con una frequenza di $25,4 \mathrm{MHz}$, ha rilevato la presenza di effetto Doppler nei radioechi, constatando che quelli corrispondenti a un moto verso il basso erano associati con le forme aurorali omogenee, mentre quelli corrispondenti a un moto verso l'alto erano associati con le forme aurorali raggiate.

Lo studio delle radioaurore costituisce un utile complemento a quello delle aurore visuali in quanto, mentre queste sono osservabili solo di notte e in condizioni di cielo favorevoli, le radioaurore lo sono per 24 ore su 24 .

\subsection{Distribuzione geografica e spaziale delle aurore. Movimenti.}

2.2.1. Distribuzione gcografica. - Di norma le aurore si osservano regolarmente nelle zone prossime ai circoli polari artico e antartico. Eccezionalmente si sono registrate aurore anche a latitudini molto basse, fin quasi all'equatore: così per es. il 4 Febbraio 1872 una aurora fu 
vista a Bombay (lat. geogr. $19^{\circ} \mathrm{N}$, lat. geomagn. $10^{\circ} \mathrm{N}$ ) e il 25 Settembre 1909 ne fu vista una a Singapore (lat. geogr. $1^{\circ} \mathrm{N}$, lat. geomagn. $10^{\circ} \mathrm{S}$ ).

Le zone di massima frequenza delle aurore sono due zone anulari, situate a una distanza angolare di circa $20 \div 25^{\circ}$ dai poli geomagnetici nord e sud, abbastanza simmetriche intorno ai poli stessi.

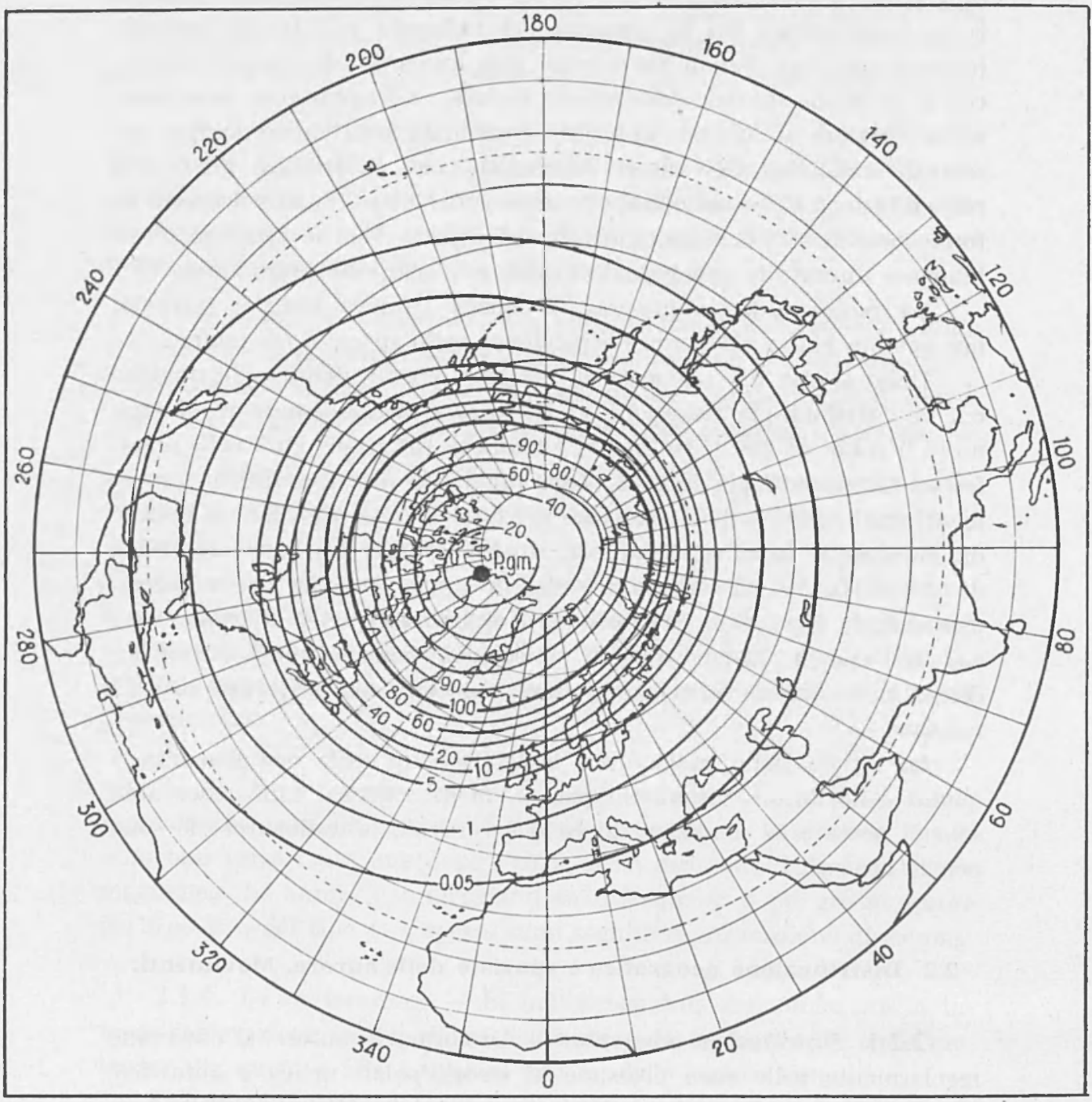

Fig. 2.2. - Isocasme per l'emisfero nord. P. gm. polo geomagnetico nord (secondo Vestine). 
Le fig. 2.2 e 2.3 mostrano le due più recenti carte delle isocasme (linee luogo dei punti ove uguale è la frequenza media di apparizione delle aurore), costruite da Vestine $\left(^{8}\right)$ e Vestine-Snyder $\left({ }^{9}\right)$ rispettivamente per gli emisferi nord e sud.

Ovviamente, nella costruzione delle carte intervengono numerose cause di imprecisione, tra cui, principali, la mancanza di risoluzione, specie in latitudine, nelle osservazioni aurorali e l'uso di risultati di osservazioni effettuate durante cicli solari differenti o in epoche diverse

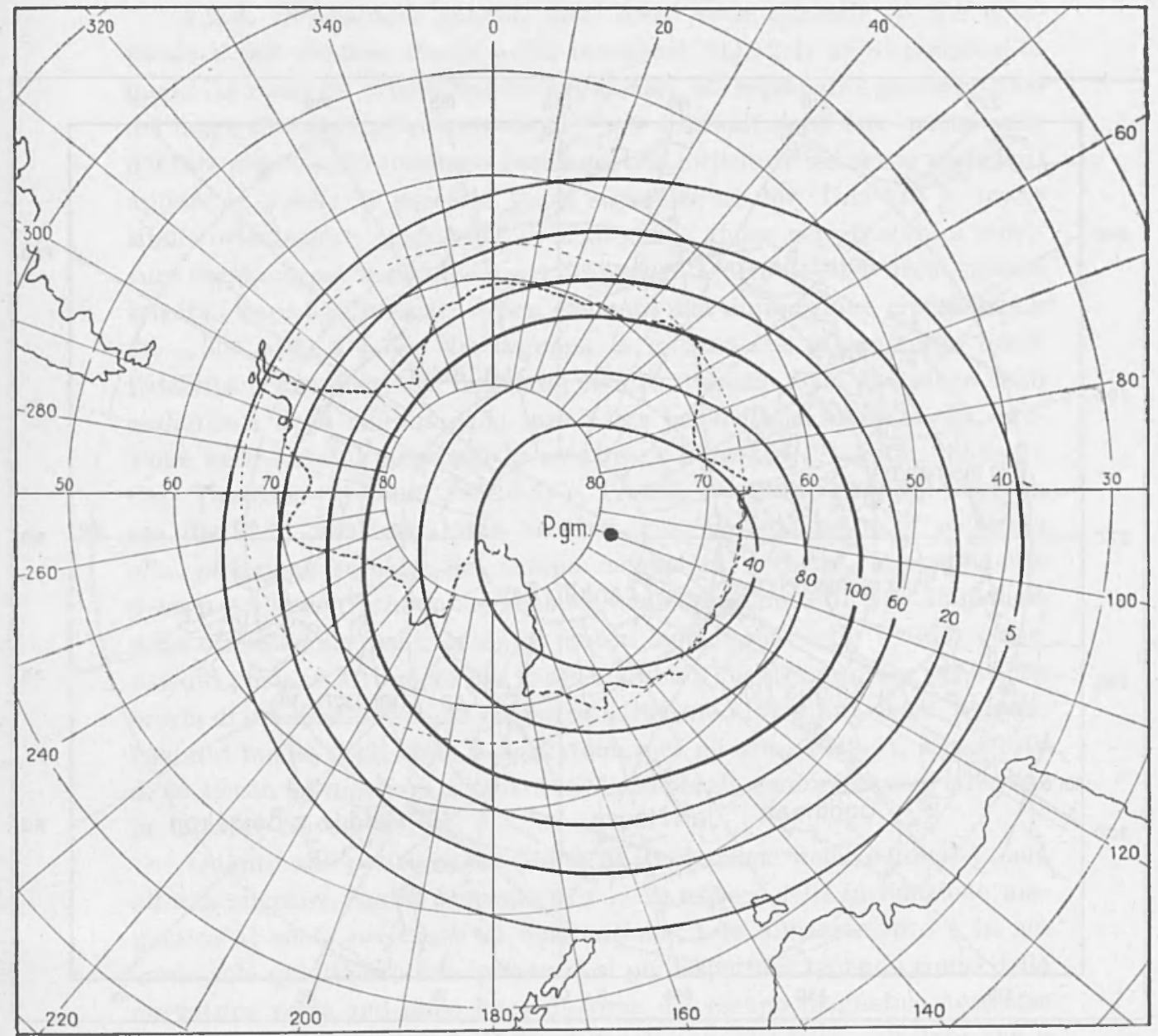

Fig. 2.3. - Isocasme per l'emisfero sud. P. gm. polo geomagnetico sud (secondo Vestine e Snyder). 
dello stesso ciclo; per l'emisfero sud si aggiunge inoltre la notevole scarsità delle osservazioni.

2.2.1.1. Associazioni tra aurore boreali e aurore australi. - Nel 1881, Fritz aveva ritenuto di poter stabilire che " grandi aurore nell'emisfero nord corrispondono sempre a grandi aurore nell'emisfero sud ». Solo recentemente la questione è stata ripresa da Little e Slirum ( ${ }^{10}$ ) che hanno confrontato le osservazioni aurorali effettuate dal 1938 al 1946 nella Nuova Zelanda e nella Regione dei Laghi di New York, a latitudini geomagnetiche corrispondenti: su 3285 giomi di osservazione in cui

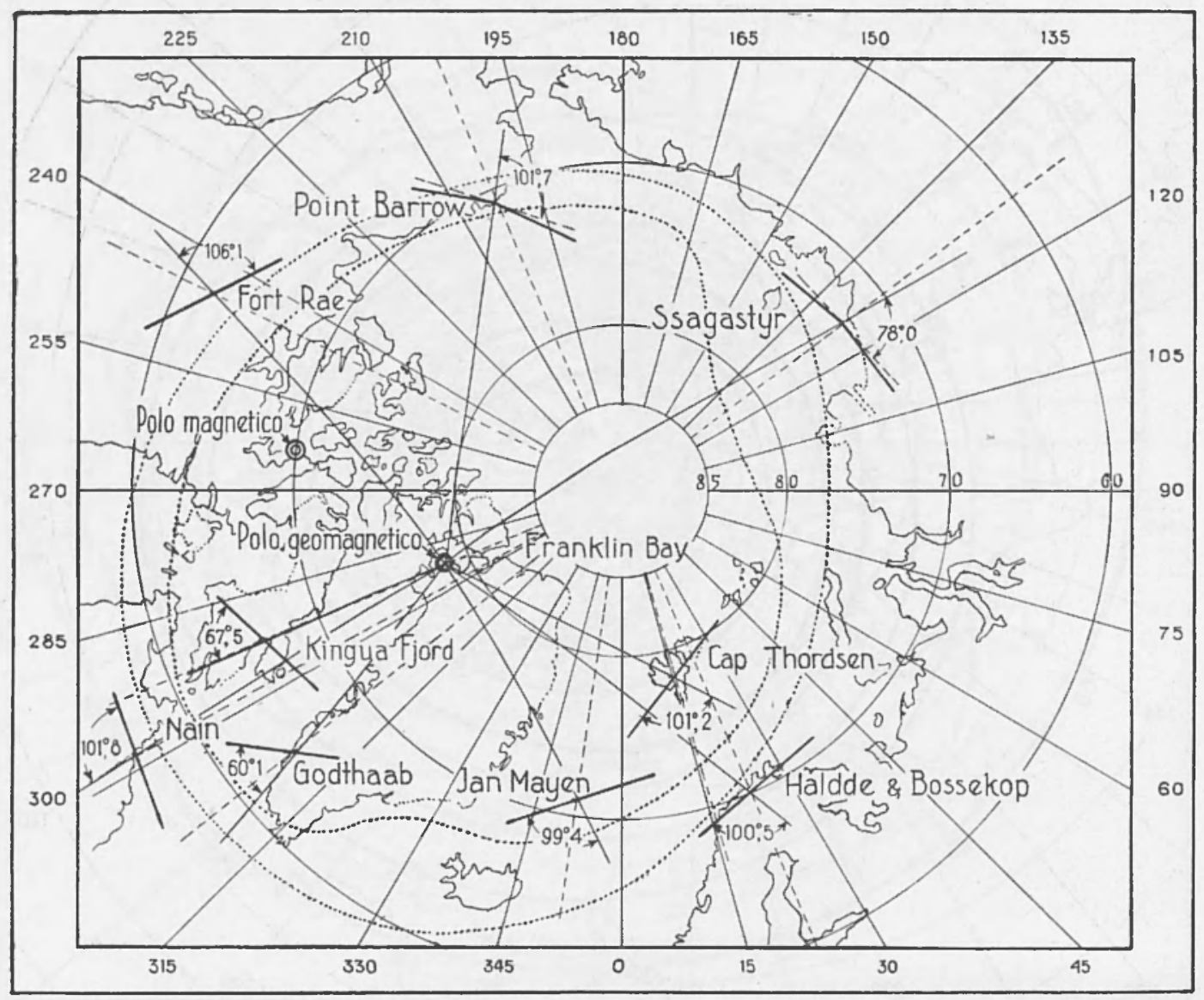

Fig. 2.4. - Orientazione degli archi (secondo Vegard e Krogness). 
vi furono 1316 giorni con aurora boreale e 863 con aurora australe, se ne ebbero 498 con aurore simultanee (entro l'intervallo tra le $0^{\text {h }}$ e le 12h di Greenwich).

La probabilità di apparizione di aurore simultanee risulta pertanto di circa $15 \%$; d'altra parte, la probabilità clie si trattasse di coincidenze casuali risulta del $10 \%$, cosicchè sembra di poter concludere clie una associazione tra aurore boreali e australi è assai dubbia; alla stessa conclusione giungono gli autori confrontando anche le intensità delle aurore.

2.2.2. Disposizione spaziale delle varie forme aurorali. $-\mathrm{Nel}$ complesso si può ritenere che gli archi omogenei (fig. 2.4) si dispongono in modo da risultare sensibilmente ortogonali al meridiano geomagnetico del luogo di osservazione: in effetti $\left({ }^{11},{ }^{12}\right)$ l'azimut della loro parte occidentale rispetto al meridiano geomagnetico (orientato nel senso nord-sud) appare in media di parecchi gradi superiore ai $90^{\circ}$. Una più o meno simile orientazione predominante si riscontra anche per gli arclii a struttura raggiata, per le bande e per i drappeggi. L'azimut degli archi subisce sensibili variazioni diurne: è ben evidente una diminuzione che giunge a $20 \div 30^{\circ}$ nelle $3-4$ ore che seguono la mezzanotte intorno alla quale l'attività geomagnetica è massima; inoltre, l'esame delle differenze negli andamenti degli azimut della loro parte occidentale rispetto alla direzione nord-sud del meridiano geografico a Bossekop (11) (anni 1838-40), Cap Thordsen $\left({ }^{13}\right)$ (anni 1882-83) e Tromsö ( ${ }^{14}$ ) (anni 1929-38) suggerisce (fig. 2.5) una variazione secolare che probabilmente è collegata alla variazione secolare del campo magnetico terrestre. Recentemente Jensen e Currie $\left({ }^{15}\right)$ hanno segnalato anche variazioni di tipo stagionale nella orientazione degli archi; gli autori aggiungono clie le loro osservazioni portano a ritenere per lo meno dubbia l'esistenza della variazione oraria di orientazione sopra riscontrata. Secondo i risultati di tali ricerche l'azimut medio degli arclii a Saskatoon, per gli anni 1949-51, è risultato di $96,4^{\circ}$ con un massimo di circa $115^{\circ}$ in Febbraio e un minimo di circa $86^{\circ}$ in Ottobre-Novembre.

Quanto alla posizione del punto di irradiazione della corona, la sua altezza angolare risulta di regola di $1 \div 2^{\circ}$ minore della inclinazione magnetica al suolo nel luogo di osservazione; tale abbassamento è in accordo solo qualitativo con quanto ci si può aspettare tenendo conto della curvaturn verso sud delle linee di forza del campo magnetico terrestre alle quote aurorali: in effetti Vegard e Krogness hanno calcolato che a $200 \mathrm{~km}$ da terra l'abbassamento delle linee di forza risulta di circa $0,1^{\circ}$. 
2.2.3. Distribuzione in altezza. - L'altezza minima a cui si manifestano i fenomeni aurorali è compresa di regola tra 80 e $100 \mathrm{~km}$. Quanto alla altezza massima, nella zona notturna della Terra essa raramente supera i $250 \div 300 \mathrm{~km}$; solo in casi eccezionali, in corrispondenza a grandi tempeste magnetiche, si sono osservate aurore fino a $700 \mathrm{~km}$. Ben

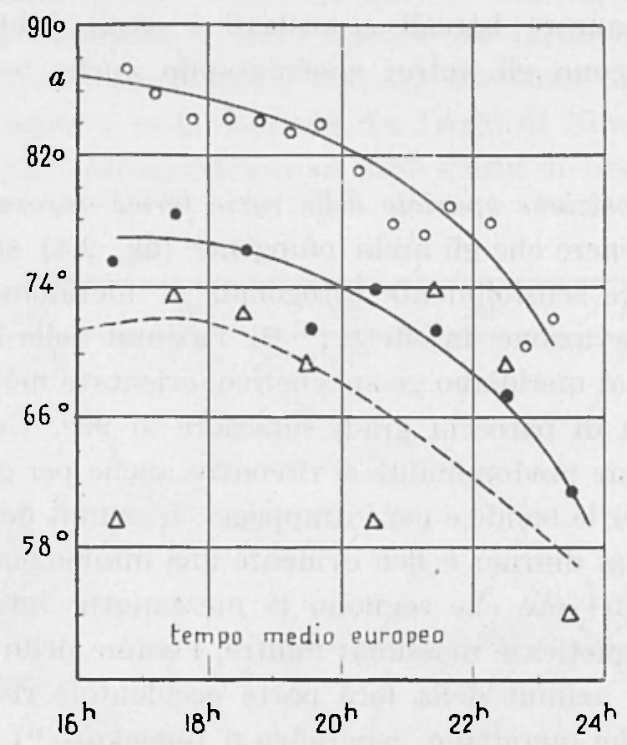

Fig. 2.5. - Andamenti temporali degli azimut $a$, rispettivamente a Bossekop (ooo), Cap Thordsen $(\Delta \Delta \Delta)$ e Tromsö $(\cdots)$. (secondo Harang).

più frequenti sono le osservazioni di forme aurorali a grandi altezze nella atmosfera illuminata dai raggi solari al crepuscolo (sunlit auroras).

Le osservazioni più numerose si riferiscono ovviamente alle forme aurorali notturne.

Le figg. $2.6 a$ e $2.6 b$ riportano la frequenza di occorrenza dei limiti inferiori dei differenti tipi di aurore, osservate all'Osservatorio di Haldde, nella Norvegia settentrionale, nel 1913-14 secondo una statistica dovuta a Vegard e Krogness (11); la tabella 2.1 riporta le medie pesate $H$ delle altezze minime per i vari tipi, il numero $N$ di osservazioni e la media pesata complessiva.

Le determinazioni di altezza delle aurore osservate in Norvegia sono generalmente in accordo con quelle fatte da altri autori in altri luoghi: così Currie (16) a Chesterfield Inlet, in Canada, ha ottenuto negli 

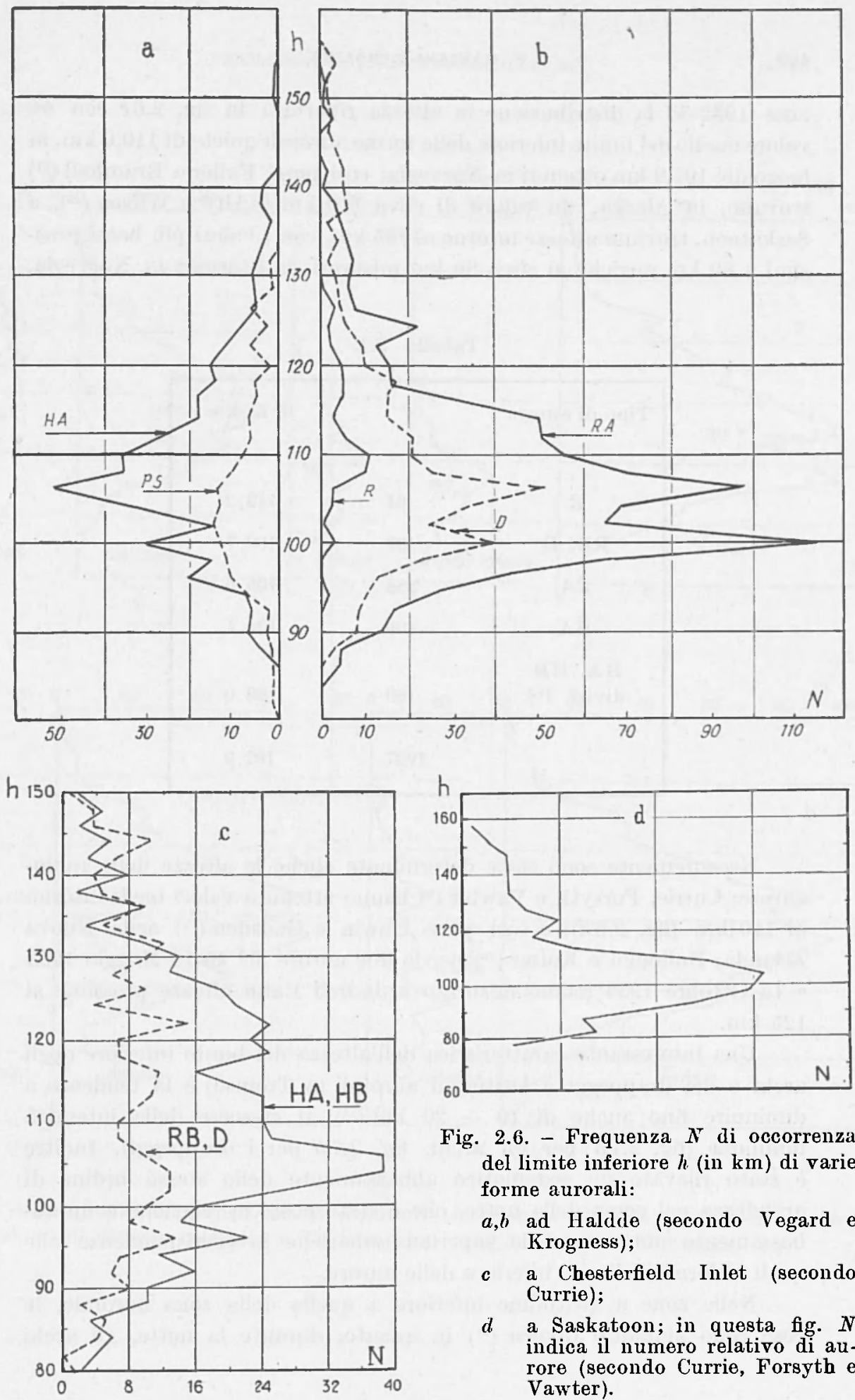

Fig. 2.6. - Frequenza $N$ di occorrenza del limite inferiore $h$ (in $\mathrm{km}$ ) di varie forme aurorali:

$a, b$ ad Haldde (secondo Vegard e Krogness);

c a Chesterfield Inlet (secondo Currie);

$d$ a Saskatoon; in questa fig. $N$ indica il numero relativo di aurore (secondo Currie, Forsyth e Vawter). 
anni 1932-33 la distribuzione in altezza riportata in fig. $2.6 c$ con un valore medio del limite inferiore delle forme aurorali quiete di $110,0 \mathrm{~km}$, in luogo dei 107,9 km ottenuti in Norvegia; cosi pure, Fuller e Bramhall ( ${ }^{17}$ ) trovano, in Alaska, un valore di circa $110 \mathrm{~km}$ e Alty e Wilson ( ${ }^{18}$ ), a Saskatoon, trovano altezze intorno ai $105 \mathrm{~km}$, con i valori più bassi prossimi a $60 \mathrm{~km}$ anzichè ai circa $80 \mathrm{~km}$ misurati da Stormer in Norvegia.

Tabella 2.1.

\begin{tabular}{|c|c|c|}
\hline Tipo di aurora & $N$ & I in km \\
\hline R & 61 & 113,2 \\
RB, D & 409 & 109,7 \\
RA & 888 & 106,6 \\
HA & 409 & 109,1 \\
HA, HB & & 106,0 \\
divisi, PS & 160 & 107,9 \\
\hline
\end{tabular}

Recentemente sono state determinate anche le altezze delle radioaurore: Currie, Forsyth e Vawter $\left(^{6}\right)$ hanno ottenuto valori medi intorno ai $110 \mathrm{~km}$ (fig. $2.6 d$ ); e cosi pure Unwin e Gadsden (19) nella Nuova Zelanda; Bullough e Kaiser ${ }^{(20}$ ) per le due aurore del 15-16 Maggio 1953 e 15 Ottobre 1953 hanno misurato a Jodrell Bank altezze prossime ai $125 \mathrm{~km}$.

Una interessante caratteristica dell'altezza del bordo inferiore degli archi e dei drappeggi a latitudini aurorali (a Tromsö) è la tendenza a diminuire fino anche di $10 \div 20 \mathrm{~km}\left({ }^{14}\right)$ al crescere della intensità luminosa (fig. $2.7 a$ per gli archi, fig. $2.7 b$ per i drappeggi). Inoltre è stato rilevato un sistematico abbassamento dello stesso ordine di grandezza nel corso della notte, che è stato posto in relazione a un abbassamento notturno delle superfici isobariche in corrispondenza alle quali si forma il limite inferiore delle aurore.

Nelle zone a latitudine inferiore a quella della zona aurorale, le cose sono alquanto diverse ${ }^{21}$ ) in quanto, durante la notte, gli archi 

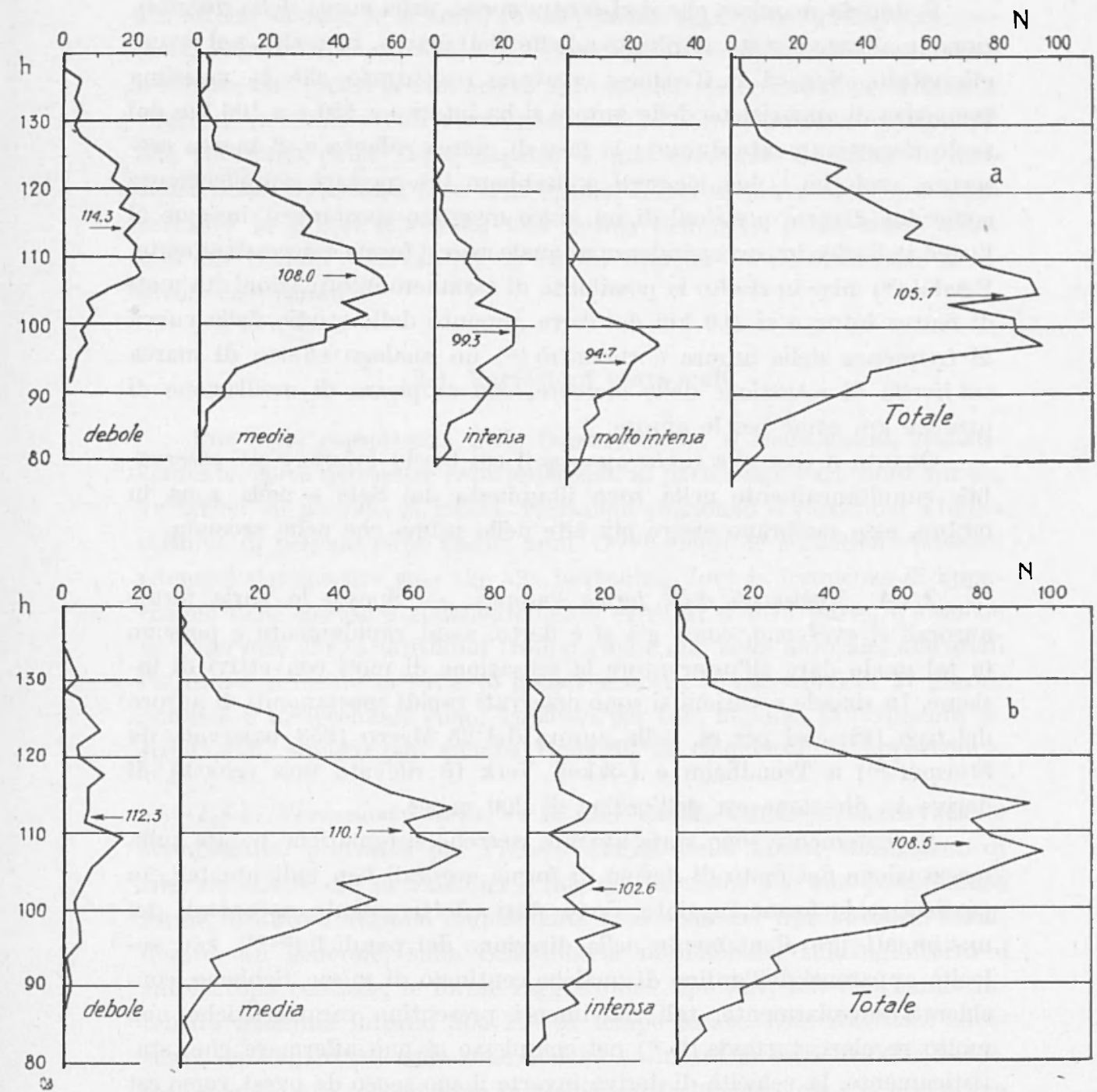

Fig. 2.7. - Frequenza $N$ di occorrenza del limite inferiore $h$ (in $\mathrm{km}$ ) per archi $(a)$ e drappeggi $(b)$ di diversa intensità a Tromsö, anni 1929-38 (secondo Harang). 
omogenei restano praticamente sempre alla stessa quota, mentre le forme raggiate tendono ad abbassarsi notevolmente, finanche di $30 \mathrm{~km}$.

Il doppio massimo che si riscontra spesso nella curra della distribuzione in altezza è stato attribuito ad effetti di marea. In realtà, nel lavoro già citato, Vegard e Krogness avevano riscontrato che la massima frequenza di apparizione delle aurore si ha intorno a 100 e a $106 \mathrm{~km}$ dal suolo rispettivamente durante la fase di marea calante e di marea crescente, cosicchè $\mathrm{i}$ due massimi andrebbero interpretati semplicemente come due diverse posizioni di un unico massimo spostantesi insieme al livello isobarico in corrispondenza al quale esso si forma. Successivamente, Egedal $\left({ }^{22}\right)$ mise in risalto la possibilità di assumere informazioni sui moti di marea intorno ai $100 \mathrm{~km}$ da Terra appunto dallo studio della curva di frequenza delle aurore e riscontrò $\left({ }^{23}\right)$ un analogo effetto di marea sul livello di estinzione delle meteore, con ampiezza di oscillazione di qualche $\mathrm{km}$ come per le aurore.

Quanto invece alle sunlit aurorae il cui bordo inferiore sia osservabile simultaneamente nella zona illuminata dal Sole e nella zona in ombra, esse sembrano essere più alte nella prima che nella seconda.

2.2.4. Movimenti delle forme aurorali. - Spesso le varie forme aurorali si evolvono, come già si è detto, assai rapidamente e possono in tal modo dare all'osservatore la sensazione di moti convettivi di insieme. In singole occasioni si sono osservati rapidi spostamenti di aurore del tipo DS: così per es. nella aurora del 25 Marzo 1953 osservata da Stormer (21) a Trondheim e Lökken Verk fu rilevata una velocità di deriva in direzione est dell'ordine di $200 \mathrm{~m} / \mathrm{sec}$.

Recentemente sono state avviate ricerche sistematiche basate sulla osservazione del moto di deriva di forme aurorali ben individuabili, in particolare le forme raggiate. Sono stati effettivamente riscontrati dei movimenti prevalentemente nella direzione dei paralleli $\left({ }^{25},{ }^{26}\right)$, con velocità apparenti dell'ordine di qualche centinaio di $\mathrm{m} / \mathrm{sec}$. Sebbene, considerati singolarmente, tali movimenti presentino caratteristiche non molto regolari, tuttavia $\left(^{25,}{ }^{27}\right)$ nel complesso si può affermare che, statisticamente, la velocità di deriva inverte il suo senso da ovest verso est a est verso ovest intorno alla mezzanotte locale, ciò che potrebbe essere in connessione con l'analogo fenomeno di inversione che pure si riscontra intorno alla mezzanotte nelle correnti ionosferische che determinano la variazione $S_{D}$ del campo magnetico terrestre (v. 1.3.3). Tuttavia recenti misure di Kim e Currie ${ }^{(28)}$ non sembrano confermare tale inversione di velocità. 
Si discute tuttora sulla interpretazione fisica delle osservate velocità di deriva: se cioè si tratti di effettivi movimenti convettivi della atmosfera, oppure se si tratti di movimenti apparenti dovuti a un graduale spostamento rispetto alla Terra delle aree colpite dalla radiazione incidente che eccita la luminosità aurorale. Le determinazioni di Kim e Currie non hanno rivelato movimenti delle forme aurorali attribuibili alla rotazione della Terra rispetto a una eventuale direzione di incidenza della radiazione, fissa nello spazio. L'ipotesi più probabile sembra pertanto la prima; tuttaria, una parola definitiva potrà essere detta solo effettuando osservazioni di effetti Doppler in determinate. favorevoli condizioni.

\subsection{Variazioni temporali}

Pur nellia complessità della fenomenologia si riconoscono, statisticamente, certe ricorrenze caratteristiche: in particolare variazioni diurne, variazioni di periodo 27 giorni, variazioni stagionali e variazioni a lungo termine di periodo circa undici anni. Ovviamente le statistiche possono ritenersi significative solo alle alte latitudini, dove la frequenza di apparizione delle aurore è sufficientemente elevata; d'altrác parte, c'è anche da osservare che, a latitudini troppo vicine alla zona aurorale, alle quali l'aurora è presente in tutte (o quasi) le notti, le variazioni di 27 giorni, annuale e undecennale sono, appunto per tale ragione, difficilmente riscontrabili, almeno per quanto riguarda la frequenza di apparizione.

2.3.1. Variazioni diurne. - L'osservazione visuale e l'osservazione strumentale, protratte per l'intera durata della notte, consentono di rilevare che le ore di massima e minima intensità per una determinata forma di aurora variano ampiamente a seconda del tipo aurorale considerato. In generale, sulla Scandinavia meridionale, sull'Inghilterra e sull'Europa centrale, le forme raggiate del tipo $\mathrm{RA}, \mathrm{RB}$ e $\mathrm{C}$ hanno intensità massima intorno alle $22^{\mathrm{h}}$ di tempo locale. Una statistica effettuata da Vegard $\left({ }^{20}\right)$ nelle zone artiche ha messo in evidenza che le forme raggiate e le corone sono in media più frequenti circa 1,3 ore prima della mezzanotte geomagnetica locale (v. 1.3.2). Per le forme intense e brillanti o, rispettivamente, deboli e quiete, Fuller $\left({ }^{30}\right)$ ha trovato, a College, che le prime sono più frequenti nelle ore serali e le altre nelle ore mattutine.

Quanto alle radioaurore, l'andamento diurno delle frequenze di apparizione (fig. 2.8) osservate negli Stati Uniti e in Canada mostra 
caratteristiche notevolmente interessanti: pur essendo possibile, come grià si è detto, tenere in esame la radioaurora per 24 ore su 2:1, risulta tuttavia che essa è presente di regola solo dopo le $14^{\text {h }}$ di tempo locale, con un massimo intorno alle $20^{\mathrm{h}}$, e fino alle $4^{\text {h }}$ del mattino. Questa caratteristica della radioaurora di essere praticamente assente nelle ore antimeridiane richiama l'analoga caratteristica delle perturbazioni magnetiche polari (v. 1.3.5).

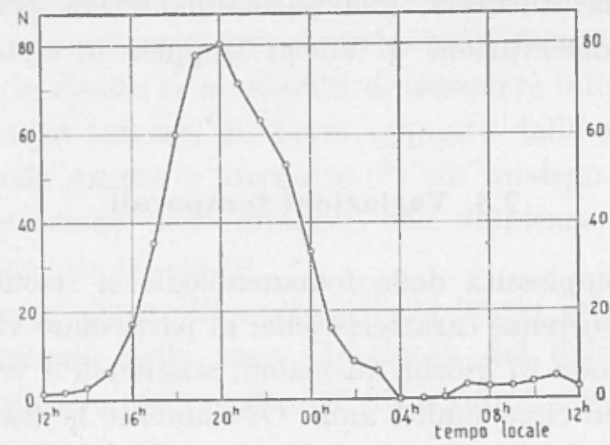

Fig. 2.8. - Variazione diurna della frequenza $N$ di osservazione della radioaurora negli anni 1949.5 I (secondo Gerson).

Tutto ciò suggerisce che nelle prime ore del mattino agisca qualche mecanismo, nell'atmosfera o fuori di essa, che tende a impedire forti aumenti di ionizzazione: potrebbe trattarsi di un effetto connesso con l'azione della radiazione ultravioletta solare (peraltro non chiaramente immaginabile) oppure di un effetto dovuto alla precessione del polo geomagnetico intorno al polo geografico che, nella plausibile ipotesi che l'aurora sia prodotta da un fascio di particelle cariche, influirebbe sulle orbite di queste.

2.3.2. Variazione di periodo 27 giorni. - Le aurore di moderata entità mostrano, secondo Fritz $\left({ }^{\mathrm{C}}\right)$ e Sverdrup $\left({ }^{31}\right)$, tendenza a ricorrere dopo circa 27 giorni, in presumibile relazione con il riapparire sul Sole di un centro perturbato $\mathrm{o}$, più probabilmente, di una regione $\mathbf{M}$ dopo una sua intera rotazione.

In una recente analisi statistica di 1267 aurore osservate negli anni 1897-1951 all'Osservatorio di Yerkes (lat. geomagn. $53^{\circ} \mathrm{N}$ ), Meinel, Negaard e Chamberlain $\left({ }^{32}\right)$, studiando le ricorrenze di periodo da 1 a 

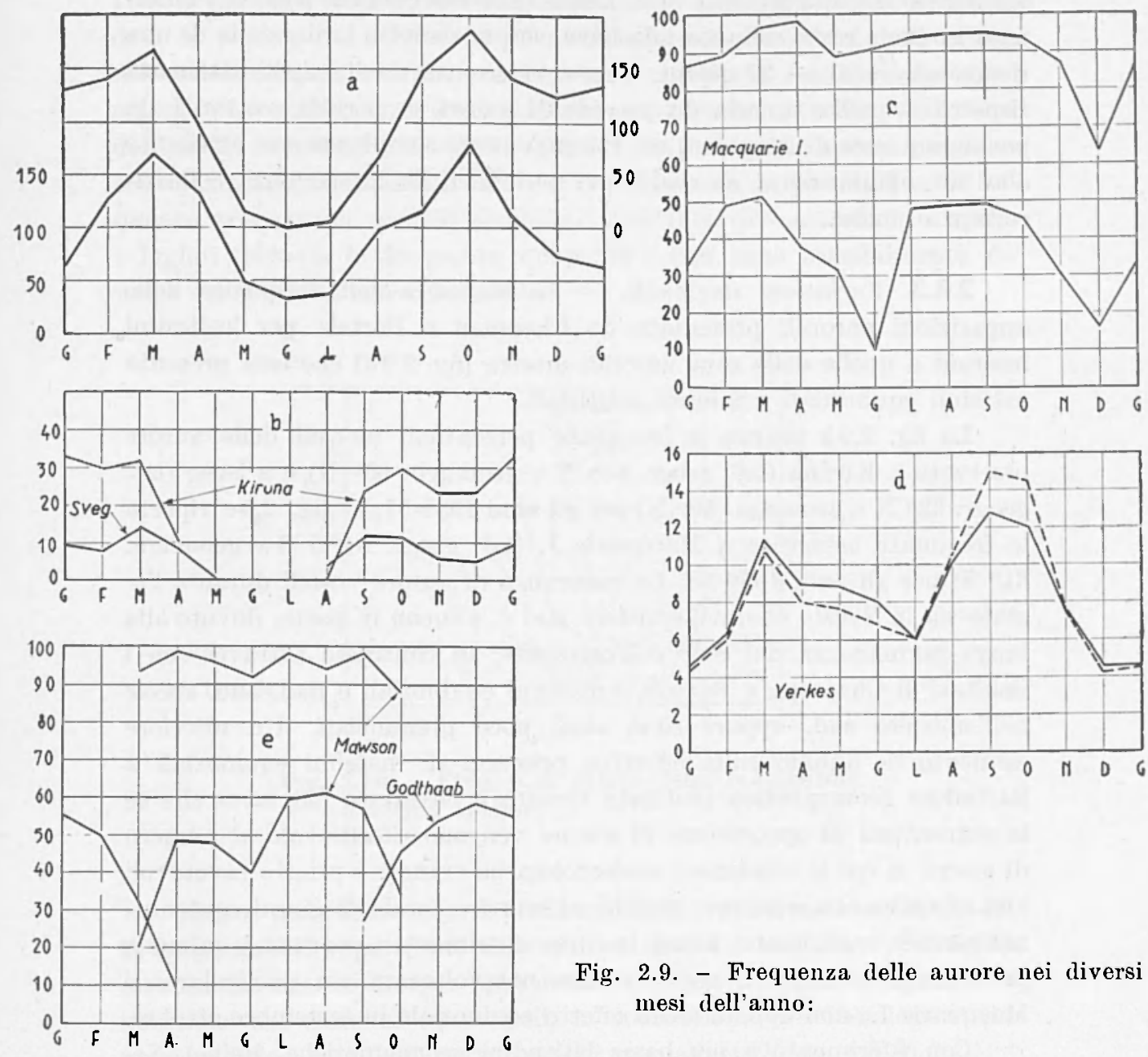

Fig. 2.9. - Frequenza delle aurore nei diversi mesi dell'anno:

$a$ in Svezia (curva superiore) e nell'emisfero sud (curva inferiore); le curve sono normalizzate in modo che la media annuale sia uguale a 100 (secondo Chapman e Bartels);

$b$ a Kiruna e Sveg;

c,e a Macquarie I. e a Mawson; curva inferiore, frequenza osservata; curva superiore, frequenza corretta;

$d$ a Yerkes; curva tratteggiata, percentuale del numero annuo totale; curva continua, percentuale corretta (secondo Meinel, Negaard e Chamberlain). 
400 giorni, hanno rilevato una ricorrenza principale di circa 29,5 giorni, attribuita al disturbo della luce lunare sulle osservazioni aurorali, eliminata la quale resta soltanto una lieve, se pur definita indicazione di una ricorrenza di $26 \div 27$ giorni. Questa ricorrenza risulta assai attenuata rispetto a quella trovata da precedenti autori su periodi costituiti da poche sequenze di 27 giorni, ciò che può essere semplicemente attribuito alla non eliminazione, su così brevi periodi, della interferenza colla ricorrenza lunare.

2.3.3. Variazioni stagionali. - La statistica della frequenza delle apparizioni aurorali presentata da Chapman e Bartels per latitudini inferiori a quelle delle zone aurorali mostra (fig. $2.9 a$ ) che essa presenta massimi equinoziali e minimi solstiziali.

La fig. $2.9 b$ mostra le frequenze percentuali mensili delle aurore osservate a Kiruna (lat. geogr. 68 $\mathrm{N}$ e geomagn. $65^{\circ} \mathrm{N}$ ) e a Sveg (lat. geogr. $62^{\circ} \mathrm{N}$ e geomagn. $59^{\circ} \mathrm{N}$ ) per gli anni 1939-57; la fig. $2.9 c$ riporta le frequenze osservate a Macquarie I. (lat. geogr. $54^{\circ}, 5 \mathrm{~S}$ e geomagn. $\left.61^{\circ} \mathrm{S}\right)$ per gli anni 1950-58. La mancanza di aurore visuali durante l'estate sia in Svezia che nell'emisfero sud è, almeno in parte, dovuto alla lunga permanenza del Sole sull'orizzonte; in contrasto tuttavia con i risultati di Chapman e Bartels, i massimi equinoziali o mancano, specie nell'emisfero sud, oppure sono assai poco pronunciati. Un ulteriore elemento di dubbio sulla effettiva presenza di massimi equinoziali a latitudine geomagnetica piuttosto elevata è costituito dal fatto che se le percentuali di apparizione di aurore vengono riferite solo al numero di giorni in cui le condizioni meteorologiche erano a "priori " favorevoli alla effettiva osservazione, anzichè al numero totale di giorni contenuti nel periodo considerato, allora le curve sperimentali " corrette " (almeno per l'emisfero sud) si appiattiscono notevolmente: in particolare, a Macquarie I., non appare alcun effetto equinoziale in settembre-ottobre.

Con riferimento a più basse latitudini geomagnetiche, Meinel, $\mathrm{Ne}$ gaard e Chamberlain, nel lavoro già citato $\left({ }^{32}\right)$, hanno riscontrato (figura $2.9 d$ ) la chiara presenza di due massimi equinoziali della frequenza percentuale di occorrenza delle aurore all'Osservatorio di Yerkes (anni 1897-1951), di cui quello autunnale prevalente su quello primaverile, anche tenendo opportunamente conto degli effetti meteorologici e della diversa durata della notte nei diversi mesi dell'anno.

All'interno delle zone aurorali (a latitudini superiori ai $70^{\circ}$ circa) la situazione sembra alquanto diversa: la fig. $2.9 d$ riporta le frequenze percentuali mensili delle aurore osservate ${ }^{33}$ ) a Godthaab (lat. geomagn. 
$76^{\circ} \mathrm{N}$ ) negli anni 1865-82 e a Mawson (lat. geomagn. $73^{\circ} \mathrm{S}$ ) negli anni 1954-58: le osservazioni, oltrechè scarse, sono limitate ai mesi invernali, data la presenza continua del Sole sull'orizzonte durante i mesi estivi; nell'emisfero nord sembra presente un andamento con un solo netto massimo invernale, anzichè un minimo come sembra avvenire al di fuori dell'anello aurorale; nell'emisfero sud i dati di osservazione sono ancora più scarsi che nell'emisfero nord; a Mawson, in particolare, le frequenze percentuali mensili sembrano mostrare due massimi in aprile e luglio; tuttavia le frequenze "corrette" non sono sensibilmente diverse nei mesi da marzo a ottobre.

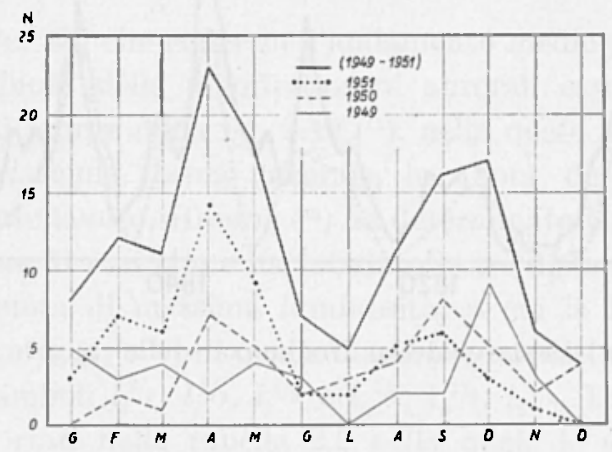

Fig. 2.10. - Frequenza oraria delle radioaurore (secondo Gerson).

Si può concludere che il problema della variazione stagionale della frequenza di apparizione delle aurore visuali è tutt'altro che risolto; esso merita uno studio più approfondito, specie per quanto riguarda la questione dei massimi equinoziali, in connessione con l'analoga caratteristica presentata dalle perturbazioni geomagnetiche (v. 1.5.3).

Per quanto concerne invece la radioaurora, le indicazioni speririmentali sono notevolmente più significative, sopratutto per il fatto di non essere influenzate da fattori meteorologici; la fig. 2.10 mostra il numero $N$ di giorni in cui essa fu osservata da Gerson $\left({ }^{34}\right)$, separatamente per gli anni 1949, 50 e 51 e, complessivamente negli anni 1949-51. $\Delta$ differenza delle aurore visuali, sono ben evidenti due massimi in aprile e in ottobre: occorre tuttavia tenere presente che le osservazioni di Gerson si riferiscono a latitudini geomagnetiche piuttosto basse (tra 50 e $60^{\circ} \mathrm{N}$ ). 
2.3.4. Variazioni di periodo undici anni. - In linea generale si riscontra un certo parallelismo di comportamento tra numero di Wolf $R$ e numero di giorni $N$ in cui per ogni anno si sono osservati fenomeni aurorali. La fig. 2.11 per es. mostra l'andamento di $N$ e $R$ per gli anni 1780-1877 ottenuto da Harang su dati raccolti da Tromholt (35) per la Norvegia; risultati sostanzialmente analoghi sono stati ottenuti, per gli anni 1840-96 da Boller ${ }^{\left({ }^{38}\right)}$ per l'emisfero Sud.

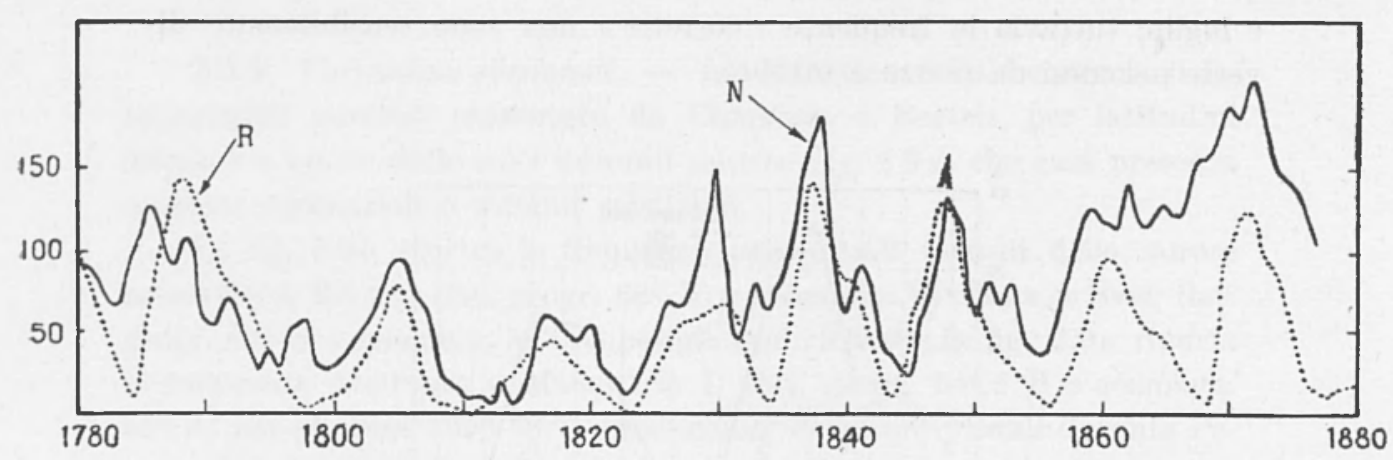

Fig. 2.11. - Variazione secolare della frequenza delle aurore (secondo Tromholt).

Questi e altri analoghi risultati suggeriscono che, a latitudini inferiori a quelle delle zone aurorali, la massima frequenza di apparizione si ha all'incirca un anno o due dopo il massimo dell'attività solare, sia nell'emisfero nord $\left({ }^{37}\right)$ che nell'emisfero sud $\left({ }^{38}\right)$.

A latitudini superiori a quelle delle zone aurorali, di quella artica almeno, si ha invece un comportamento inverso, con massimi di frequenza all'incirca corrispondenti a minimi di attività solare e viceversa $\left({ }^{39}\right)$.

C'è tuttavia da rilevare che gli andamenti di $N$ per gli Osservatori di Kiruna, Sveg, Macquarie I. e Mawson, negli stessi periodi sopra considerati, presentano una correlazione piuttosto scarsa con l'andamento di $R$, la qual cosa fa nascere dei dubbi sul reale significato fisico delle statistiche di Harang e Boller.

L'opposto comportamento entro e fuori le zone aurorali sarebbe probabilmente dovuto al fatto che, man mano che cresce l'attività solare, le zone stesse tendono a spostarsi verso latitudini inferiori, mentre il contrario avviene al diminuire dell'attività. Così pure ad un analogo spostamento va attribuito il massimo della frequenza aurorale che si ha d'inverno all'interno della zona aurorale, di cui si è parlato in 2.3.3. 
2.3.5. Altre variazioni periodiche. - Aleuni autori hanno ritenuto di poter individuare altre periodicità nelle manifestazioni aurorali: cosi Schmidt ${ }^{\left({ }^{40}\right)}$ e Angenheister ${ }^{(11}$ ) segnalarono, in relazione ad una presunta analoga periodicità delle perturbazioni geomagnetiche, una periodicità aurorale di 30 giorni, che peraltro un'approfondita analisi critica non consente di ritenere sufficientemente provata. Fritz, inoltre, dall'esame di una lunga serie di osservazioni, ha riscontrato una periodicità di circa 55 anni, comprendente cinque periodi di 11 anni.

\subsection{Luminosità e spettro delle aurore}

2.4.1. - Per ciò che concerne l'andamento medio con la quota dell'intensità luminosa delle manifestazioni aurorali esso è rappresentato per vari tipi di aurore dalla fig. $2.12\left({ }^{11}\right)$, nella quale la quota zero rappresenta, per ciascuna forma aurorale, la quota del bordo inferiore.

In un recente lavoro, Harang ${ }^{(2)}$ ) ha determinato la curva di luminosità per 54 aurore di vari tipi e ha tabulato le tre distanze al disotto e al disopra della quota di massima luminosità, a cui la luminosità stessa appare ridotta a $2 / 3,1 / 2$ e $1 / 3$ di quella massima; tali distanze (in $\mathrm{km}$ ) sono indicate con i simboli $l_{l}^{2 / 3}, l_{l}^{1 / 2}, l_{l}^{1 / 3}$ e $l_{u}^{1 / 3}, l_{u}^{1 / 2}, l_{u}{ }^{2 / 3}$. I risultati della statistica sono riportati nella tabella 2.2 nella quale le aurore sono state ripartite in gruppi a seconda della quota $h_{\max }$ di massima luminosità.

2.4.2. Interpretazione fisica delle curve di luminosità. - La luminosità aurorale è determinata dalla eccitazione degli atomi e delle molecole costituenti l'alta atmosfera da parte di una radiazione incidente. Questa potrebbe essere a priori di natura sia elettromagnetica che corpuscolare. Tuttavia, la presenza notturna delle aurore e il netto effetto di latitudine che esse presentano permettono senz'altro di escludere che le aurore stesse siano provocate da radiazione elettromagnetica primaria di origine solare; inoltre, lo stesso effetto di latitudine e il chiaro collegamento delle principali forme aurorali con il campo geomagnetico (v. 2.2.2) portano a concludere in favore della ipotesi di una radiazione primaria corpuscolare costituita da particelle dotate di carica elettrica.

Già un semplice studio dell'andamento della luminosità aurorale in funzione della quota fornisce importanti notizie sulla natura fisica e, una volta nota la composizione dell'atmosfera al variare della quota, sulla energia delle particelle incidenti. Le prime ricerche in tal senso si debbono a Lenard $\left({ }^{43}\right)$ il quale, nella semplice quanto ragionevole 
ipotesi che la luminosità prodotta da un fascio rettilineo di elettroni sia ad ogni quota proporzionale alla perdita di energia per unità di lunghezza, trovò $\left({ }^{*}\right)$, per atmosfera isoterma, che il numero $L$ di fotoni emessi

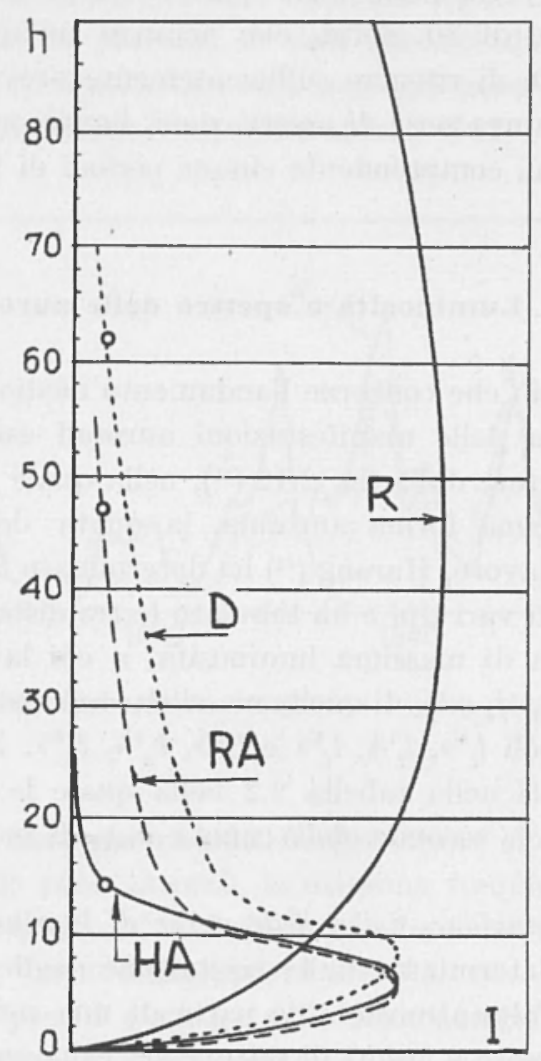

Fig. 2.12. - L'altezza $h$ è in $\mathrm{km}$; la intensità $I$ in unità arbitrarie (secondo Vegard e Krogness).

per $\mathrm{cm}^{3}$ e per sec è dato, in funzione della quota, dalla espressione:

$$
L=L^{\prime} e^{-1-z-e^{-z}}
$$

essendo $z=\frac{h-h^{\prime}}{H}$, con $h=$ altezza rispetto al suolo, $h^{\prime}=$ altezza a cui $L(h)$ è massima e assume il valore $L^{\prime}, H=$ scala delle altezze.

(*) Si noti che i risultati di Lenard sono stati successivamente applicati da Chapman allo studio del processo di formazione degli strati ionizzati costituenti nel complesso la ionosfera. 
Quanto alla altezza $h^{\prime}$ essa è funzione dell'angolo di incidenza $\chi$ del fascio di particelle rispetto alla verticale, e la sua dipendenza da tale angolo $\chi$ è espressa dalla relazione

$$
h^{\prime}(\chi)-h^{\prime}(o)=H \ln \sec \chi .
$$

Poichè in pratica, si può ritenere, almeno nelle forme raggiate, che le particelle incidenti seguano le linee di forza del campo magnetico terrestre, descrivendo intorno ad esse un percorso elicoidale, la [2.2] è stata usata per valutare la differenza tra le quote di massima luminosità a latitudini diverse. $\mathrm{E}$ così pure, d'altra parte, dalla forma della curva di luminosità si sono assunte informazioni sulla composizione e sulla temperatura dell'alta atmosfera, dalle quali è condizionata la scala delle altezze $H$.

La fig. 2.13 mostra l'andamento della percentuale di energia assorbita e della luminosità al variare della quota per elettroni di diversa energia penetranti nella alta atmosfera in direzione delle linee di forza geomagnetiche: appare da tale figura che l'energia cinetica primaria degh elettroni che danno una luminosità massimla intorno ai $100 \mathrm{~km}$ dal suolo è di circa $30 \mathrm{keV}$. Con riferimento alla fig. 2.7 a l'abbassamento medio del bordo inferiore degli archi corrisponderebbe a valori crescenti della energia primaria, approssimativamente 7, 12, 22 e $30 \mathrm{keV}$ per aurore

Tabella 2.2.

\begin{tabular}{|c|c|c|c|c|c|c|}
\hline$l_{l}^{1 / 3}$ & $l^{1 / 2}$ & $l_{l}^{2 / 3}$ & $h_{\max }$ & $l_{u t}^{2 / 3}$ & $l_{u t}^{1 / 2}$ & $l_{u}^{1 / 3}$ \\
\hline 8.2 & 6.8 & 5.3 & 100 & 9.2 & 13.5 & 18.6 \\
\hline 8.8 & 7.2 & 5.7 & 110 & 10.3 & 15.2 & 20.6 \\
\hline 10.1 & 8.8 & 6.7 & 120 & 12.1 & 18.4 & 24.7 \\
\hline 12.3 & 11.0 & 8.2 & 130 & 14.3 & 22.0 & 29.3 \\
\hline 15.3 & 13.4 & 10.3 & 140 & 17.2 & 25.5 & 33.8 \\
\hline 22.7 & 18.0 & 13.8 & 150 & 21.8 & 30.8 & 41.2 \\
\hline 35.0 & 27.6 & 19.6 & 160 & $(33.0)$ & $(46.0)$ & $(60.0)$ \\
\hline 62 & 53 & 43 & 185 & 46 & 61 & 79 \\
\hline 135 & 105 & 76 & 270 & 87 & 117 & 146 \\
\hline
\end{tabular}




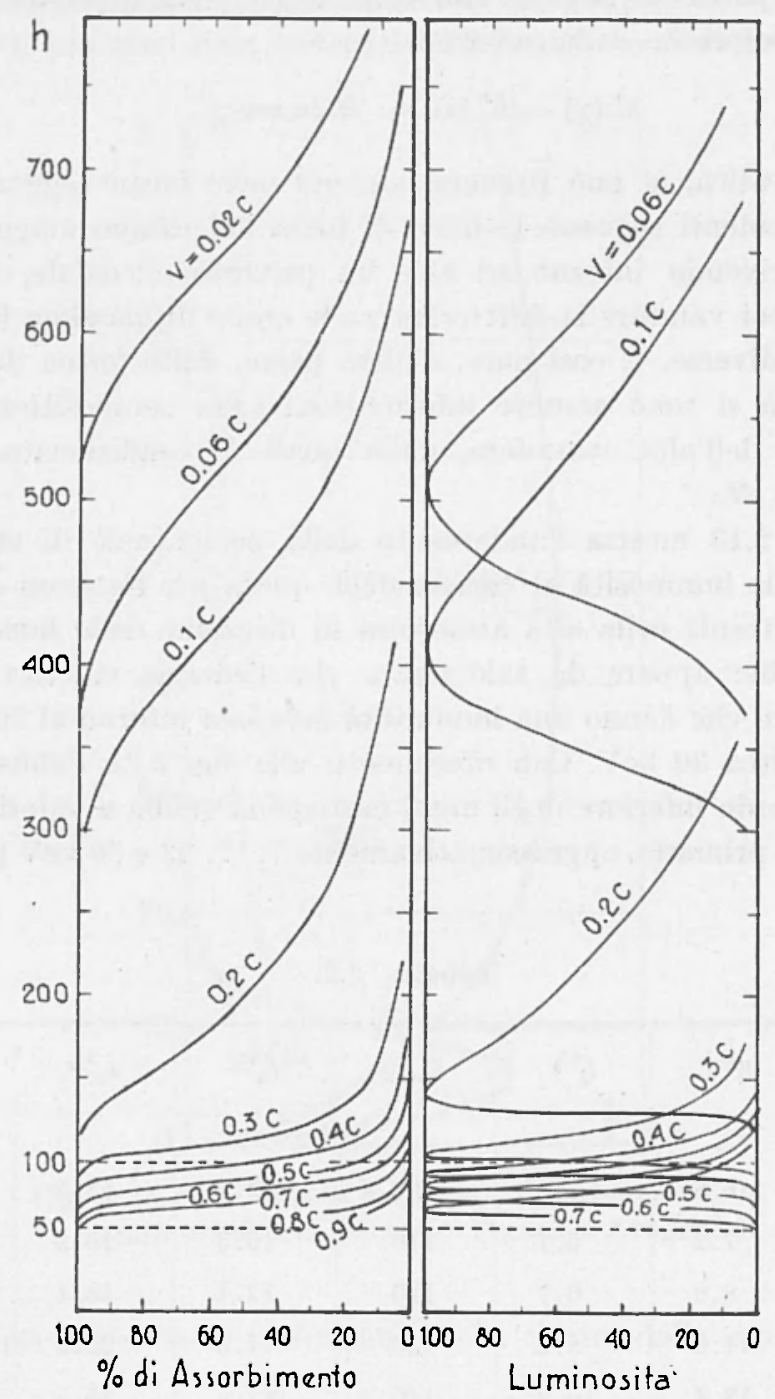

Fig. 2.13. - L'altezza $h$ è in $\mathrm{km}$; la luminosità è posta uguale a 100 in corrispondenza del suo massimo. Su ciascuna curva è riportata la velocità dei corrispondenti elettroni, in unità di velocità della luce $c$ (secondo Harang). 
deboli, medie, intense e molto intense. Per aurore il cui bordo inferiore raggiunge i $60 \div 70 \mathrm{~km}$, poi, l'energia cinetica primaria corrispondente sarebbe di $200 \div 300 \mathrm{keV}$.

La eventualità che le particelle che provocano l'aurora siano invece ioni positivi fu studiata da Vegard (44). In virtù del meccanismo di assorbimento delle particelle positive, la intensità di ionizzazione da queste prodotta è massima poco prima che esse vengano ridotte alla quiete e la corrispondente curva di luminosità mostra una rapidissima diminuzione dal suo valore massimo a zero nello spazio di solo qualche $\mathrm{km}$. In realtà l'evidenza sperimentale mostra che, pure essendoci casi di assai rapido decadimento di luminosità al bordo inferiore delle aurore, tuttavia, nella maggior parte di esse, la luminosità diminuisce più lentamente, secondo quanto risulta dalle curve di fig. 2.12 e dalla tabella 2.2 .

L'osservazione delle dimensioni trasversali dei raggi che costituiscono le forme raggiate consente di porre dei limiti alla energia massima delle particelle da cui essi sono prodotti: infatti se $E_{0}, T$, e sono rispettivamente l'energia di quiete $m_{0} c^{2}$. l'energia cinetica e la carica della singola particella, $F$ l'intensità del campo magnetico terrestre, $d / 2$ il raggio di curvatura della traiettoria, che non puo eccedere il semidiametro $d / 2$ della sezione di un raggio aurorale, vale, in unità di Gauss, la relazione

$$
T \leqslant-E_{0}+\left[E_{0}{ }^{2}+(e F d / 2)^{2}\right]^{1 / 2} \text {. }
$$

Ponendo $F=0,5$ oersted, $d=4 \cdot 10^{1} \mathrm{~cm}$, si ottengono, rispettivamente per elettroni e protoni, energie cinetiche $T=2,53 \mathrm{MeV}$ e $T=48 \mathrm{keV}$.

Sembra quindi di poter concludere che i raggi non possono essere attribuiti a protoni, in quanto questi avrebbero energia assai più bassa di quella minima loro necessaria per raggiungere le quote aurorali; in effetti, anche assumendo un diametro 10 volte maggiore, $d=4 \cdot 10^{5} \mathrm{~cm}$, si otterrebbe per l'energia $T$ dei protoni un valore $T=480 \mathrm{keV}$, ancora troppo basso.

2.4.2.1. - In un recente lavoro, Bates e Griffing ( ${ }^{45}$ ) hanno contestato la applicabilità delle [2.1] e [2.2], in quanto la diminuzione esponenziale del flusso di particelle al variare della massa d'aria attraversata, su cui si basa la loro deduzione, non è applicabile al caso specifico: infatti la legge esponenziale si applica al caso in cui la singola particella del fascio incidente che entra in collisione (elastica o anelastica) viene considerata perduta dal fascio stesso. In realtà, nel caso delle aurore anche le particelle "perdute" dal fascio incidente danno un contributo alla luminosità attraverso non a uno solo, bensì a tutta una serie di atti 
di eccitazione e ionizzazione delle particelle urtate. Altra obiezione alla applicabilità rigorosa dei risultati di Lenard sta nel fatto che la sezione d'urto per collisione degli elettroni non è costante al variare della loro velocità, ma cresce rapidamente allorchè la velocità stessa diminuisce $\left({ }^{46}\right)$.

2.4.3. Colore e spettro. - Il colore delle aurore varia ampiamente da aurora a aurora, oltrechè nel corso di una stessa apparizione aurorale. Il colore più comune che si osserva negli archi, nelle bande e nei raggi, sopratutto negli anni di bassa attività solare, è il giallo-verde. Meno comune è il rosso che, di solito limitato alla parte più alta dei raggi e talvolta alle superfici diffuse (aurore rosse di tipo A) ovvero alla parte inferiore degli archi, a mo' di bordo rosso (aurore rosse di tipo B), appare in alcuni casi estendersi dalla base alla sommità dell'aurora, quale ne sia il tipo. Altre colorazioni osservate in numerosi casi sono l'arancio, durante intense aurore con lunghi raggi e corona, il violetto, il bianco, e assai raro, 11 bleu.

La grande varietà di colorazione delle aurore, anche se con prevalenza di certi colori principali, fa intuire una complessa composizione spettrale delia luce aurorale; l'interpretazione degli spettrogrammi è resa più difficile dalla sovrapposizione, con intensità assai variabili, $d$ spettri di righe, propri degli atomi, e spettri di bande, propri delle molecole (tav. IX e X).

L'esame critico delle osservazioni sperimentali, intrapreso solo da pochi anni, consente però di trarre notevoli risultati; tuttavia, poichè una trattazione completa della spettroscopia delle aurore esce dai limiti del nostro lavoro, ci limitiamo a considerare principalmente quei risultati che hanno più stretta connessione col problema della determinazione del tipo di agente (primario o no) che eccita la luminosità aurorale e le sue caratteristiche fisiche (energia, densità di flusso, ecc.).

2.4.3.1. Le righe dell'ossigeno e dell'azoto atomici. - Sono presenti righe dovute sia a transizioni permesse che a transizioni proibite. In particolare sono ben evidenti le righe dell'ossigeno e dell'azoto atomici, non ionizzati, $O I$ e $N I$; le righe dell $O I I$ e $N I I$ sono invece molto deboli e non si può ancora ritenere del tutto certa la loro completa identificazione. Dubbia infine appare la presenza di righe proibite dell'OIII e dell' $N I I I$.

Secondo Vegard (47) il numero di righe dell'OI, OII, OIII, NI, $N I I, N I I I$ che dovrebbero essere con maggiore probabilità presenti nello spettro dell'aurora sarebbe rispettivamente di 59, 128, 8, 106, 81, 24 . 
Tra le dette righe atomiche le più intense e caratteristiche sono le tre righe dell' $O F \quad \lambda 5577,35 \AA$, la cosidetta " riga aurorale " dovuta alla transizione proibita ${ }^{1} S_{0}-{ }^{1} D_{2}, \lambda 6300,3 \AA$, dovuta alla transizione proibita ${ }^{1} D_{2}-{ }^{3} P_{2}$, e $\lambda 6363,8 \AA$, dovuta alla transizione proibita ${ }^{1} D_{2}-{ }^{3} P_{1}$.

2.4.3.2. Le righe dell'idrogeno e dell'elio atomici. — La presenza occasionale delle righe $H_{\alpha}$ e $H_{\beta}$ dell'idrogeno, appartenenti alla serie di Balmer, fu riscontrata per la prima volta da Vegard ${\left({ }^{\mathbf{4 8 , 4 9}}\right)}$ nella parte inferiore di archi omogenei quieti, il 18 ottobre 1939. E poi risultata presente anche la riga $H_{\gamma}$; le lunghezze d'onda corrispondenti alle righe $H_{\alpha}, H_{\beta}, H_{\gamma}$ sono rispettivamente $\lambda=6563,4861$ e $4341 \AA$. La presenza delle righe di Balmer ha notevole interesse per la conoscenza del tipo di particelle incidenti sull'atmosfera in determinate fasi del fenomeno aurorale. Già Vegard, fin dalla prima occasione in cui furono osservate le righe $H_{\alpha}$ e $H_{\beta}$, notava che nelle parti alte dei raggi solo assai raramente si rilevava la presenza di idrogeno e ne concludeva che la presenza delle righe dell'idrogeno doveva essere attribuita a sciami di atomi di idrogenu o a un tipo di "radiazione di idrogeno ", occasionalmente emessi dal Sole ed entranti nella atmosfera durante le apparizioni aurorali (riprenderemo questa questione in 2.4.4.1).

Quanto all'elio, fino a questo momento non risulta nè accertata nè esclusa in maniera conclusiva la presenza delle sue righe caratteristiche; in effetti, alcune deboli righe aurorali sono state attribuite da Bernard ( $\left.{ }^{50}\right)$ all' $H e I$ e all' $H e I I$; la presenza di elio resta tuttavia dubbia sopratutto per la mancanza negli spettri aurorali di talune righe che dovrebbero risultare abbastanza bene individuabili (v. 2.4.4.3).

2.4.3.3. Le bande dell'azoto $e$ dell'ossigeno molecolari. - Le bande più intense sono quelle dell'azoto neutro e dell'azoto una volta ionizzato; quelle dell'ossigeno sono invece piuttosto deboli, fatta eccezione per le aurore che compaiono a quote inferiori ai $100 \mathrm{~km}$, in quanto l'ossigeno verso i $100 \mathrm{~km}$ è ormai quasi completamente dissociato.

Sono presenti le seguenti bande dell'azoto: a) primo gruppo positivo dell' $N_{2}$, nell'infrarosso e nel rosso; $b$ ) secondo gruppo positivo dell' $N_{2}$, nell'ultravioletto, le cui bande più intense (più prossime al visibile) sono situate intorno alle lunghezze d'onda 4059 e $3997 \AA$; $c$ ) il gruppo di Vegard-Kaplan dell' $N_{2}$, le cui bande si estendono nel violetto e nell'ultravioletto; $d$ ) il primo gruppo negativo dell' $N_{\Delta}^{+}$, le cui bande più intense si hanno intorno alle lunghezze d'onda 3914, 4278 e $4708 \AA$ : e) il gruppo di Meinel o secondo gruppo negativo dell' $N_{4}^{+}$, principalmente presente nell'infrarosso, osservato da Meinel ( ${ }^{(51)}$ e rivelatosi di partico- 
lare interesse nelle teorie della eccitazione della luminosità aurorale (v. 2.4.4.2).

Per ciò che concerne l'ossigeno, le bande più notevoli sono raggruppate nel gruppo di Schumann-Runge dell' $O_{2}$, nell'infrarosso, e nel primo gruppo negativo dell' $\mathrm{O}_{2}^{+}$, nella zona del verde.

2.4.3.4. Variazioni nel colore e nello spettro delle aurore. - Lo studio sistematico di tali variazioni, sopratutto ad opera di Vegard, ha messo in evidenza numerose particolarità di cui qui ricosdiamo brevemente solo le più importanti.

Si hanno:

a) Variazioni con l'altezza. - Nella parte verde-azzurra dello spettro, la intensità della riga $\lambda 5577$ negli archi decresce con la quota riducendosi $\left({ }^{46}\right)$ nelle parti più alte a circa $75 \%$ di quella al bordo inferiore; quanto alle bande violette del primo gruppo negativo dell' $N_{2}^{+}$, la loro intensità relativa a quella della riga verde tende a crescere con la quota. Piuttosto irregolari risultano invece le variazioni di intensità per le altre forme aurorali. Nella parte dello spettro verso il rosso la intensità delle righe rosse dell'OI, sempre riferita alla intensità della riga verde, cresce dal bordo inferiore al limite superiore dell'aurora, dal $1 \pm 0$ al $300 \%$ a seconda del tipo aurorale considerato. Le bande rosse del primo gruppo positivo dell' $N_{2}$ risultano invece più intense al bordo inferiore, al contrario di quanto avviene per le bande violette.

b) Variazioni con la latitudine. - L'intensità delle righe rosse $\lambda 6300$ e $\lambda 6364$ e quella della riga verde $\lambda 5577$ crescono al diminuire della latitudine $\left({ }^{49}\right)$; e cosi pure crescono le intensità delle righe dell'idrogeno, e, probabilmente, delle bande del gruppo di Vegard-Kaplan dell' $N_{2}$.

c) Variazioni con la attività solare. - Le intensità medie delle righe $\lambda 6300$ e $\lambda 6364$ dell $O I$, riferite a quelle della riga verde, appaiono maggiori nei periodi di alta attività solare. Come si è già accennato, le aurore rosse sono state distinte ( $\left.{ }^{47}\right)$ in due tipi, A e B, dei quali il primo è più frequente nei periodi di alta attività solare e il secondo, invece, sembra essere più frequente nei periodi di minima attività solare: la distinzione nei due tipi è determinata dal fatto che le aurore di tipo $\mathbf{A}$ debbono principalmente il loro colore alla riga $\lambda 6300$ dell' $O I$, mentre quelle di tipo B lo debbono ad una accresciuta intensità delle bande del primo gruppo positivo dell' $N_{2}$.

d) Altri tipi di variazioni. - In generale si riscontra, nelle aurore deboli e diffuse, una intensità relativa delle bande violette del pruno 


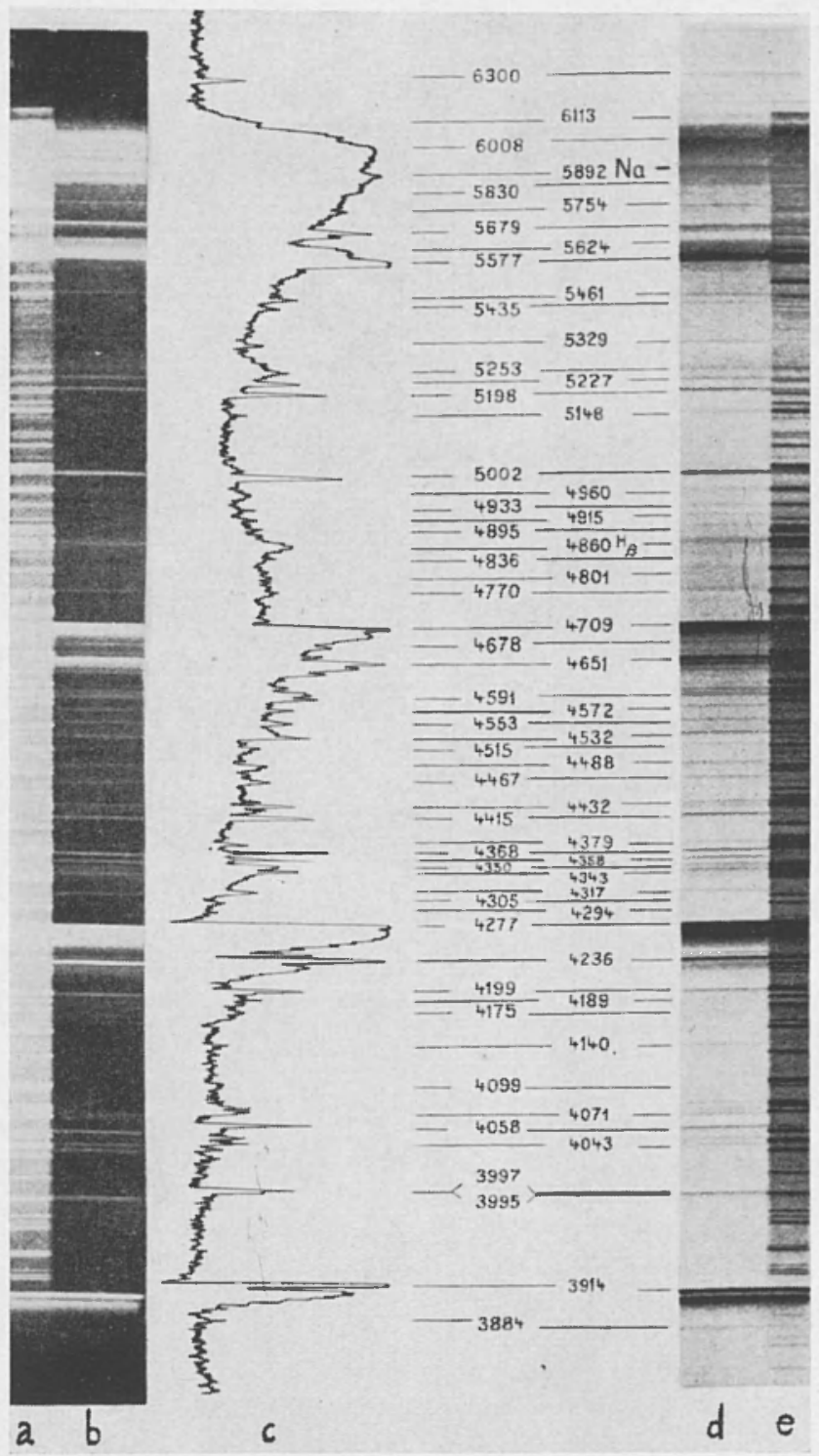

Spettrogramma ottenuto da Vegard e Kvifte, a Oslo, la notte del 23 febbraio 1950. Da sinistra verso destra: a spettro di riferimento fornito da una lampada ad argon; $b, c$ spettro aurorale e corrispondente registrazione fotometrica; $d, e$ immagine negativa degli spettri $b$ e $a$, rispettivamente. Sono indicate le lunghezze d'onda in $A$. 

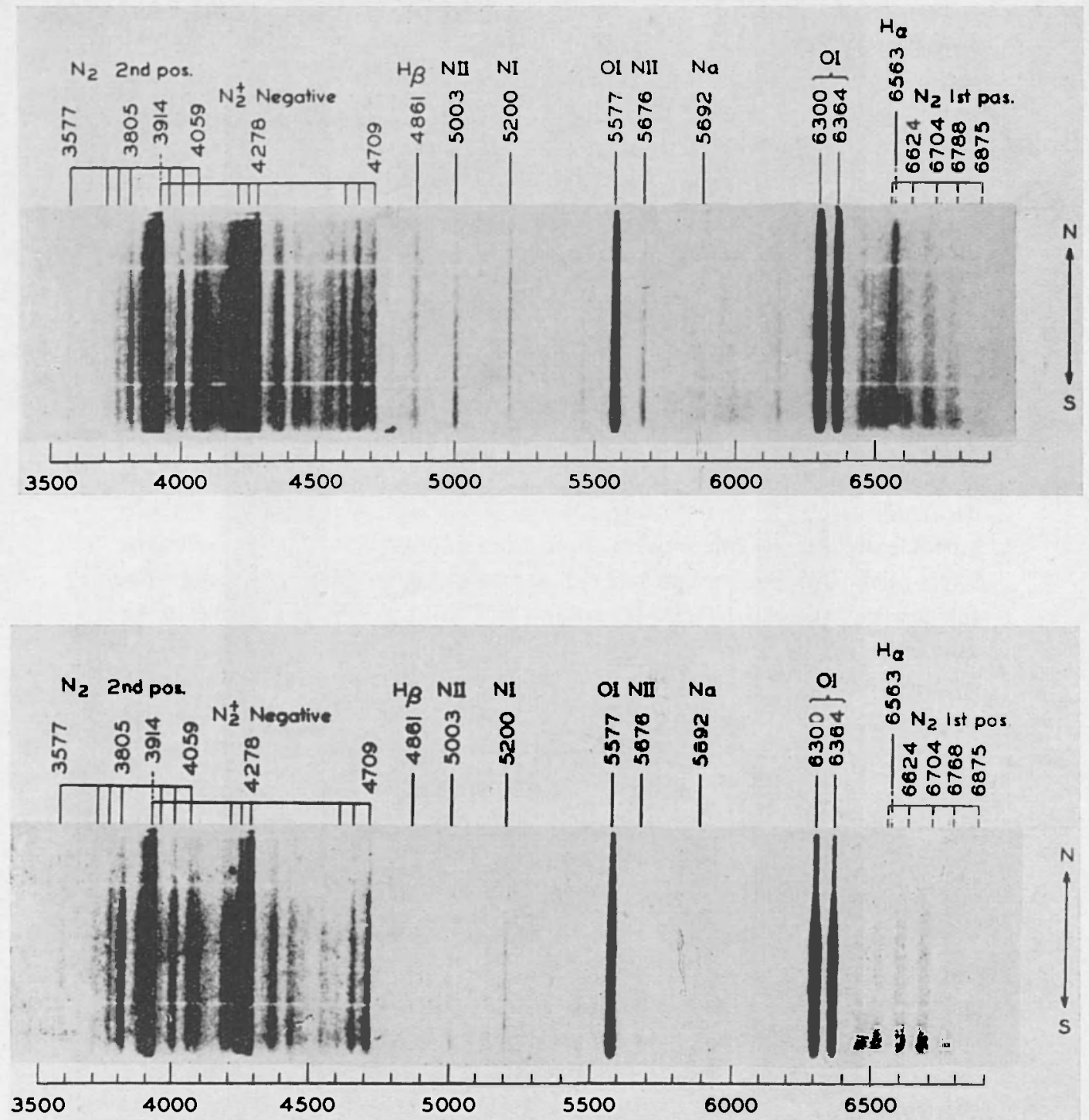

Due spettri tipici di aurore ottenuti nella direzione del meridiano geomagnetico; nel primo ̀̀ ben evidente una intensa emissione $H_{\alpha}$ e $H_{\beta}$ dell'idrogeno, che è del tutto assente nel secondo (per cortesia del Geophysical Institute University of Alaska, College). 


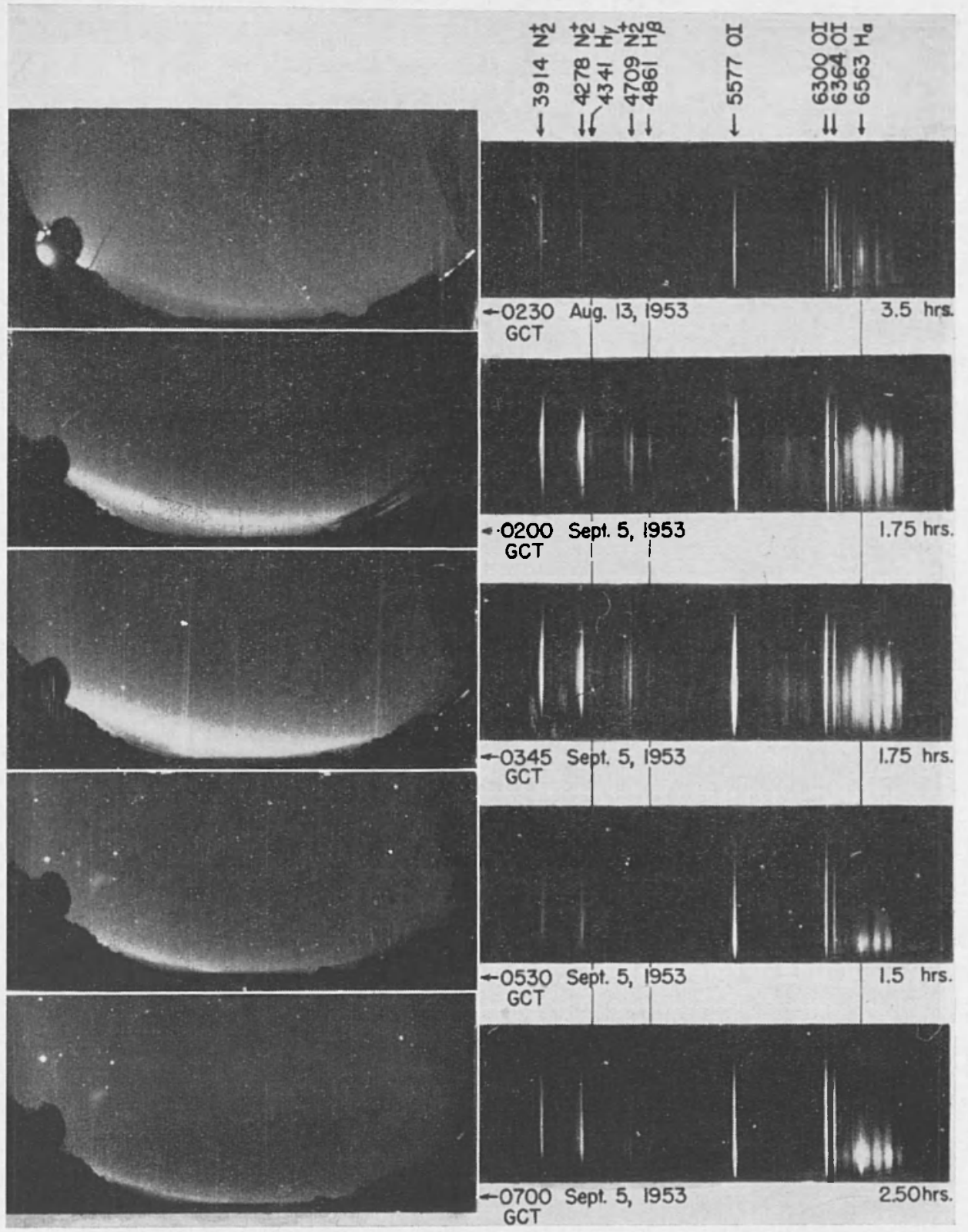

Sequenza di spettri aurorali osservati a Yerkes il 13 agosto e il 5 settembre 1953; in corrispondenza a ogni spettro il tempo di posa e una fotografia dell'aurora (arco omogeneo) presa durante la posa stessa (per cortesia dei dr. C.Y. Fan e D. H. Schulte dello Yerkes (Observatory, Chicago). 


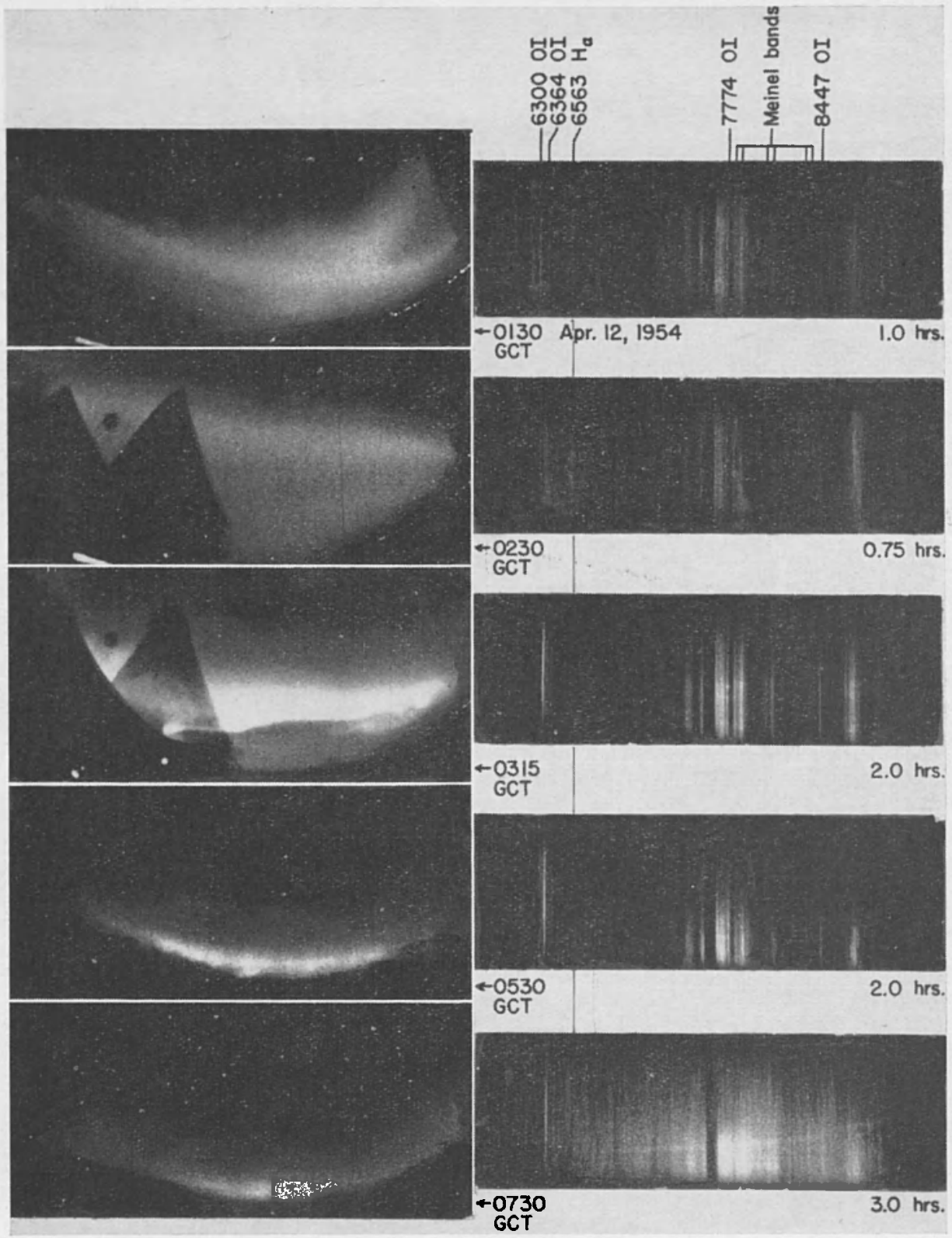

Sequenza di spettri aurorali osservati a Yerkes il 12 aprile 1954. Molto bruscamente larco omogeneo si trasforma in raggi brillanti e scompare decisamente ogni traccia della riga $H_{\alpha}$ (per cortesia dei dr. (C. Y. Fan e D. H. Schulte, Chicago). 
gruppo negativo dell' $\mathrm{N}_{2}{ }^{+}$rispetto a quella della riga verde, maggiore che nelle forme aurorali più intense. Se si ricorda, come si è visto in 2.2.3, che le forme aurorali intense sono in media più basse di quelle deboli, tale effetto risulta evidentemente, almeno in parte, un effetto di altezza, appunto in accordo con quanto si è detto in a), a proposito delle bande violette.

Non infrequenti, specialmente nelle aurore più luminose, sono pure improvvise variazioni di colore, per es. da verde a rosso, senza alcuna concomitante variazione di posizione o di estensione della forma aurorale.

2.4.4. La natura dell'agente cui sono dovute le aurore. - Lo studio delle diverse caratteristiche degli spettri aurorali, in particolare delle righe dell'idrogeno, consente di ottenere importanti risultati sulla natura delle particelle primarie che eccitano la luminosità aurorale, sul meccanismo di accelerazione, sulla densità di flusso e sulla energia.

2.4.4.1. - Nel 1950, Vegard $\left({ }^{52,}{ }^{53}\right)$ segnalava uno spostamento verso il violetto nella riga $H \beta$, attribuendolo al moto verso il basso di atomi di idrogeno.

Gartlein ( $\left.{ }^{54}\right)$ metteva in evidenza che le intensità relative delle tre righe $H_{\alpha}, H_{\beta}$, e $H_{\gamma}$ restano le stesse in un vasto campo di variabilità delle rispettive intensità assolute, indipendentemente dal comportamento delle altre righe; inoltre, puntando lo spettrografo verso lo zenith geomagnetico e verso l'orizzonte geomagnetico, Meinel $\left({ }^{55,56}\right)$ osservava (fig. 2.14), in occasione delle aurore del 18 e 19 Agosto 1950 studiate all'Osservatorio di Yerkes, essere il profilo della riga asimmetrico nel primo caso e simmetrico invece, sebbene allargato, nel secondo caso, ciò che è ancora indice di un moto di insieme di atomi di idrogeno verso il basso. Nella parte inferiore della stessa fig. è riportato lo spettrogramma ottenuto, nella stessa occasione, dopo la trasformazione dell'areo omogeneo in una forma fiammeggiante.

In tutte le osservazioni fatte, lo spostamento del massimo di intensità delle righe dell'idrogeno verso il violetto, per effetto Döppler, è dell'ordine di parecchi $\AA$; in particolare, Meinel, correggendo gli spettrogrammi di fig. 2.14 per la presenza delle bande del primo gruppo positivo dell' $N_{2}$ sottraendo ad essi lo spettrogramma ottenuto in assenza di emissione $H_{\alpha}$, ha ottenuto (fig. 2.15) uno spostamento massimo verso il violetto della riga $H_{\alpha}$ di $71 \AA$, corrispondente a uma velocità di penetrazione nella atmosfera di circa $3300 \mathrm{~km} / \mathrm{sec}$. Numerose osserva- 
zioni $\left({ }^{57}, 58,{ }^{59}\right)$ hanno mostrato essere le righe dell'idrogeno assai intense negli archi omogenei e molto deboli o assenti, invece, nelle forme raggiate (tav. XI e XII). Peraltro ne sono state osservate notevoli variazioni di intensità su fotografie prese in rapida successione $\left({ }^{60,}{ }^{61}\right)$.

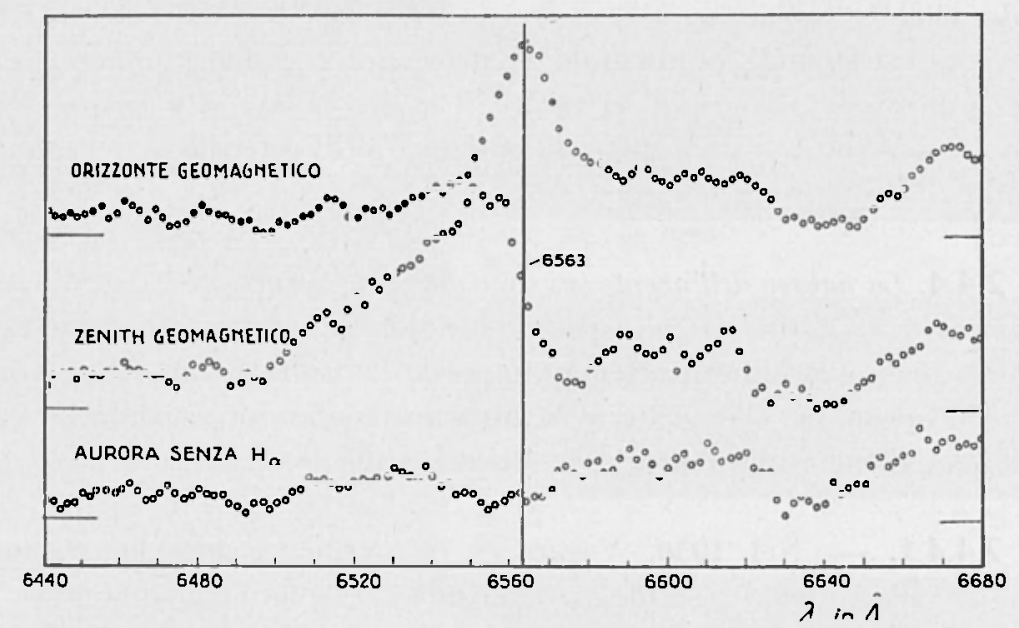

Fig. 2.14. - Analisi microfotometrica di spettro aurorale in prossimità della riga $H_{\alpha}$ (secondo Meinel).

Ad un primo esame la velocità di $3300 \mathrm{~km} / \mathrm{sec}$ trovata da Meinel sembra da considerarsi come la velocità di incidenza di una radiazione protonica, uguale per tutte le particelle, entrante nell'alta atmosfera; lo sparpagliamento delle velocità che risulta dalla fig. 2.15 andrebbe attribuito al frenamento dei corpuscoli incidenti, man mano che essi penetrano nella atmosfera. Occorre tuttavia considerare che la probabilità che si abbia emissione di radiazione $H_{\alpha}$ in conseguenza della cattura di elettroni da parte di protoni è massima ( ${ }^{62}$ ) allorchè i protoni hanno una velocità di circa $730 \mathrm{~km} / \mathrm{sec}$ (che è la velocità orbitale di Bohr per il livello $3 \mathrm{~s}$ ) e decresce rapidamente al crescere della velocità.

Ne consegue, allora, che non si può escludere nel fascio di particelle primarie la presenza di protoni di velocità maggiore di $3300 \mathrm{~km} / \mathrm{sec}$, che non risultano però dalle misurazioni sull'effetto Doppler in quanto solo alle basse velocità è apprezzabile la probabilità di emissione di radiazione $H_{\alpha}$.

La velocità dedotta da Meinel è notevolmente maggiore di quella, non superiore ai $1000 \mathrm{~km} / \mathrm{sec}$, che Richardson $\left({ }^{63}\right)$ e Brïck e Rutllant ( ${ }^{64}$ ) 
hanno attribuito alle particelle emesse dal Sole, secondo misure di effetto Döppler nella regione delle righe $H(\lambda 3968,5)$ e $K(\lambda 3933, \tau)$ del CaII dello spettro solare, in occasione di tempeste magnetiche.

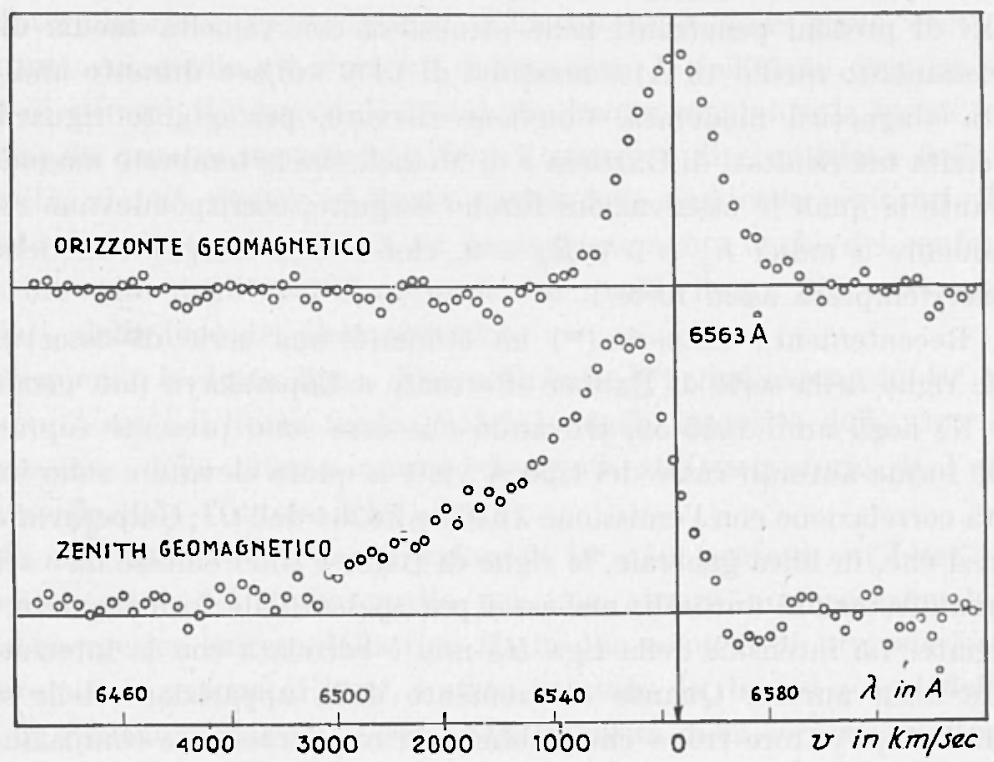

Fig. 2.15. - Curve corrette corrispondenti a quelle di fig. 2.14; sulle ascisse è riportata anche la scala delle velocità di penetrazione (secondo Meinel).

In effetti, se si assume un intervallo di tempo di $1 \div 2$ giorni tra il fenomeno solare cui si attribuisce una tempesta magnetica e il manifestarsi di questa sulla Terra, la velocità media di propagazione delle particelle primarie risulta dell'ordine dei $1000 \div 2000 \mathrm{~km} / \mathrm{sec}$ per traiettoria Sole-Terra rettilinea. Tuttavia è piuttosto probabile che la effettiva traiettoria delle particelle primarie sia più o meno sensibilmente incurvata, il che può determinare allungamenti anche notevoli di percorso; le elevate velocità misurate da Meinel potrebbero poi essere semplicemente dovute ad una accelerazione dei protoni primari da parte di un campo elettrico presente in prossimità della Terra.

In conclusione, tenendo conto dell'incurvamento delle traiettorie, le suddette velocità di $1000 \div 2000 \mathrm{~km} / \mathrm{sec}$ andrebbero alquanto aumentate; d'altra parte, l'effetto di una accelerazione "locale" dei protoni potrebbe essere quello di aumentare la loro velocità, inizialmente molto più bassa, soltanto nella ultima parte della traiettoria. La correzione 
apportata da questi due effetti può allora plausibilmente essere tale da portare alla riduzione, se non addirittura alla eliminazione, delle discrepanze tra le velocità dei protoni sopra riscontrate.

Anche Gartlein ( $\left.{ }^{65}\right)$, poco tempo dopo Meinel, ha constatato la presenza di protoni penetranti nella atmosfera con velocità media di 675 (spostamento medio $15 \AA$ ) e massima di $1350 \mathrm{~km} / \mathrm{sec}$ durante una tempesta magnetica moderata. Conviene rilevare, per quanto riguarda la diversità nei risultati di Gartlein e di Meinel, che le tempeste magnetiche durante le quali le osservazioni furono eseguite, corrispondevano rispettivamente a indici $K_{p}=5$ e $K_{p}=9$, ciò̀ a una tempesta moderata e a una tempesta assai forte.

Recentemente Galperin $\left(^{68}\right)$ ha studiato una serie di osservazioni delle righe della serie di Balmer effettuate a Loparskaya (lat. geomagn. $64^{\circ} \mathrm{N}$ ) negli anni $1955-58$, trovando che esse sono presenti sopratutto nelle forme aurorali rosse del tipo $\Lambda$ viste a quote elevate e sono in una certa correlazione con l'emissione $\lambda .6300$ e $\lambda .6364$ dell' $O I$; Galperin afferma altresi che, in linea generale, le righe di Balmer sono emesse da vari tipi di manifestazioni aurorali, ma assai più spesso dalle forme quiete, non raggiate. La intensità della riga $H_{\alpha}$ non è correlata con la intensità visuale della aurora. Quanto al momento della apparizione delle righe di Balmer, l'autore trova che, sebbene in molti casi esse compaiano simultaneamente all'aurora visuale, tuttavia si hanno anche casi in cui esse compaiono una o due ore prima.

Anche dai risultati di Galperin appare chiaramente l'effetto Doppler; tuttavia, in media, lo spostamento verso il violetto corrisponde a velocità medie di qualche centinaio di $\mathrm{km} / \mathrm{sec}$ e massime di circa $2000 \mathrm{~km} / \mathrm{sec}$, valori più elevati risultando eccezionali; appare anche un lieve allargamento verso il rosso del profilo delle righe, corrispondente a una velocità massima di $300 \div 500 \mathrm{~km} / \mathrm{sec}$. La intensità relativa delle righe $H_{\beta}$ e $H_{\gamma}$ è 1:0,80.

Altro aspetto interessante è che, mettendo in relazione tra loro la apparizione e la scomparsa della riga $H_{a}$ con la radioaurora, è risultato che nel $65 \%$ dei casi la $H_{\alpha}$ è apparsa e scomparsa insieme al radioeco; nel $26 \%$ dei casi la $H_{\alpha}$ è apparsa senza che si avesse radioeco; nel $9 \%$ di casi, infine, si sono avuti radioechi senza osservazione della $H_{\alpha}$.

Questi ultimi risultati forniscono ulteriore sostegno agli argomenti tratti dalle osservazioni di effetti Doppler, in favore dell'arrivo di una radiazione corpuscolare dall'esterno durante le aurore.

In conclusione, le osservazioni sperimentali concordemente indicano la presenza delle righe di Balmer negli spettri aurorali propri delle 
forme quiete, degli archi in particolare; la eccitazione di tali righe è attribuibile a fasci di protoni che dallo spazio esterno incidono sulla atmosfera.

2.4.4.2. La densità di flusso e l'energia delle particelle primarie. La determinazione spettrografica della intensità delle righe spettrali, sopratutto di quelle più evidenti dell'ossigeno e dell'azoto atomici, consente di stimare il numero di fotoni che hanno raggiunto la lastra fotografica; da questo, supponendo noto il processo di eccitazione della luminosità, si può risalire al flusso medio delle particelle eccitanti. Tale metodo di indagine si giova assai vantaggiosamente anche del confronto delle intensità degli spettri aurorali con quelli, finora più estesamente studiati, della luce del cielo notturno.

Seguendo le dette linee, Fan e Schulte $\left(^{59}\right)$ giudicarono in $10^{7}$ protoni $\cdot \mathrm{cm}^{-2} \cdot \mathrm{sec}^{-1}$ il flusso medio, valutato dalla intensità della riga $H_{\alpha}$, in occasione della intensa aurora osservata all'Osservatorio di Yerkes il 12 aprile 1954 (Tavola XII).

Si può ritenere $\left.{ }^{(87}\right)$ che il valore di $10^{7} \div 10^{8}$ protoni $\cdot \mathrm{cm}^{-2} \cdot \mathrm{sec}^{-1}$ sia rappresentativo del flusso medio per una aurora non eccezionale. Il flusso energetico emesso dalla riga $H_{\alpha}$ in una colonna di atmosfera sede di aurora e di sezione di $1 \mathrm{~m}^{2}$, è stato valutato da diversi autori dell'ordine di qualche $\mathrm{erg} \cdot \mathrm{sterad}^{-1} \cdot \mathrm{sec}^{-1}$. La intensità della corrente protonica primaria, cui per ragioni di equilibrio elettrostatico va associata una pressochè identica corrente elettronica, è dell'ordine delle centinaia o delle migliaia di ampere; per es., dall'esame di numerosi spettrogrammi ottenuti a Ithaca, Gartlein e Sprague ( ${ }^{88}$ ) hanno stabilito in $10^{3}$ ampere l'ordine di grandezza della intensità di tale corrente.

Quanto alla energia cinetica $T$ delle particelle primarie, e allo spessore d'aria che sono capaci di attraversare, essi sono mostrati in fig. 2.16 a e $b$ per gli elettroni e per i protoni. Occorre tuttavia aggiungere che finchè non sia ben chiarito il meccanismo di eccitazione e di emissione della luminosità aurorale, i risultati mostrati nella figura non sono appllcabili, senza riserva, per dedurre l'energia delle particelle aurorali, in quanto la luminosità potrebbe essere in maniera anche rilevante controllata da altri fattori, oltrechè dallo spessore globale di materia attraversata. La relativa costanza nel tempo della quota del bordo inferiore di un arco potrebbe essere un argomento in favore di questa riserva; infatti, se si ammette, come è plausibile, che le energie dei protoni primari siano contenute in uno spettro piuttosto esteso, allora i soli effetti sulla cui considerazione si basa la fig. 2.16 non renderebbero ragione di un bordo inferiore cosi ben definito, come in effetti si osserva. 


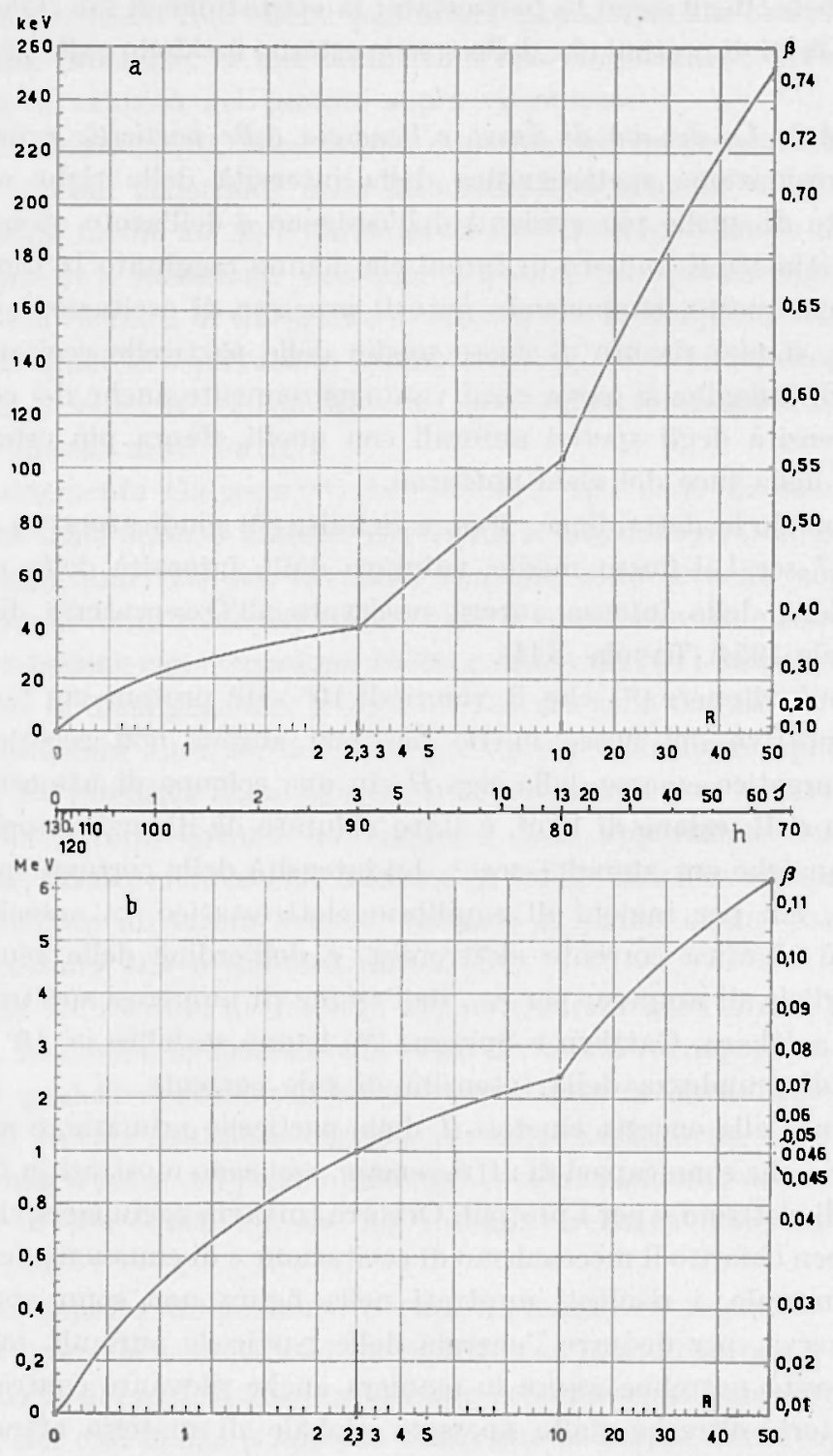

Fig. 2.16. - Relazione range-energia: $a$ per elettroni; $b$ per protoni. Il range $R$ è in cn di aria a $15{ }^{\circ} \mathrm{C}$ e $760 \mathrm{mmHg}$; lo spessore $s$ in $\mathrm{mg} / \mathrm{cm}^{2}$; l'altezza $h$ dal suolo a cui penetrano le particelle è in $\mathrm{km}$. La scala di destra da il rapporto $\beta=v / c$. I punti sulle curve corrispondono ai cambiamenti di unità sulle scale. 
2.4.4.3. Esperimenti di laboratorio e loro contributo alle teorie aurorali. - Le ricerche iniziate da Meinel dopo il 1951 nell'intento di investigare i processi fondamentali che danno origine all'aurora sono state notevolmente estese da Meinel e Fan. Questi autori lianno studiato lo spettro di emissione di una massa d'aria a pressioni tra 0,1 e $0,5 \mathrm{~mm}_{\mathbf{H g}}$ sottoposta a bombardamento da parte di protoni, ioni $\mathrm{He}^{+}$, particelle $\alpha$ ed elettroni di energie rispettivanente comprese tra 5 e 350, 10 e 450, 75 e $320,0,01$ e $8 \mathrm{keV}$. Lo studio di tali spettri è notevolmente difficoltoso, in quanto l'emissione dovuta ad elettroni secondari e gli effetti di parete possono alterarne la struttura in modo notevole e non facilmente identificabile.

Le prime indagini di laboratorio furono condotte da Meinel e Fan ( ${ }^{68}$ ) con protoni aventi energia di $230 \mathrm{keV}$; essi ottennero uno spettro assai somigliante (tav. XIII) a quello aurorale; in particolare la intensità relative delle bande deli' $N_{2}^{+}$e dell' $N_{2}$ sono assai simili per lo spettro aurorale e per quello ottenuto in laboratorio; tuttavia alcune bande ultraviolette in prossimità dei $3500 \AA$ sono notevolmente più intense nell'aurora che in laboratorio.

Le successive indagini condotte con protoni e ioni $\mathrm{He}^{+}$hanno messo in evidenza $\left({ }^{70}\right)$ interessanti differenze nelle emissioni dell' $N I I$ e dell' $\mathrm{N}_{2}{ }^{+}$: il rapporto tra le loro intensità è sensibilmente indipendente dalla energia delie particelle incidenti, mentre invece è assai diverso a seconda della natura delle particelle stesse. In effetti il rapporto tra le intensità $I_{500 s}$ e $I_{5880}$ delle due righe $\lambda 5004$ e $\lambda 5680$ dell' $N I I$ rispetto a quella $I_{5288}$ della riga $\lambda 5228$ del secondn gruppo positivo dell' $N_{2}+$ risulta da 5 a 10 volte maggiore quando l'eccitazione è provocata da ioni $\mathrm{He}^{+}$anzichè da protoni: in particolare, il rapporto $I_{5004} / I_{5228}$ vale 1,2 per eccitazione protonica (a $150 \mathrm{keV}$ ) e 11,6 per eccitazione con ioni $\mathrm{He}^{+}$(a $150 \mathrm{keV}$ ). Il fatto che nella aurora tale rapporto risulta sensibilmente superiore a 1,2, suggerisce una eccitazione mista della luminosità aurorale, da parte sia di protoni che di ioni $\mathrm{He}^{+}$: gli autori, con tale metodo indiretto, giudicano in circa $10 \%$ l'abbondanza relativa delle ioni $\mathrm{He}^{t}$ rispetto ai protoni nel fascio di radiazione primaria. Tale abbondanza relativa dell'idrogeno e dell'elio risulta circa la stessa che si riscontra nelia composizione media dell'atmosfera solare, oltrechè nella composizione della radiazione cosmica primaria (v. capitolo 4).

Sulla questione delia eventuale presenza delle righe dell'elio nello spettro aurorale (tav. XIV) è tornato successivamente Fan ( $\left.{ }^{11},{ }^{22}\right)$ : nei primi esperimenti di laboratorio le righe non presentavano alcuno spostamento per effetto Döppler, come invece appariva per le righe dell'i- 
drogeno $\left({ }^{70}\right)$. In effetti risulta da esperienze condotte da Krasner $\left({ }^{73}\right)$, che ha determinato la sezione d'urto per il processo di perdita per collisione dell'elettrone ottico da parte di un atomo di $\mathrm{Fe}$, che nelle condizioni in cui gli spettri erano stati osservati da Fan la vita nedia di tale elettrone era sufficientemente breve perchè l'atomo di He ne venisse privato prima che fosse possibile la transizione ottica. Nuove osservazioni spettroscopiche condotte in condizioni più appropriate, a pressione di $10^{-2} \div 10^{-4} \mathrm{~mm}_{\mathrm{Hg}}$, con ioni $\mathrm{Ie}^{+}$di energia fino a $450 \mathrm{keV}$ hanno mostrato invece chiaramente l'effetto Doppler. Le caratteristiche proprie dello spettro eccitato dall' $\mathrm{He}^{\dagger}$ e le differenze rispetto a quello eccitato da protoni, sono tali da rendere possibile di apprezzare sperimentalmente un contenuto percentuale di ioni $\mathrm{He}^{+}$non inferiore a circa il $5 \%$; tuttavia, prima di uno studio in questo senso, sarebbe conveniente che venisse accertata o esclusa in maniera conclusiva la presenza delle righe caratteristiche dell'elio: le righe più facilmente individuabili dovrebbero essere la $\lambda .5876$ e la $\lambda 3188$ nell'ultravioletto; un'altra riga caratteristica sarebbe la $\lambda .6678$ che però, a differenza delle due precedenti, non è bene individuabile perchè cade in corrispondenza di una intensa banda del primo gruppo positivo dell' $N_{2}$, intorno alla lunghezza d'onda 2.6705 .

Per quanto concerne la zona infrarossa dello spettro, le bande di Meinel dell' $N_{2}^{+}$risultano, nella encitazione con protoni o ioni $\mathrm{He}^{+}$ di energia tra 40 e $400 \mathrm{keV}$, assai più deboli di quelle del primo gruppo positivo dell' $N_{2}$ osservato nello spettro aurorale. Ulteriori esperimenti di Fan $\left({ }^{74}\right)$ hanno mostrato che è possibile ottenere le bande di Meinel, con la intensità che esse hanno nello spettro aurorale, per eccitazione con elettroni di energia delle centinaia o delle migliaia di $\mathrm{eV}$. $\mathrm{A}$ questo proposito, occorre aggiungere che le più recenti ricerche sperimentali dello stesso autore $\left({ }^{75}\right)$ hanno messo in evidenza delle notevoli e insospettate difficoltà di interpretazione degli spettri ottenuti in laboratorio; Fan ritiene tuttavia che la interpretazione elettronica della eccitazione del gruppo di Meinel resti sostanzialmente corretta.

L'origine degli elettroni che eccitano le bande di Meinel merita di essere studiata più in dettaglio: se tali elettroni fossero di origine primaria, allora essi per raggiungere una data quota dovrebbero possedere velocità assai maggiori di quelle che si richiedono ai protoni per giungere alla stessa quota. In tal caso lo spettro aurorale risulterebbe eccitato da un fascio misto di elettroni veloci e protoni lenti; appoggiandosi su taluni risultati deducibili dalla teoria di Alfven delle tempeste magnetiche, peraltro non da tutti accettata, Fan osserva che il moto 


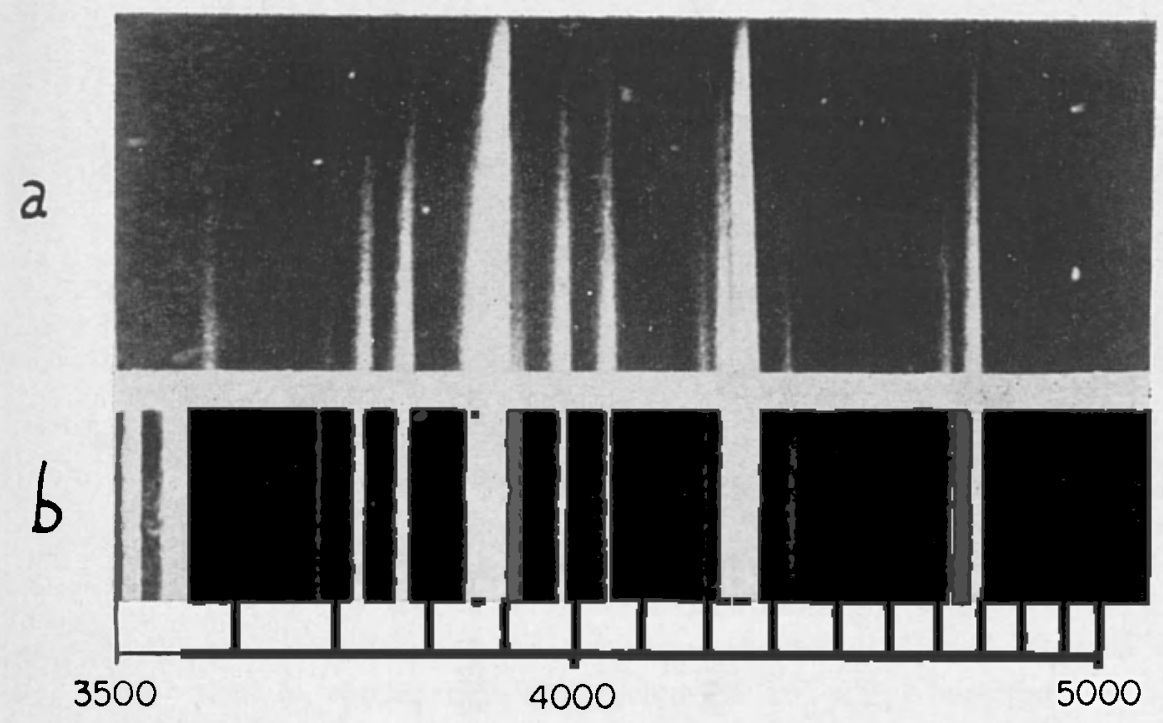

\section{$230 \mathrm{KeV}$ PROTONS}

\section{AURORA}

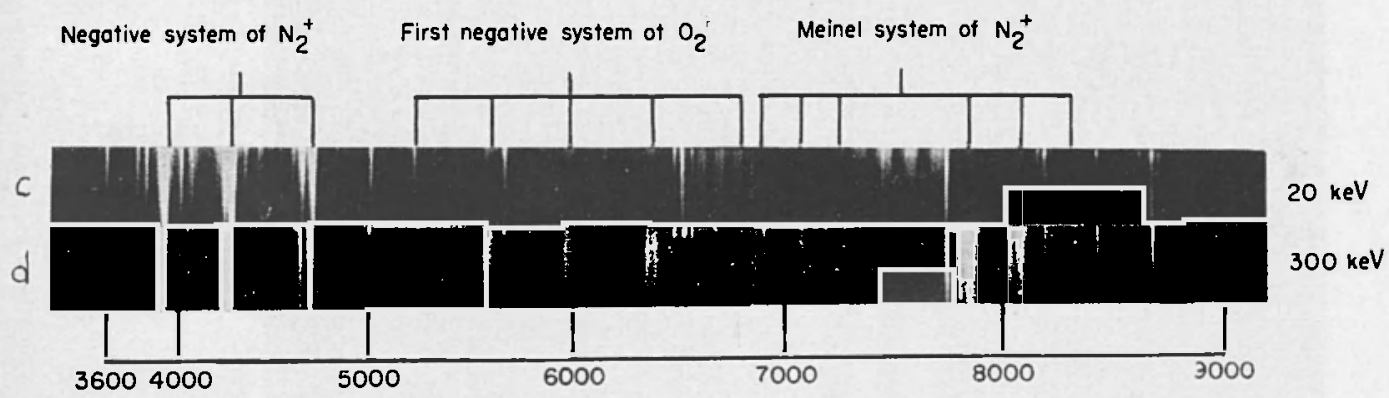

$a, b$ spettro ottenuto per eccitazione con protoni di $230 \mathrm{keV}$ e spettro aurorale di confronto; $c, d$ spettri ottenuti per eccitazione con protoni di 20 e $300 \mathrm{keV}$ rispettivamente: si notano sensibili differenze di intensità del gruppo di Meinel (per cortesia del dr. C. Y. Fan, Chicago). 

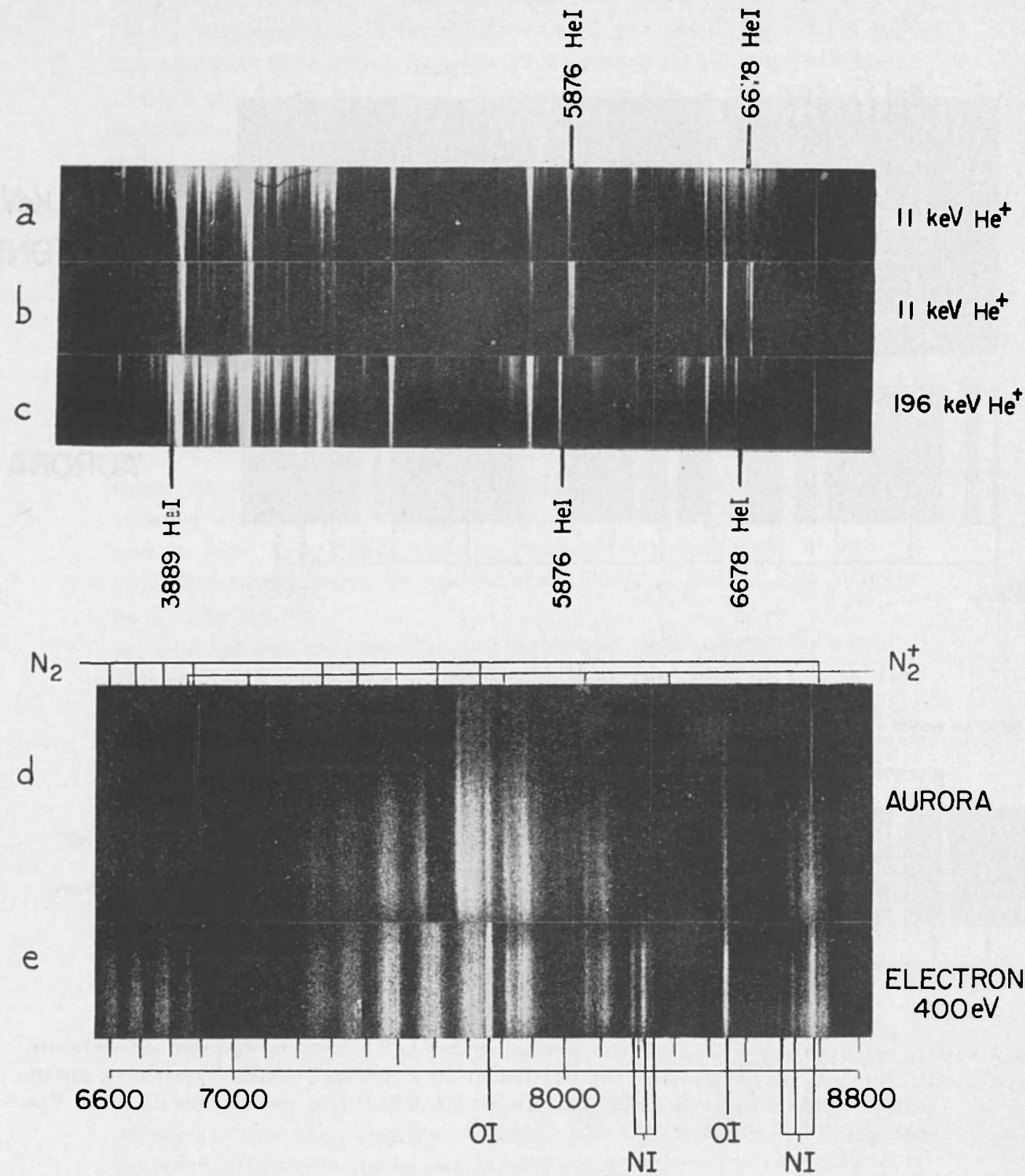

$a, b$, c spettri ottenuti per eccitazione con ioni $H e^{+}$di energia 11 e $196 \mathrm{keV}$ : lo spettro $b$, ottenuto a pressione di circa $10^{-4} \mathrm{~mm} \mathrm{Hg}$, mostra le righe stazionarie dell'HeI e le corrispondenti righe spostate per effetto Doppler; gli spettri $a$, $c$, invece, ottenuti a pressione di circa $10^{-3} m_{m} m_{\mathrm{Hg}}$, mostrano, per le divese condizioni di eccitazione, le sole righe spostate per effetto Doppler; $d$, e spettro ottenuto per eccitazione con elettroni di energia $400 \mathrm{eV}$ e spettro aurorale di confronto (per cortesia dei dr. C. Y. Fan, J. W. Chamberlain e A. B. Meinel dello Yerkes Observatory, Chicago). 
del fascio non sarebbe influenzato dal campo magnetico terrestre, la qual cosa non appare plausibile.

Si potrebbe allora supporre che l'eccitazione delle bande di Meinel sia dovuta a protoni di alta energia, in quanto alle alte energie il comportamento di qualunque tipo di particella, a parità di velocità, tende ad essere il medesimo (v. Massey e Burhop $\left({ }^{46}\right)$, cap. VIII paragrafo 6.1 ); in effetti, un protone di velocità pari a quella di un elettrone di $3 \mathrm{keV}$ ha una energia di circa $6 \mathrm{MeV}$. Ma protoni di tale energia dovrebbero scendere a quote assai basse nell'atmosfera, mentre è ben noto che aurore assai basse costituiscono l'eccezione e non la regola.

Scartata l'ipotesi dei protoni, come causa di eccitazione delle bande di Meinel, rimane come terza, più plausibile possibilità, quella che gli elettroni di bassa energia siano secondari e che essi siano accelerati da campi elettrici locali.

Il meccanismo che può dar luogo a tali campi elettrici può essere di varia natura: nel fascio incidente di particelle, protoni ed elettroni, essendo, a parità di velocità, i protoni più penetranti degli elettroni, può determinarsi un sopravanzamento dei primi rispetto ai secondi con la conseguente formazione di un campo elettrico radiale uscente dalla Terra che accelera verso il basso gli elettroni. Fan trova che per un flusso incidente di $10^{6}$ particelle $\cdot \mathrm{cm}^{-2} \cdot \mathrm{sec}^{-1}$ alla velocità $10^{9} \mathrm{~cm} / \mathrm{sec}$ si avrebbe una separazione tra i due tipi di particelle dell'ordine di qualche decina di $\mathrm{km}$, che appare troppo piccola per rendere conto della lunghezza normalmente assai maggiore dei raggi aurorali. La difficoltà potrebbe essere superata assumendo che già prima della penetrazione nella atmosfera sia in atto una separazione dei protoni e degli elettroni per azione del campo magnetico terrestre.

2.4.5. La eccitazione della luminosità aurorale. - Abbiamo già osservato come le diverse caratteristiche spettroscopiche degli archi e delle forme raggiate suggeriscono che fondamentalmente diversi debbano essere i processi che danno rispettivamente origine agli uni e alle altre. Negli archi si può ritenere accertata la presenza di protoni incidenti dall'esterno sulla atmosfera, che sembrano invece sistematicamente assenti nelle forme raggiate. Se si pensasse che queste ultime possano essere dovute a elettroni primari, alle ragioni contrarie, già esposte in 2.4.4.3, si aggiunge il fatto che gli andamenti calcolati della luminosità non corrispondono soddisfacentemente al caso di intensità luminose sensibilmente costanti al variare della quota (per densità di materia variabili in un rapporto da 1 a 100 e oltre, contro un corri- 
spondente rapporto da 1 a 10 per gli archi), come in effetti si riscontra nelle forme raggiate.

2.4.5.1. La teoria di Chamberlain. - L'autore calcola ( $\left.{ }^{78}\right)$ l'andamento della luminosità della riga $H_{\alpha}$ con la quota nell'ipotesi che essa sia emessa da atomi di idrogeno formati

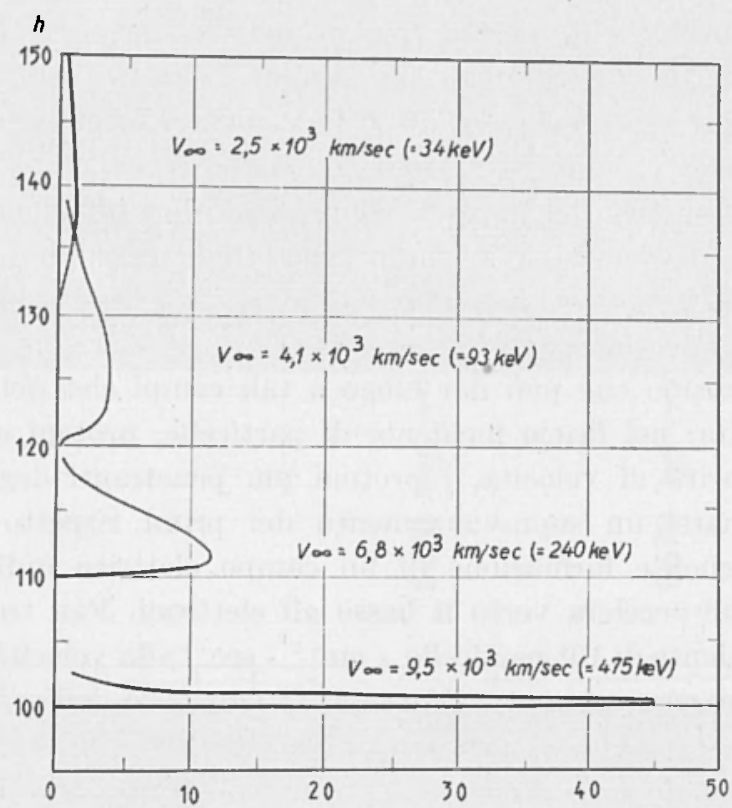

Fig. 2.17. - Altezza $h$ dal suolo in $\mathrm{km}$; sulle ascisse il numero di transizioni $H_{\alpha}$, in unità di $10^{-5} \mathrm{~cm}^{-3} \cdot \mathrm{sec}^{-1}$, prodotto da un flusso unitario di protoni. Sono indicate le velocità di incidenza "ळ e le energie corrispondenti (secondo Chamberlain).

dei protoni del fascio incidente; egli considera un fascio rettilineo di protoni incidenti nella direzione dello zenith e assume che ogni protone, attraverso ripetuti processi di cattura e riionizzazione, produca circa 50 quanti $H_{\alpha}$, ciò che corrisponde, per un arco aurorale di moderata intensità a un flusso di $6 \cdot 10^{7}$ protoni $\cdot \mathrm{cm}^{-2} \cdot \mathrm{sec}^{-1}$. La estensione verticale delle curve di luminosità decresce enormemente per velocità crescenti dei protoni monocromatici (fig. 2.17); Chamberlain determina anche il cosidetto "decremento di Balmer", rapporto tra le intensità delle tre righe $H_{\alpha}, H_{\beta}, H_{\gamma}$, che risulta essere $H_{\alpha}: H_{\beta}: H_{\gamma}=3,34: 1,00: 0,33$. A parità di quota di penetrazione, le curve di luminosità di Chamberlain risultano notevolmente più strette di quelle calcolate da Bates e Grif- 
fing $\left(^{45}\right)$ per la luminosità totale originata dalla penetrazione di protoni nell'aria (fig. 2.18). Occorre tuttavia notare che sia gli effetti della diffusione, sia la non monocromaticità dello spettro di velocità delle particelle,

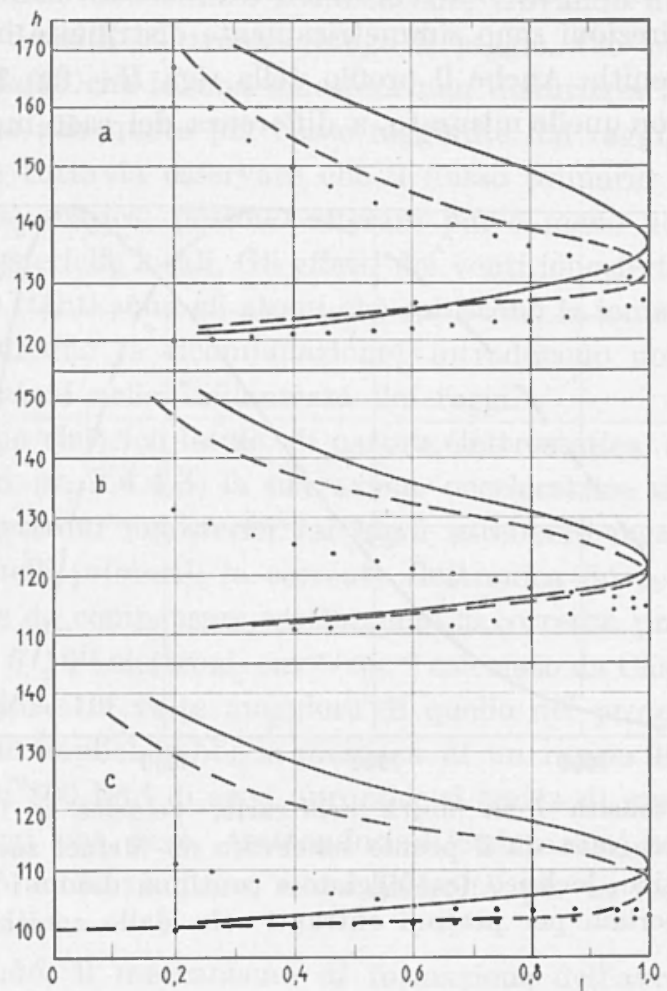

Fig. 2.18. - Altezza $h$ dal suolo in $\mathrm{km}$; luminosità $L$ in unità arbitrarie. Le linee continue danno gli andamenti sperimentali ottenuti da Harang per gli archi; quelle tratteggiate e quelle punteggiate danno gli andamenti calcolati da Chamberlain e da Bates e Griffing, rispettivamente. Le tre serie di curve $a, b$, $c$, si riferiscono ad archi il cui bordo inferiore raggiunge 120,110 , e $100 \mathrm{~km}$ dal suolo.

come anche la loro non monodirezionalità possono in realtà determinare un più o meno notevole aumento della estensione verticale della luminosità. Dal punto di vista sperimentale, si può aggiungere che Meinel ( $\left.{ }^{77}\right)$ ha effettivamente riscontrato per la luminosità $H_{a}$ una estensione verticale assai minore di quella osservata per altri tipi di emissione aurorale. 
In un successivo raffinamento della sua teoria, Chamberlain ha ottenuto curve di luminosità in discreto accordo (fig. 2.18) con quelle osservate nelle aurore, assumendo un fascio di protoni non più provenienti da una unica direzione (quella dello zenith), ma costituenti un fascio le cui direzioni sono simmetricamente distribuite intorno alla direzione dello zenith. Anche il profilo della riga $H_{\alpha}$ (fig. 2.19) risulta in buon accordo con quello misurato, a differenza del caso monodirezionale.

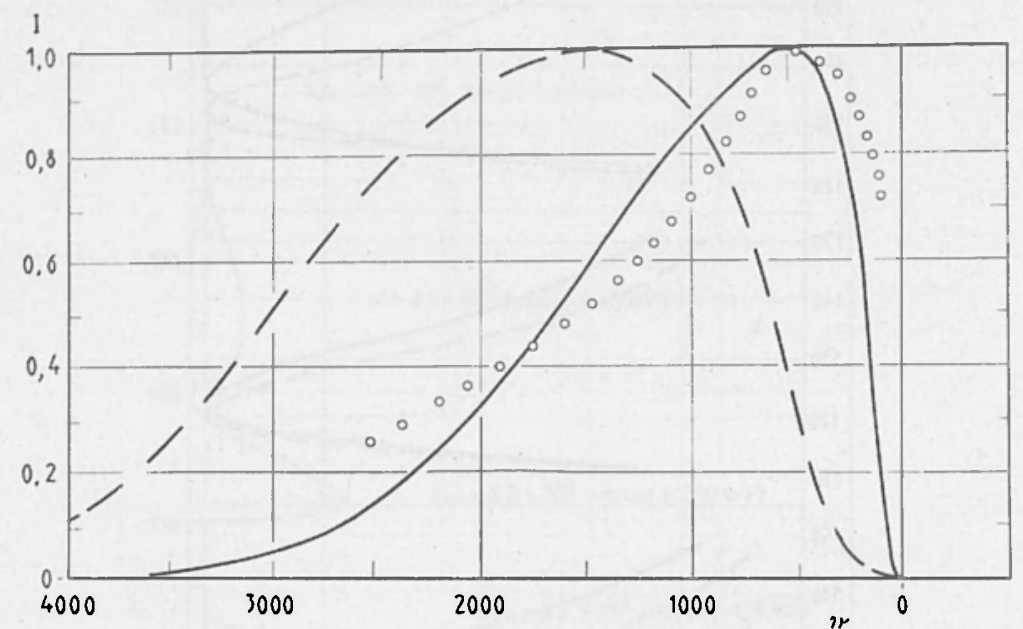

Fig. 2.19. - Intensità $I$ in unità arbitrarie, velocità $v$ in $\mathrm{km} / \mathrm{sec}$. La linea punteggiata dà il profilo osservato da Meinel secondo lo zenith geomagnetico; le linee tratteggiate e continue danno i profili calcolati da Chamberlain per protoni entranti solo dallo zenith o da tutte le direzioni.

Quanto ai raggi, Chamberlain $\left({ }^{78}\right)$ studia la ipotesi che la eccitazione della luminosità sia dovuta agli elettroni liberi presenti nella ionosfera, accelerati da campi elettrici "locali " dell'ordine dei $10^{-5}$ volt $/ \mathrm{cm}$ nella alta atmosfera. L'idea di un campo elettrico, come causa di accelerazione delle particelle aurorali, non è nuova in quanto già considerata da Alfven (v. cap. 6) e da altri $(7,25,74,79,80)$; la ipotesi di un campo elettrico locale lentamente decrescente al variare della quota potrebbe, sotto opportune condizioni, rendere conto della quasi costante luminosità dei raggi: infatti questa tenderebbe a diminuire al crescere della quota per la diminuzione della densità di materia, ma questa diminuzione potrebbe essere compensata dall'aumento del cammino libero medio e quindi della energia media acquisita dagli elettroni, un maggior numero dei quali raggiungerebbe la energia minima sufficiente a eccitare la luminosità. 
Seguendo tali linee, e assumendo una luminosità costante e una densità elettronica di $10^{8} \mathrm{~cm}^{-3}$ alla base di un raggio, corrispondente a un flusso costante di $6 \cdot 10^{12}$ elettroni $\cdot \mathrm{cm}^{-2} \cdot \mathrm{sec}^{-1}$, Chamberlain ha costruito un modello abbastanza soddisfacente trovando una lenta diminuzione della densità elettronica lungo il raggio. Ciò può apparire in contrasto col fatto che in una ionosfera non disturbata la densità elettronica, almeno alle quote più basse raggiunte dai raggi, cresce con la quota; occorre tuttavia osservare che il flusso primario di protoni, oltrechè la stessa scarica, possono alterare più o meno notęvolmente le condizionj ionosferiche locali. Gli effetti dei venti ionosferici e l'equilibrio di ionizzazione (tanti sono gli atomi che subiscono la ionizzazione e tanti quelli che subiscono la ricombinazione) introducono poi una serie di piccole fluttuazioni nella brillantezza dei raggi.

Se il campo elettrico locale, di natura elettrostatica, è prodotto dai protoni primari (v. 2.4.4.3) la sua azione acceleratrice si eserciterebbe su tutti gli elettroni ionosferici (ai quali sarebbero mescolati indistinguibilmente quelli primari); la corrente elettronica dovrebbe essere naturalmente tale da compensare esattamente la corrente protonica primaria. Il flusso di $6 \cdot 10^{12}$ elettroni $\cdot \mathrm{cm}^{-2} \cdot \mathrm{sec}^{-1}$ calcolato da Chamberlain per $\mathrm{i}$ raggi risulta circa $10^{5}$ volte maggiore di quello dei protoni negli archi (v. 2.4.4.2). Ciò implicherebbe la presenza di un raggio di $100 \mathrm{~m}$ di diametro per ogni $800 \mathrm{~km}^{2}$ di arco aurorale; si tratta di una troppo bassa densità di raggi che però, trattandosi di valutazioni necessariamente grossolane, può anche risultare, senza difficoltà per la teoria, $10^{2} \div 10^{3}$ maggiore.

Riassumendo, il meccanismo di formazione dell'aurora sarebbe il seguente: inizialmente le densità di corrente protonica ed elettronica (primarie) sarebbero dello stesso ordine di grandezza. Gli elettroni, per la loro bassa energia, non potrebbero eccitare alcuna luminosità; i protoni, invece, avendo assai più elevata energia, produrrebbero intensa ionizzazione, mentre una corrente elettronica richiamata dal campo elettrostatico dei protoni potrebbe seguire, ben concentrata, solo certi percorsi di minor resistenza elettrica dando origine ai raggi. Se questa situazione si stabilisce bruscamente si ha il rapido passaggio dall'arco alla forma raggiata, come spesso si osserva.

Il fatto che talvolta si osservino dei raggi senza alcuna apparente relazione con archi, non costituisce difficoltà in quanto, essendo assai alta la conducibilità della ionosfera, potrebbe anche aversi un afflusso di elettroni (cioè presenza di raggi) in zone anche lontane dalla zona in cui si concentra principalmente il flusso di protoni; d'altra parte, si 
potrebbe anche trattare di un flusso di protoni molto poco intenso distribuito su una area assai maggiore dell'usuale, così da non provocare apprezzabile luminosità del tipo degli archi, pur determinando ugualmente un campo elettrico.

2.4.5.2. La teoria di Lebedinsky. - L'autore ( $\left.{ }^{81}\right)$ assume un sistema di correnti che dai limiti dell'atmosfera fino alla quota dello strato $\mathbf{E}$ segue le linee di forza del campo magnetico terrestre, dando origine a raggi e drappeggi; nello strato $\mathrm{E}$, poi, le correnti scorrono orizzontalmente lungo i paralleli magnetici, dando origine in tal modo agli archi. Il passaggio dalla prima condizione alla seconda avviene a quota intorno ai $130 \mathrm{~km}$ dal suolo, al disotto della quale il cammino libero medio degli ioni e degli elettroni risulta determinato dalle collisioni con molecole neutre dell'atmosfera.

Lebedinsky confronta la energia elettrica associata alla corrente negli archi e nei raggi (che vanno pensati come conduttori in serie, percorsi da una stessa intensità di corrente dell'ordine dei $10^{4}$ ampere) e la energia luminosa emessa dalle particelle eccitate; trova che il rapporto energia luminosa/energia elettrica è prossimo all'unità per i raggi mentre è di circa 0,001 per gli archi; tale differenza viene attribuita al fatto che nei raggi l'eccitazione ha alto rendimento, in quanto provocata da urti elettronici, mentre negli archi essa deriva da collisioni ioniche di bassa efficienza.

\subsection{Fenomeni aurorali e perturbazioni geomagnetiche}

2.5.1. - Che le aurore e le tempeste magnetiche siano due fenomeni in stretta relazione l'uno con l'altro è noto già da lungo tempo: le maggiori tempeste magnetiche sono infatti sempre accompagnate da intense aurore, che spesso, in tali occasioni, appaiono anche a latitudini assai inferiori a quelle usuali.

Abbiamo già visto in $\mathbf{1 . 3 . 3}$ che la traccia dell'elettrogetto responsabile della forte attività magnetica delle alte latitudini coincide piuttosto bene con la curva di massima frequenza aurorale.

Una ulteriore prova della stretta interdipendenza tra aurore e perturbazioni geomagnetiche è data dalla fig. 2.20, nella quale sono tracciate le linee di uguale escursione media della $S_{D}$ per i giorni perturbati internazionali dell'Anno Polare 1932-33, nell'emisfero nord $\left(^{8}\right)$. Un confronto con la fig. 2.2, che rappresenta le isocasme di Vestine, permette di rilevare la generale somiglianza delle due rappresentazioni e la appros- 


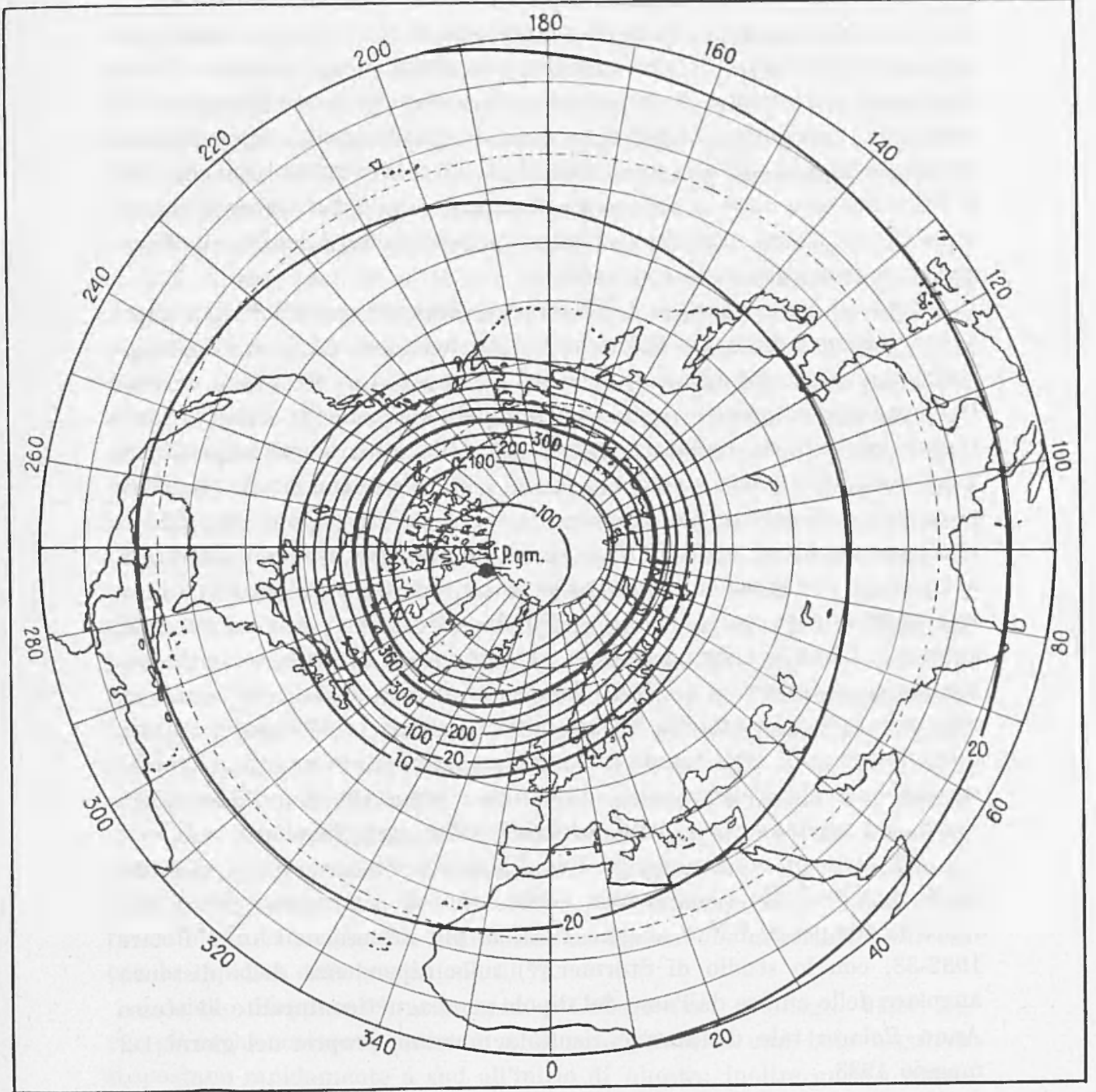

Fig. 2.20. - Linee di uguale escursione media della $S_{D}$. P. gm. polo geomagnetico nord (secondo Vestine). 
simativa coincidenza della curva di massima frequenza aurorale con quella di massima intensità delle perturbazioni geomagnetiche.

La stretta connessione tra i due fenomeni rende comprensibili le periodicità della frequenza aurorale di periodo undici anni, sei mesi (con massimi equinoziali) e 27 giorni, se tali periodicità potranno essere definitivamente confermate. A priori potremmo dire che le periodicità citate sono una necessaria conseguenza della correlazione tra perturbazioni geomagnetiche e aurore, e a latitudini alquanto inferiori ai $65-70^{\circ}$, a latitudini cioè dove la frequenza di apparizione delle aurore è ancora sensibile ma molto inferiore al 100\%, tali periodicità dovrebbero effettivamente essere messe in evidenza.

Come si è accennato in 1.3.3, appare piuttosto ragionevole pensare a una relazione fisica tra l'elettrogetto e i fenomeni aurorali. Tale supposizione appare giustificata anche dal fatto che durante le più intense tempeste magnetiche si verifica un notevole spostamento verso le latitudini inferiori sia dell'elettrogetto, sia delle apparizioni aurorali. In 1.3.4 sono stati citati alcuni casi in cui è stata determinata la posizione geografica dell'elettrogetto. Harang $\left(^{82}\right)$ trova per la regione scandinava uno spostamento di circa $400 \mathrm{~km}$ verso sud tra i giorni meno perturbati e quelli di perturbazioni più intense durante l'Anno Polare 1932-33, e tale spostamento porta l'elettrogetto da nord a sud di Tromsö (lat. geomagn. $67^{\circ} \mathrm{N}$ ); l'aurora visuale, che nei giorni di bassa attività geomagnetica compare a nord di Tromsö piuttosto bassa sull'orizzonte, all'aumentare della attività viene osservata ad altezze sempre maggiori, e in occasione di forti tempeste raggiunge e supera lo zenith. L'Autore ne conclude che l'elettrogetto e l'aurora si spostano simultaneamente verso sud all'aumentare della intensità della perturbazione.

Ad analoga conclusione si può giungere confrontando i risultati di Nagata ( ${ }^{83}$ ) sullo spostamento verso sud dell'elettrogetto polare durante la tempesta del $1^{\circ}$ maggio 1933, la più intensa dell'Anno Polare 1932-33, con lo studio di Störmer $\left.{ }^{(84}\right)$ sulla dipendenza della distanza angolare delle aurore dall'asse del dipolo geomagnetico durante lo stesso Anno Polare: tale distanza è risultata massima proprio nei giorni 1-2 maggio 1933.

2.5.2. - Stabilita una connessione generale tra le perturbazioni geomagnetiche prodotte dalle correnti elettriche polari e l'aurora, è di fondamentale interesse cercare delle relazioni più particolari tra l'attività geomagnetica e le varie caratteristiche aurorali, quali la luminosità, la forma, le trasformazioni da una forma all'altra, i moti, ecc. Sebbene 
questo studio sia ancora agli inizi, tuttavia sono stati già raggiunti alcuni risultati interessanti.

Già Harang ${ }^{82}$ ) aveva notato, misurando la luminosità integrata dalle aurore visibili a Tromso mediante apposita registrazione fotografi$\mathrm{ca}$, che tale luminosità tende ad aumentare al diminuire della latitudine del sistema di correnti generanti le perturbazioni geomagnetiche; è inoltre evidente, negli esempi che l'A. presenta, un forte e implov viso aumentare di luminosità all'apparire di una larga e brusca baia negativa nella componente $H$ del campo magnetico terrestre.

Dalle osservazioni di Meek a Saskatoon ( ${ }^{85}$ ) (lat. geomagn. 60,6 $\mathrm{N}$ ) si può trarre qualche ulteriore conclusione sulle caratteristiche della luminosità delle aurore e della loro altezza sull'orizzonte durante una perturbazione geomagnetica polare (v. 1.3.5). All'inizio di una baia positiva in $H$, che rappresenta in generale la prima fase della perturbazione, o parte di essa, appare in genere a nord di Saskatoon un arco piuttosto brillante, ma molto basso sull'orizzonte (la sua altezza non arriva di solito a superare i $1^{\circ}$ ); quando $H$ torna verso $l l$ valore normale e la baia tende a scomparire, la luminosità si attenua e la forma dell'aurora diviene più diffusa. Durante la seconda fase della perturbazione che, come è noto, è generalmente la più intensa ed è costituita da una o più baie negative, anche l'aurora presenta la sua fase più intensa; nel periodo antecedente la baia negativa è generalmente visibile a nord di Saskatoon una debole luminosità aurorale, residuo dell'arco concomitante alla precedente baia positiva; all'inizio della baia compaiono, ad una altezza maggiore sull'orizzonte, bande e drappeggi, e la luminosità aumenta. Secondo Meek l'altezza dell'aurora sull'orizzonte è in stretta relazione con l'intensità della perturbazione magnetica: una baia negativa di 100 gamma in $H$ corrisponde, a Saskatoon, a un'altezza massima di $60^{\circ}$, mentre una caduta in $H$ di 200 gamma corrisponde ad aurora visibile allo zenith. La luminosità invece sarebbe in relazione diretta con la rapidità della variazione di $H$.

Anche Heppner $\left(^{86}\right)$, riscontra a College (lat. geomagn. $64,5^{\circ} \mathrm{N}$ ), che gli archi omogenei presenti verso nord prima di una baia negativa si spostano rapidamente a sud all'inizio di questa; inoltre questo movimento è accompagnato dalla formazione di strutture raggiate a nord dell'arco. Con l'intensificarsi della perturbazione geomagnetica anche l'arco si spezza in forme raggiate attive che si estendono anche oltre lo zenith, seguite da aurora diffusa e pulsante; il graduale ritorno di $H$ verso il suo valore normale è ancora accompagnato da strutture diffuse e pulsanti. 
Le osservazioni di Heppner appaiono confermate da quelle di Fan ( $\left.{ }^{87}\right)$ eseguite all'Osservatorio di Yerkes, a latitudine geomagnetica notevolmente inferiore ( $\left.53^{\circ} \mathrm{N}\right)$. Fan trova infatti che la componente $H$ aumenta progressivamente prima della apparizione di un arco omogeneo, e che l'inizio di una baia negativa coincide con la trasformazione dell'arco omogeneo in struttura raggiata.

Tutte queste osservazioni sono assai importanti ai fini di una teoria sulle aurore e sulle perturbazioni geomagnetiche polari. Alcuni Autori, e in particolar modo Fukushima $\left(^{(88)}\right)$, pensano che queste ultime siano dovute a un effetto "dinamo ", simile a quello cui è dovuta la $S_{q}$, con la differenza che la ionizzazione dell'alta atmosfera (della zona polare) avviene questa volta sotto l'azione di fasci di particelle incidenti alle alte latitudini. Oltre alla ionizzazione, tali fasci produrrebbero anche le aurore. In tal caso, tuttavia, le forze elettromotrici responsabili delle correnti ionosferiche sarebbero sempre presenti, a causa dei moti regolari dell'alta atmosfera, e dovrebbero variare indipendentemente dai fasci di particelle e quindi dalle aurore. Ma le osservazioni sopra descritte, in particolar modo quelle di Heppner, sembrano dimostrare invece una stretta correlazione tra aurore e forze elettromotrici, in quanto le forme aurorali cambiano quando anche le forze elettromotrici cambiano.

Alla luce di queste considerazioni appare estremamente interessante il fatto, constatato da Fan e Schulte $\left(^{58}\right)$ e messo ulteriormente in evidenza da Fan ${ }^{\left({ }^{87}\right)}$, che al momento della trasformazione di un arco omogeneo in struttura raggiata, cioè all'inizio di una baia negativa, si ha anche- una trasformazione dello spettro aurorale, almeno per quanto riguarda le righe dell'idrogeno: mentre queste sono spesso presenti nello spettro dell'arco, la loro intensità diminuisce rapidamente fino ad annullarsi quando si formano le strutture raggiate (v, anche 2.4.4.1).

\section{RINGRAZIAMENTI}

Vogliamo esprimere la nostra gratitudine al prof. C. T. Elvey e al dr. V. P. Hessler del Geophysical Institute University of Alaska, per le fotografie di aurore e di spettri aurorali; ai dr. J. W. Chamberlain, C. Y. Fan e D. H. Schulte dello Yerkes Observatory dell'Università di Chicago per gli spettri aurorali e di laboratorio.

Ringraziamo anche lo Sveriges Meteorologiska och Hydrologiska Institut di Stoccolma e la Antartic Division del Department of External Affaires di Melbourne per avere messo a nostra disposizione i risultati delle loro osservazioni aurorali. 


\section{RIASSUNTO}

Si presenta la fenomenologia delle aurore polari, in particolare le loro forme visuali più tipiche, la loro distribuzione geografica e le variazioni tem. porali. Si discutono quindi le caratteristiche del loro spettro, in relazione al tipo di agente che può provocarle. Si esaminano le relazioni tra aurore e attività solare, nonchè tra aurore ed attività geomagnetica.

\section{ABSTRACT}

We consider the phenomenological aspect of polar aurorae, particularly their visual appearance, their grographical distribution and time variations. We discusss also the physical structure of auroral spectra, with particular regard to the effective causes of auroral phenomena. At last, we study the correlations of aurorae with solar and geomagnetic activity.

\section{BIBLIOGRAFIA}

(A) Störner C., The Polar Aurora, Oxford, Clarendon Press (1955).

(B) Harang L., The Aurorae, Londra, Chapman Hall Ltd (1951).

(C) Fritz II., Das Polarlicht, Leipzig, F. A. Brockhaus (1881).

(1) Photographic Atlas of Auroral Forms, International Geodetic and Geophysical Union, Oslo (1930).

(2) A nnals of I.G. F. 4, parte II, Londra, Pergamon Press.

(3) Loveli. A. C. B., Clegg J. A., Ellyett C. D., Nature 160, 372 (1947).

(1) Aspinall A., Hawkins G. S., Journ. Brit. Astr. Assoc. 60, 130 (1950).

(5) Harang L., Landmark B., Journ. Atm. Terr. Phys. 4, 322 (1954).

( ${ }^{8}$ Currie B. W., Forsyti P. A., Vawter F. E., Journ. Geoph. Res. 58, 179 (1953).

(7) Bowles K. I., Journ. Geoph. Res. 59, 553 (1954).

( $\left.{ }^{8}\right)$ Vestine E. H., Terr. Magn. 49, 77 (1944).

(9) Vestine E. H., SNydir E. J., Terr. MLagn. 50, 105 (1945).

(10) Little D. E., Shrum G. M., Trans. Roy. Soc. Can. 44, 51 (1950).

(11) Vegard L., Krogness O., Geofys. Publ. 1, n. 1 (1920).

(12) Störmer C., Geofys. Publ. 4, n. 7 (1926).

(13) Carlineim Gyllenskiold V., A urores Boreales, Stocolma (1887).

(is) Harang L., Terr. Magn. 50, 297 (1945).

(15) Jensen R. E., Currie B. W., Journ. Geoph. Res. 58, 201 (1953).

(19) Currie B. W., Terr. Magn. 39, 293 (1934).

(17) Fuller V. R., Bramhall E. H., Misc. Publ. Univ. Alaska, 3 (1937). 
(18) Alty T., Wilson F. J., Nature 133, 687 (1934).

(19) Unwin R. S., Gadsden M., Nature 180. 1469 (1957).

${ }^{(20)}$ Bullougit K., Kaiser T. R., Journ. Atm. Terr. Phys. 5, 189 (1954).

(21) STÖRMER C., Terr. Magn. 53, 251 (1948).

(22) EGED AL J., Nature 123, 642 (1929).

(23) EGEDAL J., Nature 124, 913 (1929).

(24) S'ö́rmer C., Geofys. Publ. 13, n. 7 (1942).

(25) Meinel A. B., Schulte D. H., Astroph. Journ. 117, 454 (1953).

$\left({ }^{26}\right)$ Bless R. C., Gartlein C. W., Kimball I). S., Astroph. Journ. 122, 205 (1955).

(27) Meinel A. B., Astroph. Journ. 122, 206 (1955).

$\left({ }^{28}\right)$ Kin J. S., Currie B. W., Can. Journ. Phys. 36, J60 (1958).

$\left({ }^{29}\right)$ Vegard I., Phil. Mlag. 23, 211 (1937).

${ }^{(30)}$ Fuller V. B., Terr. Magn. 40, 269 (1935).

(31) Sverdrup H. V., Res. Dept. Terr. Magn. Carnegie Inst. Washington, 6, n. 175 (1927).

(32) Meinel A. B., Negaard B. J., Chamberlain J. IV., Journ. Geoph. Res. 59, 407 (1954).

$\left({ }^{33}\right)$ Angot A., Les Aurores polaires, p. 125-6.

(4) Gerson N. C., Suppl. Nuovo Cim. 4, 1562 (1956).

$\left({ }^{35}\right)$ Tromнlт S., Katalog der in Norwegen bis Juni 1878 beobachteten Nordlichter, Kristiania (1902).

$\left({ }^{36}\right)$ BoLler W., Gerlands Beitrage zur Geophysik 5, 3 (1898).

${ }^{(37}$ ) Stetson II., Science 90, 482 (1939).

${ }^{38}$ ) Garner, New Zeal. Journ. of Science and Technology 33, n. 1 (1951).

${ }^{(39)}$ Tromholt S., Danish Meteor. Inst. Yearbook (1880).

${ }^{(40)}$ Schmidt A., Meteor. Zeitschrift 37, 163 (1920).

(41) ANGenileister G., Meteor. Zeitschrift 39, 20 (1922).

(42) II arang L., Geofys. Publ. 16, n. 6 (1945).

${ }^{43}$ ) Lenard P. D., Sitz. d. Heidelberger A\%. ad., n. 17 (1910) e n. 12 (1911).

(44) Vegard L., Plil. Mag. 42, 47 (1921).

$\left({ }^{45}\right)$ Bates D. R., Griffing G., Journ. Atm. Terr. Phys. 3, 212 (1953).

${ }^{(46)}$ Massey H. S. W., Buriop E. H. S., Electronic and Ionie Impact Phenomena, Oxford, Clarendon Press (1952).

(47) Vegard L., Geofys. Publ. 19, n. 9 (1956).

(48) Vegard L., Nature. 144. 1089 (1939).

(49) Vegard L., Terr. Magn. 45, 5 (1940).

${ }^{\left({ }^{50}\right)}$ Bernard R., Rep. Conf. of Gassiot Comm. Roy. Soc., Emission Spectra of the Night Sky and Aurora, p. 93, Londra, Phys. Soc. (1948).

(51) Meinel A. B., Astroph. Journ. 114, 431 (1951).

${ }^{(52)}$ Vegard L., Nature. 165, 1012 (1950).

${ }^{(53)}$ Vegard L., C. R. Acad. Sci. Paris 230, 1884 (1950).

$\left({ }^{54}\right)$ Gartlein C. W., Trans. Amer. Geoph. Union 31, 18 (1951).

${ }^{(55)}$ Meinel A. B., Phys. Rev. 80, 1096 (1950).

${ }^{(56)}$ Meinel A. B., Astroph. Journ. 113, 50 (1951).

${ }^{\left({ }^{57}\right)}$ Meinel A. B., Proceedings of the Conference on Auroral Physics, London, Ontario, July 1951, ed. N. C. Gerson.

${ }^{(58)}$ Daimlstrom C. E., Hunten D. M., Phys. Rev. 84, 378 (1951). 
$\left({ }^{59}\right)$ Fan C. Y., Schulte D. H., Astroph. Journ. 120, 563 (1954).

$\left({ }^{80}\right)$ Gartlein C. W., Memoires de la Societé Royale des Sciences de Liège 12, 195 (1952).

(11) VEgARD L., Nature 170, 536 (1952).

${ }^{62}$ Mott N. F., Massey H. S. W., The Theory of Atomic Collisions, Oxford, Clarendon Press, 1949, cap. XI.

(83) Richardson R. S., Trans. Amer. Geoph. Union 25, 558 (1944).

(64) Bruck A. H., Rutllant F., Monthly Not. Roy. Astr. Soc. 106. 130 (1946).

${ }^{\left({ }^{5}\right)}$ Gartlein C. W., Phys. Rev. 81, 463 (1951).

${ }^{\left({ }^{6}\right)}$ Galperin G. I., Planetary and Space Science 1, 57 (1959).

${ }^{(67)}$ Hunten D. M., Journ. Atm. Terr. Phys. 7, 14 (1955).

( $\left.{ }^{68}\right)$ Gartlein C. W., Sprague G., Journ. Geoph. Res. 62, 521 (1957).

$\left({ }^{89}\right)$ Meinel A. B., F AN C. Y., Astroph. Journ. 115, 330 (1952).

(70) Fan C. Y., Meinel A. B., Astroph. Journ. 118, 205 (1953).

(71) FAN C. Y., Astroph. Journ. 122, 350 (1955).

(72) Fan C. Y., Phys. Rev. 103, 1740 (1956).

(73) Krasner S., Phys. Rev. 99, 520 (1955).

(74) FAN C. Y., Astroph. Journ. 119, 294 (1954).

(75) Fan C. Y., Journ. Atm. Terr. Phys. Supplement 3, The Airglow and the Aurorae, p. 276, Londra, Pergamon Press (1956).

(76) Chamberlain J. W., Astroph. Journ. 120, 360 (1954).

(77) Meinel A. B., Memoires de la Societé Royale des Sciences de Liège 12, 203 (1952).

(78) Chamberlain J. W., Journ. Atm. Terr. Phys. Supplement 3, The Airglow and the Aurorae, p. 206, Londra, Pergamon Press (1956).

(79) WuLF O. R., Journ. Geoph. Res. 58, 553 (1953).

(80) Chamberlain J. W., Astroph. Journ. 122, 349 (1955).

(81) Lebedinsky A. J., Journ. Atm. Terr. Phys. Supplement 3, The Airglow and the Aurorae, p. 222, Londra, Pergamon Press (1956).

(82) Harang L., Terr. Magn. 51, 353 (1946).

(83) Nagata T., Journ. Geoph. Res. 55, 127 (1950).

(84) Stormer C., Geofys. Publ. 18, n. 7 (1953).

(85) МеЕк J. H., Journ. Geoph. Res. 58, 445 (1953) e 59, 87 (1954).

${ }^{(86)}$ Heppner J. P., Journ. Geoph. Res. 59, 329 (1954).

(87) F AN C. Y., Astroph. Journ. 128, 420 (1958).

(88) Funushima N., Polar geomagnetic storms and geomagnetic bays, Geoph. Inst., Tokyo Univ. (1953).

Direttore: Prof. ENRICo MEdI

Responsabile: Prof. Pietro CaloI

Tipografia Pio X, Via degli Etruschi, 7-9 - Roma-1-8.59 